\title{
Computer models of endo-epicardial dissociation of electrical activity and transmural conduction during atrial fibrillation
}

Citation for published version (APA):

Gharaviri, A. (2016). Computer models of endo-epicardial dissociation of electrical activity and transmural conduction during atrial fibrillation. [Doctoral Thesis, Maastricht University]. Maastricht University. https://doi.org/10.26481/dis.20160429ag

Document status and date:

Published: 01/01/2016

DOI:

10.26481/dis.20160429ag

Document Version:

Publisher's PDF, also known as Version of record

Please check the document version of this publication:

- A submitted manuscript is the version of the article upon submission and before peer-review. There can be important differences between the submitted version and the official published version of record.

People interested in the research are advised to contact the author for the final version of the publication, or visit the DOI to the publisher's website.

- The final author version and the galley proof are versions of the publication after peer review.

- The final published version features the final layout of the paper including the volume, issue and page numbers.

Link to publication

\footnotetext{
General rights rights.

- You may freely distribute the URL identifying the publication in the public portal. please follow below link for the End User Agreement:

www.umlib.nl/taverne-license

Take down policy

If you believe that this document breaches copyright please contact us at:

repository@maastrichtuniversity.nl

providing details and we will investigate your claim.
}

Copyright and moral rights for the publications made accessible in the public portal are retained by the authors and/or other copyright owners and it is a condition of accessing publications that users recognise and abide by the legal requirements associated with these

- Users may download and print one copy of any publication from the public portal for the purpose of private study or research.

- You may not further distribute the material or use it for any profit-making activity or commercial gain

If the publication is distributed under the terms of Article 25fa of the Dutch Copyright Act, indicated by the "Taverne" license above, 


\section{Computer Models of Endo-Epicardial}

Dissociation of Electrical Activity and Transmural Conduction During Atrial Fibrillation

Ali Gharaviri 
Copyright(C Ali Gharaviri, Maastricht 2016 Cover design: Paniz Afroughi

Printed by: Gilderprint - The Netherlands ISBN: $\quad$ 978-94-6233-280-5 


\title{
Computer Models of Endo-Epicardial Dissociation of Electrical Activity and Transmural Conduction During Atrial Fibrillation
}

\author{
Dissertation \\ To obta in the degree of Doctor at Maastricht University, \\ on the authority of the Rector Magnific us, Prof. dr. LL.G.Soete, \\ in accordance with the decision of the board of Deans, \\ to be defended in public \\ on Friday $29^{\text {th }}$ April 2016, at 12:00 o' clock \\ by
} Ali Gha raviri 


\section{Supervisor:}

Prof. Dr. U. Schotten

\section{Co-supervisor:}

Dr. S.Verheule

\section{Assessment Committee:}

Prof. Dr. T. Delhaas (chairman)

Prof. Dr. F.W. Prinzen

Prof. Dr. rer. nat. O. Dössel (Karlsruhe Institute of Technology)

Dr. N. Virag (Medtronic Inc.) 


\section{Table of contents}

$\begin{array}{lll}\text { Chapter } 1 & \text { General Introduction } & 7\end{array}$

Chapter 2 A Computer Model of Endo-Epicardial Electrical Dissociation and Transmural Conduction during Atrial Fibrillation

Chapter 3 How Disruption of Endo-epicardial Electrical

Connections Enhances Endo-epicardial

Conductionbduring Atrial Fibrillation

Chapter 4 Loss of continuity in the thin epicardial layer due to endomysial fibrosis increases the complexity of atrial fibrillatory conduction

Chapter 5 Effect of $\mathrm{Na}+$ channel blockade on the threedimensional substrate of atrial fibrillation in a model of endo-epicardial dissociation and transmural conduction

Chapter 6 A 3D model of endo-epicardial dissociation and transmural conduction during atrial fibrillation

Chapter 7 General discussion 


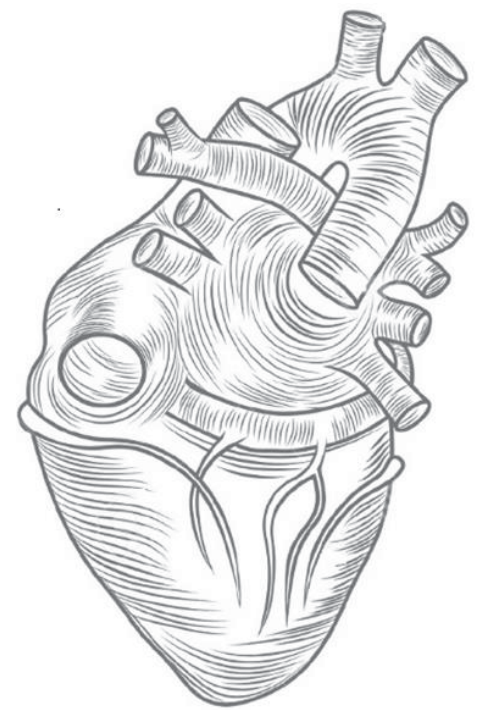




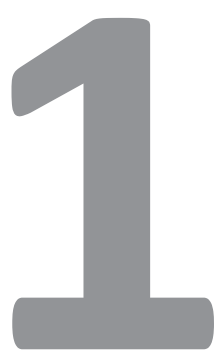

General introduction

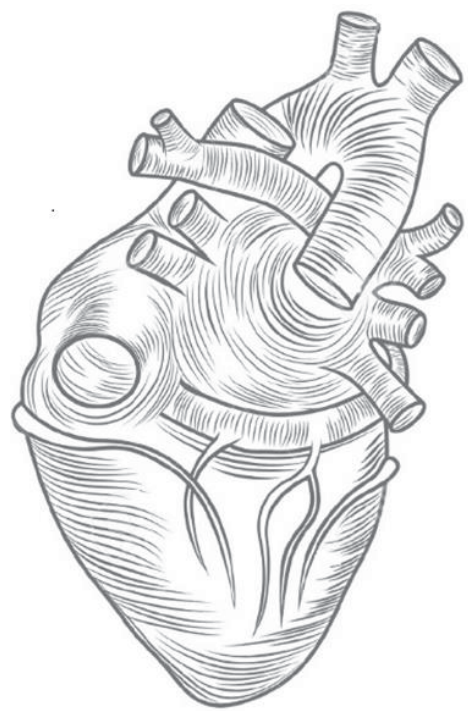




\section{General introduction:}

Atrial fibrillation (AF) is the most common cardiac arrhythmia. ${ }^{1}$ The prevalence of atrial fibrillation increases with age from 0.5 percent of people under the age of 60 and to more than 10 percent of people over the age of $80 .^{2}$ Atrial fibrillation, in the majority of patients, progresses from short episodes to longer episodes of AF (paroxysmal AF) and thereafter to sustained forms of $A F$ (persistent AF). ${ }^{3}$

The progression of atrial fibrillation is caused by two different kinds of remodeling, called electrical and structural. ${ }^{4}$ Electrical remodeling is a relatively fast process (1-2 days) that includes shortening of the action potential and the effective refractory period. However structural remodeling develops much more slowly (months-years) and is considered as the main mechanism leading to AF stability. The later process includes alteration in connexin expression ${ }^{5}$, myocyte hypertrophy ${ }^{6}$, and atrial fibrosis. ${ }^{7}$

AF - in its paroxysmal stage - is often initiated by ectopic activity or rapid reentrant circuits, often located in the muscular sleeves of the pulmonary veins. ${ }^{8}$ Sustained AF causes progressive structural damage of the atrial wall. Using high-density epicardial mapping contact mapping, both in experimental and clinical studies, our group has shown that progression of structural remodeling and AF stability go hand in hand with an gradual increase in complexity of the AF conduction pattern. ${ }^{9-13}$ The increase in conduction pattern includes the increase in the presence of the number of simultaneous fibrillation waves and the occurrence of conduction blocks, which leads to a more dissociated pattern of fibrillatory conduction (see figure 1A). In addition to the decrease in the size of wavefronts and increase in number of simultaneous existing waves, incidence of breakthroughs also increases (see figure 1B). ${ }^{14}$ Breakthroughs are waves, which appear within the mapping area and cannot be explained by propagation of existing waves within the mapping area. In principle the presence of these waves can be explained either by ectopic focal discharges or by transmural conduction from the opposing layer of the atrium. ${ }^{14}$ 
A fibrillation wave can only propagate transmurally, from the endocardium to the epicardium or vice versa, if the opposing layer is excitable. ${ }^{14}$ This means that one layer needs to be activated and the other not. Therefore the presence of endo-epicardial electrical activity dissociation is absolutely essential for the transmural conductions. Endo-epicardial dissociation was first described in exvivo model by Schuessler et al. ${ }^{15}$ They performed a simultaneous endo-epicardial mapping in isolated canine right atrial in the presence of acetylcholine and found significant activation time differences between epicardial and endocardial layer. ${ }^{14}$ Eckstein et al. directly assessed the degree of endo-epicardial dissociation and the origin of the breakthroughs, using simultaneous endo-epicardial high-resolution direct contact mapping tool. ${ }^{10}$ They showed a significant increase in the level of endo-epicardial dissociation in goats in 6 months of AF compared to the goats in 3 weeks of AF and acute AF. ${ }^{10}$ They found that the increase in the level of endoepicardial dissociation was well correlated with the breakthroughs incidence. $^{14}$ This finding strongly supports the argument that breakthroughs are indicative for a 3-dimensional character of electrical propagation during $A F^{10,14}$ 


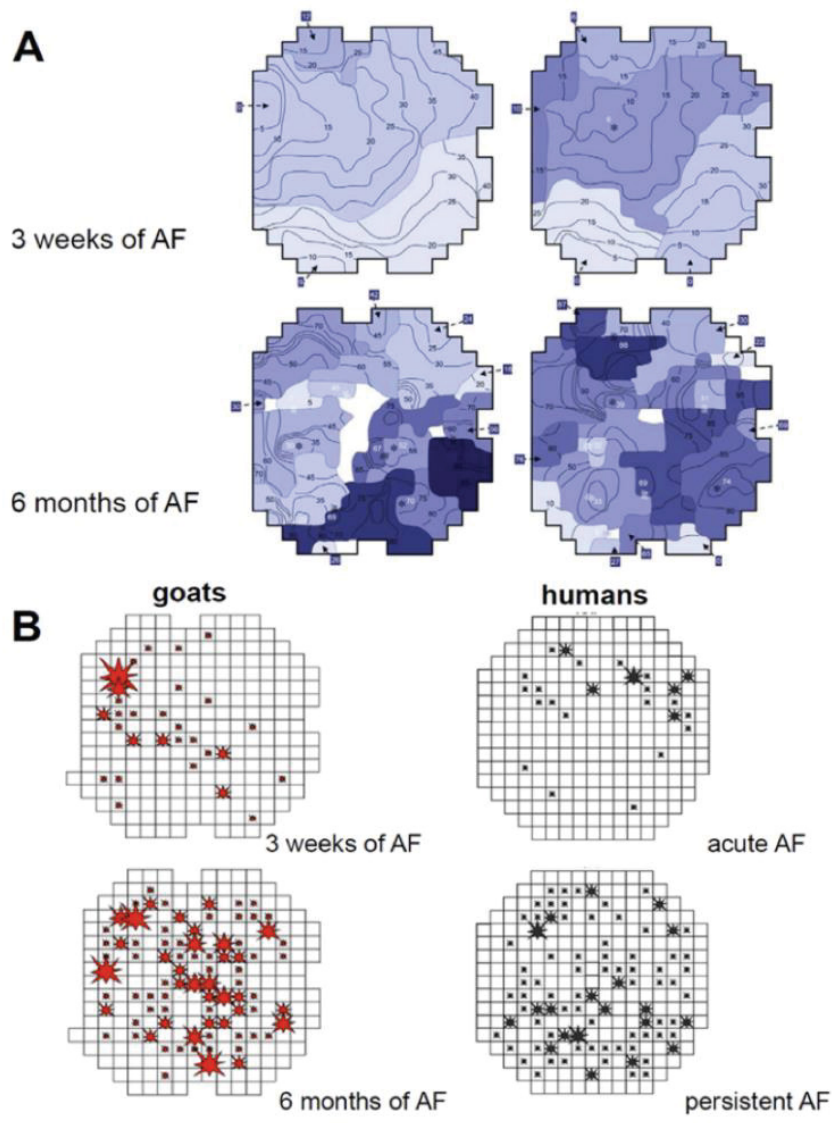

Figure 1. Increasing complexity of fibrillatory conduction during the development of an AF substrate. A) Increasing complexity of fibrillatory conduction during the development of an AF substrate. Two successive maps recorded during $A F$ in the $L A$ of goats after 3 weeks (upper row) and 6 months of AF (lower row). These time points correspond to stages in the disease process with complete electrical remodeling without and with significant structural remodeling. The wave maps of fibrillatory conduction show separate fibrillation waves in different shades of blue. Isochrones indicate conduction within the area of a particular wave. Epicardial breakthroughs are indicated with asterisks. After 6 months of AF, the fibrillation pattern was more complex, with a higher number of smaller fibrillation waves and an increased incidence of epicardial breakthroughs. B) Distribution of epicardial breakthroughs, the size of the stars indicates the number of breakthroughs observed at a particular electrode. In both goats and patients, the frequency of epicardial breakthroughs was higher in chronic AF, but the distribution was also less clustered. Adapted from refs (de Groot et al., 2010 and Verheule et al., 2014). ${ }^{13,14}$ 


\section{Computer models contribute to understanding the mechanisms of AF}

Numerous qualitative and quantitative computer models have been designed to simulate electrical propagation in the heart tissue.

Models developed so far to understand mechanisms underlying AF can be divided in two categories with respect to their geometrical properties. The first category includes surface models, which treat the atria as a two dimensional sheet or a two dimensional sheet folded into the shape of the atria (with two main cavities, atrial appendages, and inter atrial connections at the septal ring). ${ }^{16-18}$ The second category includes 3D volumetric models. These models include greater details in modeling the atria such as variations in wall thickness or local fiber orientation, and complex endocardial bundle networks. ${ }^{19-24}$ Although all the models mentioned above were useful to answer many important questions regarding the mechanisms contributing to AF perpetuation none of them simulated breakthroughs and endo-epicardial dissociation of electrical activity.

\section{Aim of this thesis}

The work presented here aims to contribute to our understanding of the 3-dimensional nature of the substrate for AF. Its ultimate goal is to better understand the mechanisms underlying atrial fibrillation and to design a novel framework for further investigation of endo-epicardial dissociation of electrical activity as a prerequisite for breakthroughs occurrence.

In chapter 2, a novel proof-of-principle dual layer model is introduced. This is the first model simulating dyssynchronous electrical activity in two separate layers - representing epicardium and endocardium - coupled by electrical connection points enabling transmural conduction and 'breakthrough' of fibrillation waves from one layer to the other. In this model we studied the effect of the existence of the transmural conduction on the stability of the 
fibrillatory process and the dynamic properties of fibrillation waves. In chapter 3 of this thesis the model introduced in the chapter 2 was used to study the effect of the progressive loss of endoepicardial electrical connections on the degree of endo-epicardial electrical activity dissociation, breakthrough rate, and AF stability. Finally, the findings in this chapter were compared with experimental findings. In chapter 4 , we have investigated the spatial distribution of fibrillation waves and structural alterations in the atrial free walls in both a goat model of AF and double layer model. Using the same model introduced in chapter 3, we identified the effect of isolated epicardial fibrosis on the complexity of conduction pattern and transmural propagation during atrial fibrillation. In chapter 5, the double layer model were used to address the question why a $\mathrm{Na}$-channel blockers, such as Flecainide, is effective, in terms of AF termination, in early stages of AF but gradually loses its efficacy in the later stages of the arrhythmia. In chapter 6 , the concept of chapter 2 and 3 were extended to a highly detailed anatomical model of human atrium. The model is applied to study the effect of epicardial fibrosis, what we found experimentally in chapter 4 , on atrial fibrillation conduction pattern complexity and epicardial breakthroughs. Finally in chapter 7, we discussed the introduced models, their applications, and possible improvements. 


\section{References:}

1. Nattel S. New ideas about atrial fibrillation 50 years on. Nature Jan 10 2002;415:219-226.

2. Kannel WB, Wolf PA, Benjamin EJ, Levy D. Prevalence, incidence, prognosis, and predisposing conditions for atrial fibrillation: population-based estimates. The American journal of cardiology Oct 16 1998;82:2N-9N.

3. Schotten U, Verheule S, Kirchhof P, Goette A. Pathophysiological mechanisms of atrial fibrillation: a translational appraisal. Physiological reviews Jan 2011;91:265-325.

4. Nattel S. Newer developments in the management of atrial fibrillation. American heart journal Nov 1995;130:1094-1106.

5. Duffy HS, Wit AL. Is there a role for remodeled connexins in AF? No simple answers. Journal of molecular and cellular cardiology Jan 2008;44:4-13.

6. Spach MS, Heidlage JF, Barr RC, Dolber PC. Cell size and communication: role in structural and electrical development and remodeling of the heart. Heart rhythm : the official journal of the Heart Rhythm Society Oct 2004;1:500-515.

7. Spach MS, Boineau JP. Microfibrosis produces electrical load variations due to loss of side-to-side cell connections: a major mechanism of structural heart disease arrhythmias. Pacing and clinical electrophysiology : PACE Feb 1997;20:397-413.

8. Hocini M, Ho SY, Kawara T, Linnenbank AC, Potse M, Shah D, Jais $P$, Janse MJ, Haissaguerre M, De Bakker JM. Electrical conduction in canine pulmonary veins: electrophysiological and anatomic correlation. Circulation May 21 2002;105:2442-2448.

9. Eckstein J, Zeemering S, Linz D, Maesen B, Verheule S, van Hunnik A, Crijns H, Allessie MA, Schotten U. Transmural conduction is the predominant mechanism of breakthrough during atrial fibrillation: evidence from simultaneous endo-epicardial high-density activation mapping. Circulation Arrhythmia and electrophysiology Apr 2013;6:334-341.

10. Eckstein J, Maesen B, Linz D, Zeemering S, van Hunnik A, Verheule S, Allessie M, Schotten U. Time course and mechanisms of endo-epicardial electrical dissociation during atrial fibrillation in the goat. Cardiovascular research Mar 1 2011;89:816-824.

11. Maesen B, Zeemering S, Afonso C, et al. Rearrangement of atrial bundle architecture and consequent changes in anisotropy of 
conduction constitute the 3-dimensional substrate for atrial fibrillation. Circulation Arrhythmia and electrophysiology Oct 2013;6:967-975.

12. Allessie MA, de Groot NM, Houben RP, Schotten U, Boersma E, Smeets JL, Crijns HJ. Electropathological substrate of longstanding persistent atrial fibrillation in patients with structural heart disease: longitudinal dissociation. Circulation Arrhythmia and electrophysiology Dec 2010;3:606-615.

13. de Groot NM, Houben RP, Smeets JL, Boersma E, Schotten U, Schalij MJ, Crijns H, Allessie MA. Electropathological substrate of longstanding persistent atrial fibrillation in patients with structural heart disease: epicardial breakthrough. Circulation Oct 26 2010;122:1674-1682.

14. Verheule S, Eckstein J, Linz D, Maesen B, Bidar E, Gharaviri A, Schotten U. Role of endo-epicardial dissociation of electrical activity and transmural conduction in the development of persistent atrial fibrillation. Progress in biophysics and molecular biology Aug 2014;115:173-185.

15. Schuessler RB, Kawamoto T, Hand DE, Mitsuno M, Bromberg BI, Cox JL, Boineau JP. Simultaneous epicardial and endocardial activation sequence mapping in the isolated canine right atrium. Circulation Jul 1993;88:250-263.

16. Haissaguerre M, Lim KT, Jacquemet V, Rotter M, Dang L, Hocini M, Matsuo S, Knecht S, Jais P, Virag N. Atrial fibrillatory cycle length: computer simulation and potential clinical importance. Europace : European pacing, arrhythmias, and cardiac electrophysiology : journal of the working groups on cardiac pacing, arrhythmias, and cardiac cellular electrophysiology of the European Society of Cardiology Nov 2007;9 Suppl 6:vi64-70.

17. Ruchat P, Dang L, Virag N, Schlaepfer J, von Segesser LK, Kappenberger L. A biophysical model of atrial fibrillation to define the appropriate ablation pattern in modified maze. European journal of cardio-thoracic surgery : official journal of the European Association for Cardio-thoracic Surgery Jan 2007;31:65-69.

18. Uldry L, Virag N, Lindemans F, Vesin JM, Kappenberger L. Atrial septal pacing for the termination of atrial fibrillation: study in a biophysical model of human atria. Europace : European pacing, arrhythmias, and cardiac electrophysiology : journal of the working groups on cardiac pacing, arrhythmias, and cardiac 
cellular electrophysiology of the European Society of Cardiology Nov 2012;14 Suppl 5:v112-v120.

19. Zhao J, Butters TD, Zhang H, Pullan AJ, LeGrice IJ, Sands GB, Smaill BH. An image-based model of atrial muscular architecture: effects of structural anisotropy on electrical activation. Circulation Arrhythmia and electrophysiology Apr 2012;5:361370.

20. Aslanidi OV, Colman MA, Stott J, Dobrzynski H, Boyett MR, Holden AV, Zhang H. 3D virtual human atria: A computational platform for studying clinical atrial fibrillation. Progress in biophysics and molecular biology Oct 2011;107:156-168.

21. Krueger MW, Severi S, Rhode K, Genovesi S, Weber FM, Vincenti A, Fabbrini P, Seemann G, Razavi R, Dossel O. Alterations of atrial electrophysiology related to hemodialysis session: insights from a multiscale computer model. Journal of electrocardiology MarApr 2011;44:176-183.

22. Colman MA, Aslanidi OV, Kharche S, Boyett MR, Garratt C, Hancox JC, Zhang H. Pro-arrhythmogenic effects of atrial fibrillation-induced electrical remodelling: insights from the three-dimensional virtual human atria. The Journal of physiology Sep 1 2013;591:4249-4272.

23. Kuijpers NH, Potse M, van Dam PM, ten Eikelder HM, Verheule S, Prinzen FW, Schotten U. Mechanoelectrical coupling enhances initiation and affects perpetuation of atrial fibrillation during acute atrial dilation. Heart rhythm : the official journal of the Heart Rhythm Society Mar 2011;8:429-436.

24. Vigmond EJ, Ruckdeschel R, Trayanova N. Reentry in a morphologically realistic atrial model. Journal of cardiovascular electrophysiology Sep 2001;12:1046-1054. 


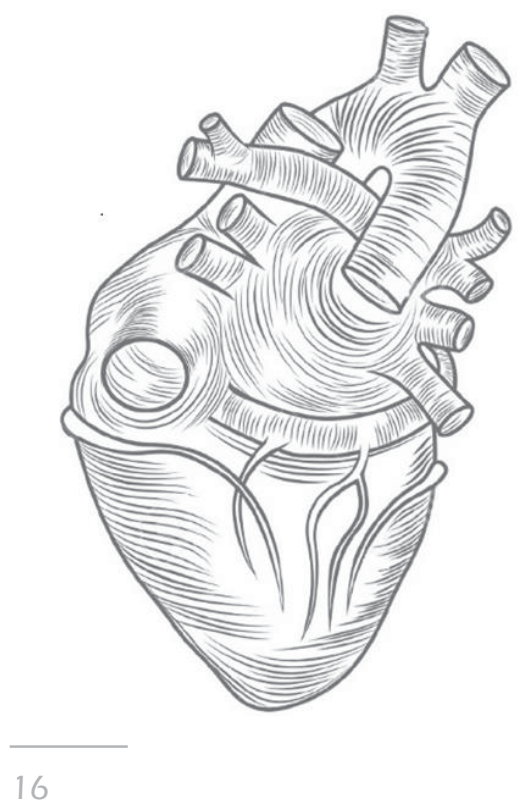




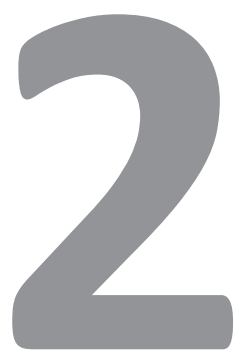

\section{A Computer Model of Endo-Epicardial Electrical Dissociation and Transmural Conduction during Atrial Fibrillation}

Ali Gharaviri

Sander Verheule

Jens Eckstein,

Mark Potse

Nico H.L. Kuijpers

Ulrich Schotten

Published in Europace. 2012 Nov;14

Suppl 5:v10-v16. doi:

0.1093/europace/eus270

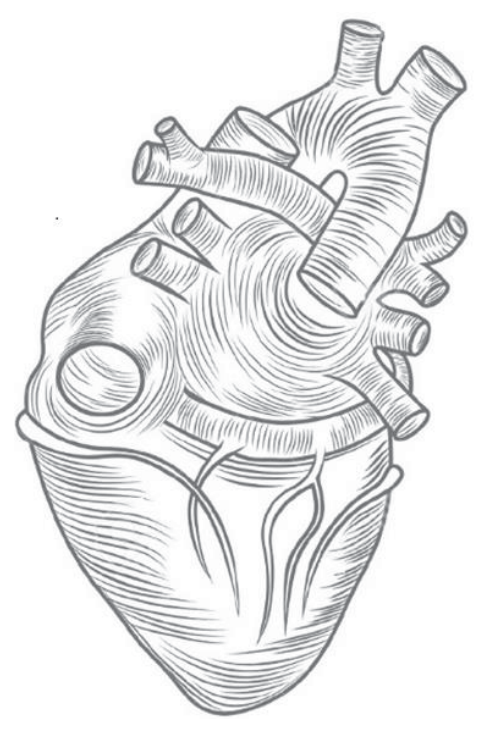




\section{Abstract}

\section{Background:}

Structural alterations during atrial fibrillation $(\mathrm{AF})$ not only lead to electrical dissociation within the epicardial layer, but also between the epicardial layer and the endocardial bundle network. The aim of the study was to investigate the role of transmural conduction in the stability of AF episodes using a dual-layer computer model.

\section{Methods:}

A 'proof-of-principle' dual-layer model was developed in which connections between the layers can be introduced or removed at any time during the simulation. Using an S1-S2 protocol, a spiral wave was initiated in one of the layers, which degenerated into a complex AF pattern after connection with the other layer at six randomly chosen sites. After six seconds, connections were either retained (dual-layer simulations) or removed (single layer simulations).

\section{Results:}

Dual-layer simulations were more complex, as indicated by the higher number of waves and phase singularities. Tracking waves through both layers revealed that the number of waves in duallayer simulations was significantly higher than in the single layer simulations, reflecting more opportunities for reentry and a concomitant increase in AF stability. In the dual-layer model, only $12 \%$ of the AF episodes died out within six seconds, while $59 \%$ died out in the single layer model.

\section{Conclusions:}

AF patterns are more complex and AF episodes are more stable in a dual-layer model. This study indicates an important role for endoepicardial conduction for stabilization of AF. 


\section{Introduction}

Atrial fibrillation (AF) is characterized by multiple fibrillation waves randomly propagating through the complex anatomy of the atrial wall. The development of the substrate for AF goes along with an increase in the incidence of conduction block and thus an increase in the number of fibrillation waves. Simultaneous mapping of the epicardial layer and the endocardial bundle network in goats revealed a surprisingly large degree of dissociation between these two layers. Goats with acutely induced AF showed 18\% dissociation of electrical activity, while dissociation had increase to $40 \%$ in goats with persistent AF and to $70 \%$ in goats with permanent AF. ${ }^{1}$ This suggests that fibrillatory conduction acquires a much more 3dimensional character with progressive structural remodeling than was previously anticipated. To address the question whether endoepicardial dissociation increases AF stability, we have developed a proof-of-principle computer model of two interconnected layers. $\mathrm{AF}$ in itself and structural heart diseases cause structural alterations in the atria favoring local conduction heterogeneity. The most important structural alterations underlying these conduction disturbances are atrial fibrosis, hypertrophy of atrial myocytes, amyloidosis, and possibly gap junction alterations. ${ }^{2}$ Recently, the electrophysiological correlate of this process of structural remodeling was demonstrated to consist of electrical dissociation between muscle bundles. ${ }^{3,4}$ Electrical dissociation leads to more and narrower wavefronts, greatly increasing the stability of AF. Structural alterations do not only lead to electrical dissociation within the epicardial layer, but also between the epicardial layer and the endocardial bundle network. ${ }^{1,5}$ Differences in electrical activity between epi- and endocardium allow fibrillation waves to conduct transmurally, causing breakthrough in the contralateral layer. This hypothesis is supported by the observation that the incidence of breakthrough has been shown to be much higher in structurally remodeled atria than in normal atria, both in patients ${ }^{6}$ and in animal models of AF. As endo-epicardial dissociation of electrical activity can be regarded as the 'conditio sine qua non' for transmural conduction, this observation implies that endo-epicardial 
dissociation would be more pronounced in structurally remodeled atria. Indeed, diffences in activation time and direction of conduction between the epicardium and endocardium did increase progressively during 6 months of AF in goats. ${ }^{1}$

Despite strong evidence for the 3-dimensional character of the conduction pattern during $\mathrm{AF}$, computer models of transmural conduction and endo-epicardial dyssynchrony are lacking. We therefore developed a computer model allowing 3-dimensional conduction during AF. In this model we have studied the effect of transmural conduction on the stability of the fibrillatory process and the dynamic properties of fibrillation waves.

\section{Methods}

\subsection{Model}

To investigate the role of transmural connections in AF stability and complexity, a novel computer model was developed. Atrial tissue was described by a monodomain reaction-diffusion model comprising two layers of $4 \times 4 \mathrm{~cm}$. Each of the layers was composed of $400 \times 400$ segments of size $0.01 \times 0.01 \mathrm{~cm}$ and atrial membrane behavior for each segment was based on the CourtemancheRamirez-Nattel ${ }^{7}$ model. The monodomain model is described by

$$
\chi\left(C_{m} \frac{\partial V_{m}}{\partial t}+I_{\text {ion }}+I_{\text {stim }}\right)=-\nabla \cdot\left(\mathbf{G} \nabla V_{m}\right)
$$

where $\chi$ is membrane surface-to-volume ratio, $C_{m}$ is membrane capacitance, $I_{i o n}$ is ionic membrane current, $I_{\text {stim }}$ is externally applied stimulus current, and $\mathbf{G}$ is the conductivity tensor. ${ }^{8}$ In the present study, $\chi=2000 \mathrm{~cm}-1, C_{m}=1 \mu \mathrm{F} / \mathrm{cm} 2$, and conductivities were the same in both directions, $\sigma_{x}=\sigma_{y}=0.5 \mathrm{mS} / \mathrm{cm}$, which implicates isotropic tissue. To incorporate changes in ionic membrane currents as observed in atrial fibrillation, maximum conductivity for transient outward potassium current ( $\mathrm{I}_{\text {to }}$ ) was reduced with $60 \%$, maximum conductivity for L-type calcium 
current $\left(\mathrm{I}_{\mathrm{Ca}, \mathrm{L}}\right)$ was reduced with $65 \%$, and maximum conductivity for inward rectifier potassium current $\left(\mathrm{I}_{\mathrm{K} 1}\right)$ was increased with $100 \%{ }^{9}$ Electrical connections between the two layers were incorporated by adding resistances between opposing segments in a circular area with radius $0.1 \mathrm{~cm}$ (conductivity $\sigma_{z}=0.5 \mathrm{mS} / \mathrm{cm}$ ). Connections can be introduced or removed at any time during the simulation.

\subsection{Simulation protocol}

To investigate the effect of transmural connections on fibrillatory behavior and stability of AF episodes the following simulation protocol was applied:

1. In one of the layers, a spiral wave was initiated using an S1-S2 protocol $^{10}$, while the other layer was not stimulated (Fig.1B).

2. The situation, one second after the start of the simulation, was used as the starting condition for an additional 6 seconds of simulation time in which the layers were connected by 6 connections. To exclude a bias caused by a particular geometry of the connection points, 8 separate simulations were performed with different sets of six randomly chosen connections. Connection points for each simulation were chosen such that two connections were at least $0.2 \mathrm{~cm}$ apart. Since clustering of connection sites may effectively reduce the number of connection sites, each configuration of the connection sites was visually inspected and rejected in case of clustering. All simulations were continued for 6 more seconds.

3. 7 seconds after initiation, all simulations continued for another 6 seconds, either (a) without changing the connections or (b) after removing all connections.

The simulations of step $3 a$ formed a group of 8 dual-layer simulations and the simulations of step $3 \mathrm{~b}$ together with the continued simulation of step 1 formed a group of 17 single-layer simulations. 


\subsection{Analysis}

AF complexity and stability was analyzed for all simulations. Stability was assessed by determining whether the tissue was still electrical active (stable AF) or quiescent (non-stable AF) after 6s of simulation time. We determined four measures of complexity each $1 \mathrm{~ms}$ of simulation time:

1. Number of waves;

2. Number of phase singularities;

3. Lifespan of phase singularities;

4. Lifespan of wave chains.

\subsubsection{Detecting Waves}

A wave was defined as a contiguous area in which all segments have membrane potentials above the excitation threshold of $60 \mathrm{mV}$. The number of waves was calculated for each $1 \mathrm{~ms}$ during the entire simulation time.

\subsubsection{Detecting and tracking Phase Singularities}

A phase singularity (PS), which forms the tip of a spiral wave, was defined using a transform of membrane potential distribution into phase. The algorithm used to detect PSs was based on the algorithm proposed by Zou et. al. ${ }^{11}$ Since the tip of the spiral wave is surrounded by tissue in all phases of the action potential, PSs can be found by assigning an appropriate value representing the phase of the action potential as follows. For each segment at location $\vec{r}=(x, y)$, phase $\theta$ is defined at simulation time $t$ by

$$
\theta(\vec{r}, t)=\arctan \left(\frac{V_{m}(\vec{r}, t+\tau)-V^{*}}{V_{m}(\vec{r}, t)-V^{*}}\right),
$$

where $V_{m}$ is the membrane potential at location $\vec{r}$ and time $t, V^{*}=-$ $45 \mathrm{mV}$ is the average of $V_{m}(\vec{r}, t)$ when the tissue is not quiescent, 
and $\tau$ is a time window of $10 \mathrm{~ms}$. The resulting $\theta(\vec{r}, t)$ has a value between $\frac{-\pi}{2}$ and $\frac{\pi}{2}$.

The phase at the exact center of a PS is undefined whereas surrounding sites exhibit a continuous progression of verging phase values. Candidate PSs are found at locations at which the line integral of the gradient of phase around a site equals $\pm \pi$, i.e.,

$$
\vec{r} \mid \oint \nabla \theta d l= \pm \pi
$$

The number of phase singularities was calculated each $1 \mathrm{~ms}$ during the whole simulation time.

PSs were tracked in time and space as follows. The distance was calculated between all PSs detected at simulation time $t+1 \mathrm{~ms}$ and all PSs detected at simulation time $t$. If the minimum distance was less than $1 \mathrm{~mm}$, this PS obtained the same ID as the PS with minimum distance. If for a PS the minimum distance was larger than $1 \mathrm{~mm}$, the PS was considered as 'newborn PS' and obtained a new ID. By choosing $1 \mathrm{~mm}$ as a threshold, it was assumed that a PS cannot travel faster than $1 \mathrm{~m} / \mathrm{s}$.

\subsubsection{Detecting Breakthroughs}

A breakthrough (BT) is a wave that appears in one layer and cannot be linked directly to the propagation of other waves in that layer (Fig. 1C). BTs were detected as follows. Areas containing connection points were monitored each $1 \mathrm{~ms}$. If a new wave appeared in one of these areas and could not be related to the propagation of other waves in that layer, and it had a size at most the size of the connection area, it was marked as a candidate breakthrough. If a candidate breakthrough increased in size within the next $2 \mathrm{~ms}$, it was marked as a breakthrough. The moment that the breakthrough appeared as well as the location were saved for further analysis. 

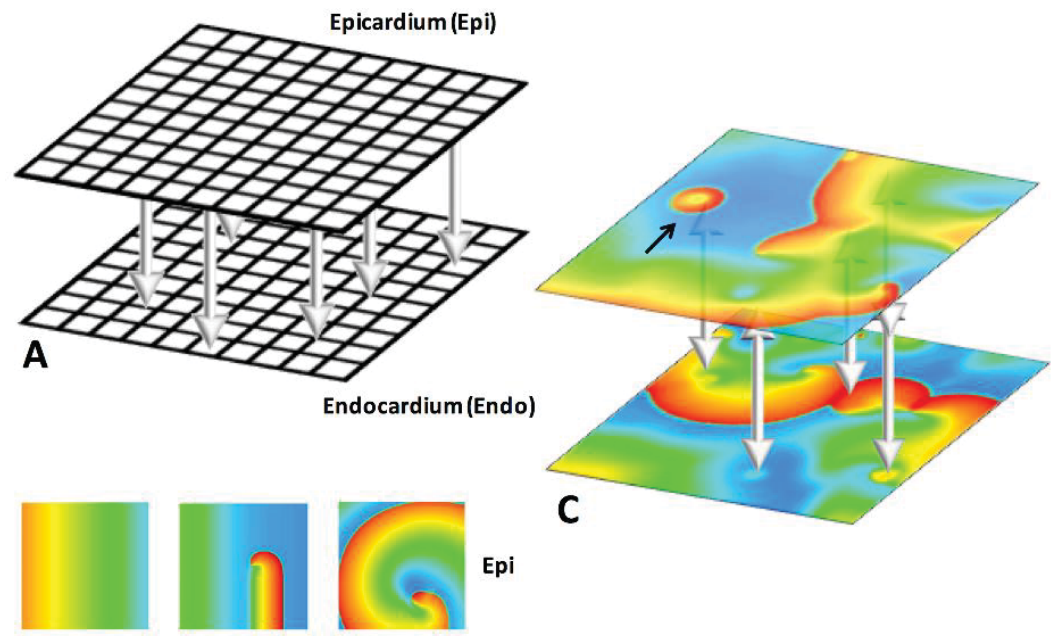

Epi
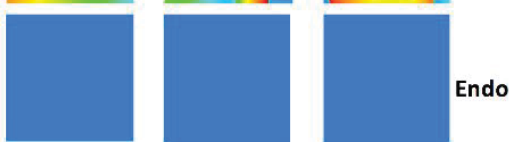

B

Figure 1. A) Model structure with two layers (epi- and endocardium) and transmural connections (gray arrows). B) Spiral wave initiation using S1-S2 protocol. C) Dissociation of electrical activity between the two layers and transmural conduction resulting in a breakthrough wave (black arrow).

\subsubsection{Wave Chain Tracking}

The propagation of fibrillation waves in our model was analyzed by tracking waves in time and space. If a wave passes a connection point in our model, the wave may propagate through that connection to the other layer if the tissue at the other side is excitable (Fig. 1C). Thus a breakthrough appears and the new wave is part of the same "wave chain". The temporal dynamics of waves can be described by three events:

- Generation: appearance of a new wave because a wave breaks up into two or more waves;

- Fusion: the fusion of two or more waves into one wave as they merge with each other;

- Extinction: the disappearance of a wave, because it hits a boundary or runs into unexcitable tissue. 
In this analysis, waves appearing in the other layer as a breakthrough were not considered 'newly generated' waves but rather as continuation of the same wave chain in the other layer. Waves were tracked in time by comparing AF patterns each $1 \mathrm{~ms}$, similar to the approach reported by Ten Tusscher et al. ${ }^{12}$ and Clayton et al. ${ }^{13}$ Waves were defined as contiguous areas in which the membrane potential exceeded $-60 \mathrm{mV}$. For all waves found at simulation time $t$, overlap (amount of segments) was computed with all waves found at simulation time $t+1$. If a wave at simulation time $t$ did not have overlap with any wave in simulation time $t+1$, the wave was considered to be extinguished. If a wave at simulation time $t+1$ had overlap with two or more waves at simulation time $t$, the waves were considered to be fused. If a wave at simulation time $t$ had overlap with two or more waves at simulation time $t+1$, a break-up occurred and a new wave was generated. Finally, if a wave appeared at simulation time $t+1$ and has no overlap with any wave at simulation time $t$, it means that a breakthrough occurred. In the latter case, overlap between the breakthrough wave and all waves from the other layer is computed to determine the source of the breakthrough.

Identification numbers (IDs) were assigned as follows. At simulation time $t=1$, all waves in both layers were detected and obtained an ID. If a wave was extinguished, the ID of that wave was not used again. If, because of break-up, one or more new waves were newly generated, the size of all daughter waves was calculated. The largest one retained the parent's ID, while the remaining waves obtained new IDs. If fusion occurred, the size of all waves that merged was calculated and the fused wave obtained the ID of the largest one. If a breakthrough occurred, the breakthrough wave obtained the ID of the wave in the contralateral layer that was determined to be the source of the breakthrough.

\subsection{Numerical methods and implementation}

The model used for the present simulation study was based on our previously published bi-domain model. ${ }^{14}$ The mono-domain equation was solved assuming no-flux boundary conditions using an explicit 
numerical scheme with time steps of $0.01 \mathrm{~ms}$ as previously described. ${ }^{15}$ Gating variables and intracellular ion concentrations were updated with time steps of $0.01 \mathrm{~ms}$ during the action potential upstroke and otherwise with time steps of $0.1 \mathrm{~ms} .{ }^{16}$ Gating variables were integrated using the Rush-Larsen method. ${ }^{17,18}$ The model was implemented in $\mathrm{C}++$ and executed on a normal workstation with Intel i7 processor and 6GB memory. It took 72 hours to simulate an AF episode of $6 \mathrm{~s}$ on the dual-layer model. Up to six simulations could run simultaneously on the multi-core processor without increase in computation time.

\subsection{Statistical analysis}

Statistical tests were performed to compare the two groups of simulations. (single-layer $\mathrm{n}=17$ and dual-layer $\mathrm{n}=8$ ). For each group, the average number of waves and the average number of PSs during the whole simulation time was calculated. In addition, the average of all PS lifespans and the average of all wave chain lifespans were calculated. Each data set was tested for normal distribution using the Kolmogorov-Smirnov test. An unpaired Student t-test was performed to compare normally distributed data sets and a non-parametric test (Mann-Whitney) was performed otherwise.

\section{Results}

\subsection{Stability}

The total number of simulations in the single-layer group was 17 and 8 in the dual-layer group. Membrane potentials of one of the simulations described in steps $2,3 a$, and $3 b$ of the simulation protocol are presented in Fig $2 \mathrm{~A}-\mathrm{C}$. In these simulations, $\mathrm{AF}$ persisted when the connections were retained (Fig $2 \mathrm{~A}$ and $2 \mathrm{C}$ ), while AF terminated in both layers when the connections were removed (Fig 2B). Fig. 2D illustrates a Kaplan-Meier curve showing the persistence of AF episodes in both groups. AF terminated in 
only one out of 8 dual-layer simulations, while it terminated in 10 out of 17 single-layer simulations.
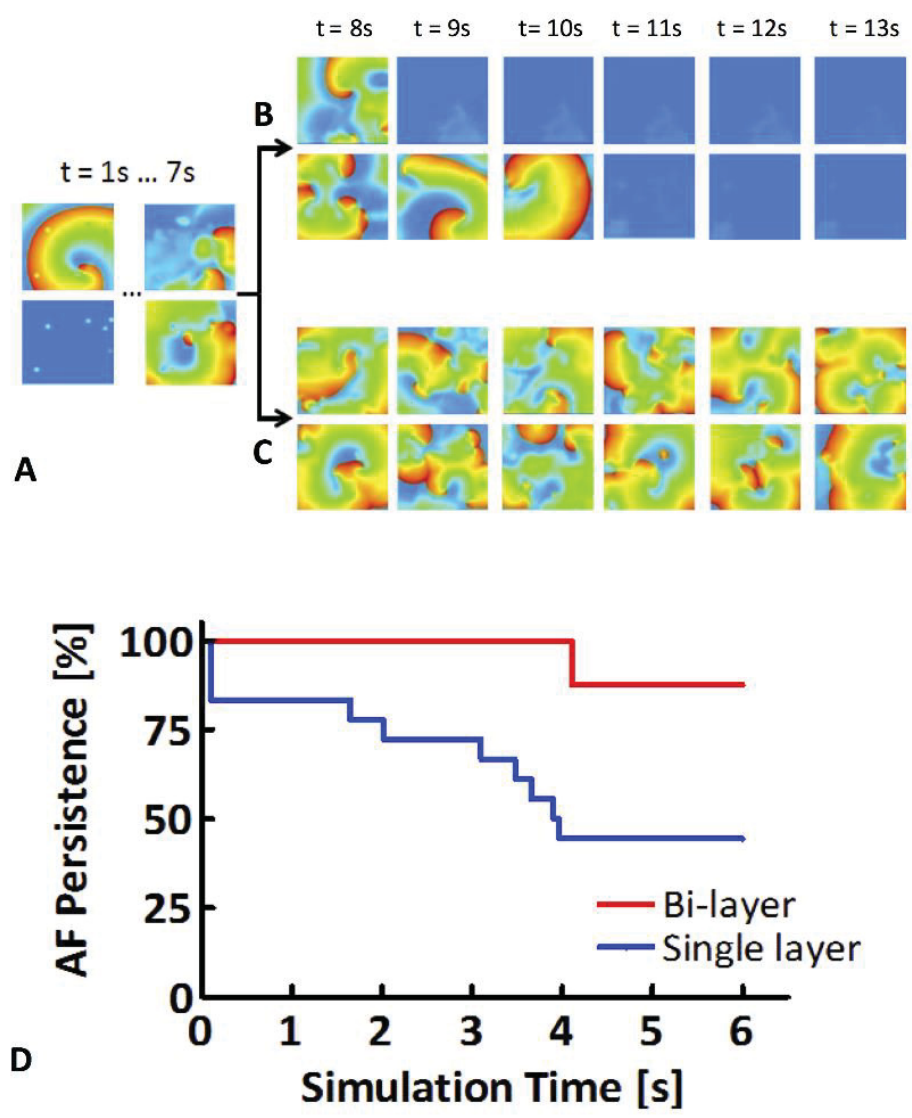

Figure 2. A) Membrane potential of a simulation at $t=1 \mathrm{~s}$ (introduction of connections) and $t=7 \mathrm{~s}$. B) Continuation of the simulation in A without changing connections (step $3 a$ in methods section 2.2). C) Continuation of the simulation in A after removing all connections (step 3b). D) Kaplan-Meier curve showing atrial fibrillation persistence in single and dual-layer simulations $(n=$ 17 for single layer and $n=8$ for double layer).

\subsection{Number of waves and phase singularities}

In all simulations, the number of waves and PSs was determined in both layers each $1 \mathrm{~ms}$. An overview of the analysis results is 
presented in Figure 3. The average number of simultaneously existing waves and PSs was significantly larger in the dual-layer model compared to the single-layer model (Fig. 3C).

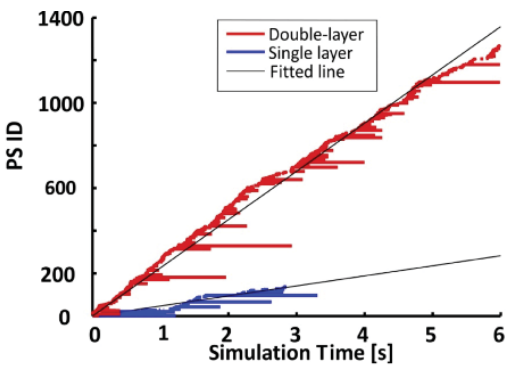

A

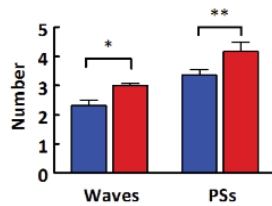

C

D

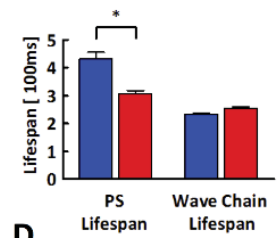

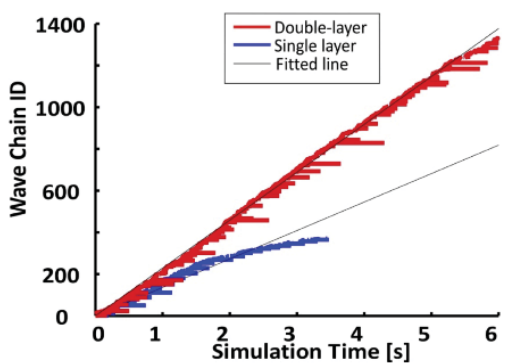

B

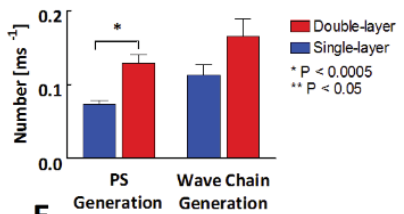

E

Figure 3. A) Phase singularity (PS) lifespans in dual-layer (red lines) and single-layer (blue lines) simulations and linear fit (black line), analyzed in the simulations shown figure 2B and 2C. B) Wave chain lifespans in dual-layer (red lines) and single-layer (blue lines) and linear fit (black line) from the simulations shown in figure $2 \mathrm{~B}$ and $2 \mathrm{C}$. C) Average number of waves and PSs during the time that $A F$ persisted for all simulations in each group D) Average of PSs and wave chain lifespans. E) Average of PS and wave generation rates.

\subsection{Phase singularities and wave chain tracking}

Figures $3 \mathrm{~A}$ and $3 \mathrm{~B}$ show $\mathrm{PSs}$ and wave chain life spans of single and dual-layer simulations. Over time, new PSs and waves are continuously formed, while old PSs and waves disappeared. Most of the PSs and waves were present for only a short period of time (many short lines), while a few PSs and waves lasted for a longer period (few long lines). The slopes of the line fitted through the starting points of lines representing PS and wave chain lifespan represent PS and wave generation rate. The average slope of the fitted lines for PSs and wave chain lifespans (black lines in Fig. 3A 
and $3 \mathrm{~B}$ ) is larger in the dual-layer model compared to the singlelayer model, indicating that the generation rate of wave chains and PSs in the dual-layer was higher (Fig. 3E). PSs and wave chains were tracked in time for both layers. Figure 3D shows that the average life span of PSs in the dual-layer simulation was shorter than in the single-layer while there was no difference in average lifespans of the wave chains.

\subsection{AF cycle length}

$\mathrm{AF}$ cycle length (AFCL) is the time interval between two consecutive activations, which are defined as the moment during the AP upstroke when the membrane potential crosses $-60 \mathrm{mV}$. In all simulations AFCL was calculated and averaged over time and space. Average AFCL in single-layer and dual-layer simulations were $148.5 \pm 4.6 \mathrm{~ms}$ and $143.5 \pm 3.6 \mathrm{~ms}$, respectively, and were not significantly different.

\section{Discussion}

A novel dual-layer computer model was developed to study the effect of endo-epicardial dissociation of electrical activity and transmural conduction on stability and complexity of AF. To the best of our knowledge, this is the first model simulating dyssynchronous electrical activity in two separate layers representing epicardium and endocardium - coupled by electrical connection points enabling transmural conduction and 'breakthrough' of fibrillation waves from one layer to the other. The study demonstrates that adding the third dimension to the substrate of AF greatly increases the stability of the simulated arrhythmia, which was explained by an increase in the number of coexisting waves due to a higher rate of wave generation. 


\subsection{Radial spread of activation as new type of simulated fibrillation wave}

Most computer models of AF treat the atrial wall as a twodimensional layer. ${ }^{19}$ Most 'three-dimensional' atrial models are three dimensional in the sense that a two-dimensional atrial sheet is folded into the shape of the atria, with two main cavities, atrial appendages and connections between the two atria by a septal ring. ${ }^{20-22}$ As a first step towards more realistic computer models of $A F$, a few computer models incorporated heterogeneities in atrial thickness. ${ }^{16,23-26}$ These studies have shown how spatial differences of anisotropy in specific muscle bundles affect normal conduction in the atria and that the heterogeneity of the atrial wall thickness significantly contributes to the stability of AF. However, these models did not take disruption of transmural connections and endoepicardial electrical dissociation (EED) into account. The novelty of our model is that it describes truly "three-dimensional' or 'transmural conduction' between an endocardial and an epicardial layer. Connection points were randomly distributed and chosen such that two connections were at least $0.2 \mathrm{~cm}$ apart. Each configuration of connection points was visually inspected and rejected in case of clustering. The important conceptual consequence is that this model introduces a new type of simulated fibrillation waves. After transmural conduction from one layer to the other, conduction showed radial spread of activation away from the electrical connection point, thereby resembling breakthroughs occurring during experimental and clinical AF.

So far phase singularities represent the exclusive type of reentrant fibrillation wave in most if not all computer models of AF. The correlate of phase singularities in experimental AF is spiral wave reentry with a rotor-shaped fibrillation wavefront conducting around an excitable yet unexcited core. Spiral wave reentry has been documented in animal models of AF with homogenous substrates and short refractory periods. ${ }^{27,} 28$ Heterogeneities in electrophysiological properties like fibrosis cause wave break and largely reduce the life span of spiral waves. In dogs with heart failure, atrial fibrosis results in the existence of multiple unstable 
rotors - a conduction pattern very similar to multiple wavelets. ${ }^{29}$ Importantly, spiral wave reentry in patients with $A F$ is a rare phenomenon. ${ }^{3}$ By contrast, numerous authors have documented breakthrough with radial spread of activation of fibrillation waves in experimental ${ }^{4,24,30}$ and clinical investigations. ${ }^{6,31,32}$ As breakthroughs contribute significantly to the perpetuation of $A F$, realistic computer models should be able to represent this type of conduction pattern. In the computer model described here, more than $40 \%$ of all waves are breakthroughs and the existence of these breakthroughs greatly increases the stability of the simulated fibrillatory process. Introduction of the third dimension can therefore be regarded as an important step towards more realistic computer models of AF.

\subsection{Effect of three-dimensional conduction on AF complexity and stability}

We demonstrate that AF was more stable in dual-layer simulations than in single-layer simulations. After $6 \mathrm{~s}$ simulation time, AF terminated in only 1 out of 8 dual-layer simulations (12.5\%), but in 10 out of $17(59 \%)$ single-layer simulations. The explanation for the increased stability of the AF episodes lies in an altered dynamic behavior of the AF waves. Both wave and PS numbers and lifespans were analyzed by monitoring breakthrough, splitting, fusion, and extinction of individual waves. The number of both waves and PS were significantly larger in the dual-layer model, which might well explain the enhanced AF stability. Most waves and PSs were short lived (shorter than $450 \mathrm{~ms}$ and $1000 \mathrm{~ms}$ in $90 \%$ of the wave chains and PSs, respectively), while less than $5 \%$ of PSs lived longer than $1300 \mathrm{~ms}$. Surprisingly, average PS lifespan during AF episodes in dual-layer simulations was significantly shorter compared to singlelayer simulations. The average lifespan of wave chains in the duallayer simulations was not altered. A possible explanation for the smaller survival rate of PSs in dual-layer simulations might be that breakthroughs reduce the space for PSs to meander and thereby disrupt the stable reentrant pattern of PS as has been described in an optical mapping study on fibrillating sheep atria. ${ }^{24}$ On the other 
hand, many breakthroughs appear in an area with heterogeneous distribution of excitability and as a result evolve into PSs. Indeed, we found that the rate of generation of new PSs was twice as large in the dual-layer as in the single-layer model. This increase in the wave generation rate is the main mechanism of the increase in the number of waves in the double layer model.

\subsection{Implications and possible applications of the model}

Endo-epicardial electrical dissociation and BT during AF have been described already in the 1990 s. ${ }^{30,31,33}$ However, only very recently it was recognized that endo-epicardial dissociation increases with the degree of remodeling ${ }^{1,5}$ and enhanced EED in remodeled atria offers an explanation of enhanced breakthrough rates in complex substrates for AF. As breakthroughs significantly contribute to the stability of AF, mathematical models investigating the perpetuation of AF or therapeutic interventions in AF need to implement this phenomenon. For example, a recent experimental study has demonstrated that flecainide greatly reduces the rate of breakthrough, which was attributable to a strong decrease in endoepicardial dissociation. ${ }^{25}$ Our model offers the opportunity to verify whether the antiarrhythmic action of flecainide can be explained by its effect on endo-epicardial dissociation and transmural conduction or whether other mechanisms related to changes in ectopic activity mediated by enhanced phosphorylation of the ryanodine receptor might play a role. ${ }^{34}$ Likewise, our model might be used to study mechanisms of AF perpetuation. By introducing changes in coupling between the two layers but also within the epicardial and endocardial layer, the effect of electrical dissociation on the 3-dimensional fibrillation pattern during AF can be studied. Clinical observations indicate that disruption of transmural connections leads to more breakthrough events, 6 although the number of possible pathways for breakthrough waves is decreased. Our model provides the ideal research environment to increase mechanistic insights in this phenomenon. With our model, changes in breakthrough rate during different stages of structural 
remodeling can be investigated and compared to clinical observations.

\section{Study limitations}

Our dual-layer model clearly is a simplified representation that does not reflect the anatomical complexities of the atria, the role of specialized structures like the pulmonary veins or heterogeneity in ionic membrane currents. It should be regarded as a proof-ofprinciple study, demonstrating that adding endo-epicardial dissociation of electrical activity and 'truly' three-dimensional conduction strongly increases complexity and stability of AF. Future studies will have to address the contribution of a more realistic anatomy and pathological changes in the atria to the perpetuation of $\mathrm{AF}$. 


\section{References:}

1. Eckstein J, Maesen B, Linz D, Zeemering S, van Hunnik A, Verheule S, Allessie M, Schotten U. Time course and mechanisms of endo-epicardial electrical dissociation during atrial fibrillation in the goat. Cardiovasc Res Mar 1 2011;89:816-824.

2. Schotten U, Verheule S, Kirchhof P, Goette A. Pathophysiological mechanisms of atrial fibrillation: a translational appraisal. Physiol Rev Jan 2011;91:265-325.

3. Allessie MA, de Groot NM, Houben RP, Schotten U, Boersma E, Smeets JL, Crijns HJ. Electropathological substrate of longstanding persistent atrial fibrillation in patients with structural heart disease: longitudinal dissociation. Circ Arrhythm Electrophysiol Dec 2010;3:606-615.

4. Verheule S, Tuyls E, van Hunnik A, Kuiper M, Schotten U, Allessie M. Fibrillatory conduction in the atrial free walls of goats in persistent and permanent atrial fibrillation. Circ Arrhythm Electrophysiol Dec 2010;3:590-599.

5. Everett THt, Wilson EE, Hulley GS, Olgin JE. Transmural characteristics of atrial fibrillation in canine models of structural and electrical atrial remodeling assessed by simultaneous epicardial and endocardial mapping. Heart Rhythm Apr 2010;7:506-517.

6. de Groot NM, Houben RP, Smeets JL, Boersma E, Schotten U, Schalij MJ, Crijns H, Allessie MA. Electropathological substrate of longstanding persistent atrial fibrillation in patients with structural heart disease: epicardial breakthrough. Circulation Oct 26 2010;122:1674-1682.

7. Courtemanche M, Ramirez RJ, Nattel S. Ionic mechanisms underlying human atrial action potential properties: insights from a mathematical model. Am J Physiol Jul 1998;275:H301-321.

8. Clayton $\mathrm{RH}$, Bernus $\mathrm{O}$, Cherry EM, Dierckx $\mathrm{H}$, Fenton $\mathrm{FH}$, Mirabella L, Panfilov AV, Sachse FB, Seemann G, Zhang H. Models of cardiac tissue electrophysiology: progress, challenges and open questions. Prog Biophys Mol Biol Jan 2011;104:22-48.

9. Bosch RF, Nattel S. Cellular electrophysiology of atrial fibrillation. Cardiovasc Res May 2002;54:259-269.

10. Leon LJ, Roberge FA, Vinet A. Simulation of two-dimensional anisotropic cardiac reentry: effects of the wavelength on the 
reentry characteristics. Ann Biomed Eng Nov-Dec 1994;22:592609.

11. Zou R, Kneller J, Leon LJ, Nattel S. Development of a computer algorithm for the detection of phase singularities and initial application to analyze simulations of atrial fibrillation. Chaos Sep 2002;12:764-778.

12. Ten Tusscher KH, Hren R, Panfilov AV. Organization of ventricular fibrillation in the human heart. Circ Res Jun 22 2007;100:e87-101.

13. Clayton RH, Holden AV. A method to quantify the dynamics and complexity of re-entry in computational models of ventricular fibrillation. Phys Med Biol Jan 21 2002;47:225-238.

14. Kuijpers $\mathrm{NH}$, Keldermann RH, Arts T, Hilbers PA. Computer simulations of successful defibrillation in decoupled and nonuniform cardiac tissue. Europace Sep 2005;7 Suppl 2:166-177.

15. Kuijpers NH, Keldermann RH, ten Eikelder HM, Arts T, Hilbers PA. The role of the hyperpolarization-activated inward current If in arrhythmogenesis: a computer model study. IEEE Trans Biomed Eng Aug 2006;53:1499-1511.

16. Kuijpers NH, Potse M, van Dam PM, ten Eikelder HM, Verheule S, Prinzen FW, Schotten U. Mechanoelectrical coupling enhances initiation and affects perpetuation of atrial fibrillation during acute atrial dilation. Heart Rhythm Mar 2011;8:429-436.

17. Rush S, Larsen H. A practical algorithm for solving dynamic membrane equations. IEEE Trans Biomed Eng Jul 1978;25:389392.

18. Courtemanche M, Ramirez RJ, Nattel S. Ionic mechanisms underlying human atrial action potential properties: insights from a mathematical model. Am J Physiol Jul 1998;275:H301-321.

19. Kneller J, Zou R, Vigmond EJ, Wang Z, Leon LJ, Nattel S. Cholinergic atrial fibrillation in a computer model of a twodimensional sheet of canine atrial cells with realistic ionic properties. Circ Res May 17 2002;90:E73-87.

20. Jacquemet $\mathrm{V}$, Virag $\mathrm{N}$, Kappenberger $\mathrm{L}$. Wavelength and vulnerability to atrial fibrillation: Insights from a computer model of human atria. Europace Sep 2005;7 Suppl 2:83-92.

21. Jacquemet V, Virag N, Ihara Z, Dang L, Blanc O, Zozor S, Vesin JM, Kappenberger L, Henriquez C. Study of unipolar electrogram morphology in a computer model of atrial fibrillation. J Cardiovasc Electrophysiol Oct 2003;14:S172-179. 
22. Jacquemet V, Kappenberger L, Henriquez CS. Modeling atrial arrhythmias: impact on clinical diagnosis and therapies. IEEE Rev Biomed Eng 2008;1:94-114.

23. David M. Harrild CSH. A Computer Model of Normal Conduction in the Human Atria. Circ Res September 29, 2000 2000;87:e25e36.

24. Yamazaki M, Mironov S, Taravant C, Brec J, Vaquero LM, Bandaru K, Avula UM, Honjo H, Kodama I, Berenfeld O, Kalifa J. Heterogeneous atrial wall thickness and stretch promote scroll waves anchoring during atrial fibrillation. Cardiovasc Res Feb 5 2012.

25. Eckstein J. The Three-Dimentional Substrate of Atrial Fibrillation in the Goat: A Simultaneous Endo-epicardial Mapping Study. Maastricht: Physiology Department, Maastricht University; 2012.

26. Seemann G, Hoper C, Sachse FB, Dossel O, Holden AV, Zhang H. Heterogeneous three-dimensional anatomical and electrophysiological model of human atria. Philos Transact A Math Phys Eng Sci Jun 15 2006;364:1465-1481.

27. Allessie MA, Bonke FI, Schopman FJ. Circus movement in rabbit atrial muscle as a mechanism of trachycardia. Circ Res Jul 1973;33:54-62.

28. Mandapati R, Skanes A, Chen J, Berenfeld O, Jalife J. Stable microreentrant sources as a mechanism of atrial fibrillation in the isolated sheep heart. Circulation Jan 18 2000;101:194-199.

29. Spach MS. Mounting evidence that fibrosis generates a major mechanism for atrial fibrillation. Circ Res Oct 12 2007;101:743745.

30. Gray RA, Pertsov AM, Jalife J. Incomplete reentry and epicardial breakthrough patterns during atrial fibrillation in the sheep heart. Circulation Nov 15 1996;94:2649-2661.

31. Holm M, Johansson R, Brandt J, Luhrs C, Olsson SB. Epicardial right atrial free wall mapping in chronic atrial fibrillation. Documentation of repetitive activation with a focal spread--a hitherto unrecognised phenomenon in man. Eur Heart $\mathrm{J}$ Feb 1997;18:290-310.

32. Konings KTS, Kirchhof CJHJ, Smeets JRLM, Wellens HJJ, Penn OC, Allessie MA. High-Density Mapping of Electrically-Induced Atrial-Fibrillation in Humans. Circulation Apr 1994;89:1665-1680.

33. Schuessler RB, Kawamoto T, Hand DE, Mitsuno M, Bromberg BI, Cox JL, Boineau JP. Simultaneous epicardial and endocardial 
activation sequence mapping in the isolated canine right atrium. Circulation Jul 1993;88:250-263.

34. Greiser M, Neuberger HR, Harks E, et al. Distinct contractile and molecular differences between two goat models of atrial dysfunction: AV block-induced atrial dilatation and atrial fibrillation. J Mol Cell Cardiol Mar 2009;46:385-394. 


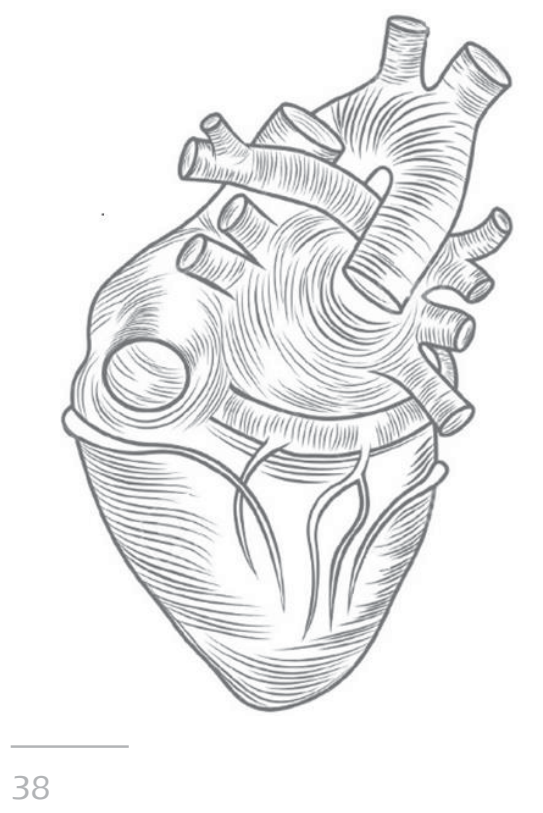




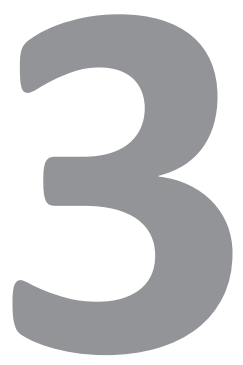

How Disruption of Endo-epicardial Electrical Connections Enhances Endo-epicardial Conduction during Atrial Fibrillation

\section{Ali Gharaviri}

Sander Verheule

Jens Eckstein,

Mark Potse

Pawel Kuklik

Nico H.L. Kuijpers

Ulrich Schotten

Dept. of Physiology, Maastricht University, Maastricht, The Netherlands.

Published in Europcace.2016 Feb; doi:10.1093/europace/eu445

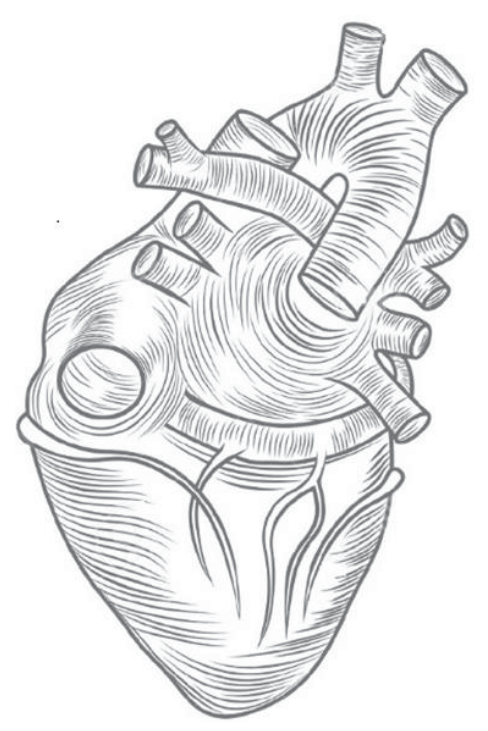




\section{Abstract}

\section{Background:}

Loss of side-to-side electrical connections between atrial muscle bundles is thought to underlie conduction disturbances predisposing to atrial fibrillation (AF). Putatively, disruption of electrical connections occurs not only within the epicardial layer but also between the epicardial layer and the endocardial bundle network, thus impeding transmural conductions ("breakthroughs"). However, both clinical and experimental studies have shown an enhancement of breakthroughs during later stages of AF. We tested the hypothesis that endo-epicardial uncoupling enhances endoepicardial electrical dyssynchrony, breakthrough rate (BTR), and AF stability.

\section{Methods:}

In a novel dual-layer computer model of the human atria, $100 \%$ connectivity between the two layers served as healthy control. Atrial structural remodeling was simulated by reducing the number of connections between the layers from 96 to 6 randomly chosen locations.

\section{Results:}

With progressive elimination of connections, AF stability increased. Reduction of the number of connections from 96 to 24 resulted in an increase in endo-epicardial dyssynchrony from $6.6 \pm 1.9 \%$ to $24.6 \pm 1.3 \%$, with a concomitant increase in BTR. A further reduction to 12 and 6 resulted in more pronounced endo-epicardial dyssynchrony of $34.4 \pm 1.15 \%$ and $40.2 \pm 0.52 \%$ but with BTR reduction. This biphasic relationship between endo-epicardial coupling and BTR was found independently from whether AF was maintained by reentry or by ectopic focal discharges. 


\section{Conclusions:}

Loss of endo-epicardial coupling increases AF stability. There is a biphasic relation between endo-epicardial coupling and BTR. While at high degrees of endo-epicardial connectivity the BTR is limited by the endo-epicardial synchronicity, at low degrees of connectivity it is limited by number of endo-epicardial connections. 


\section{Introduction}

Several mechanisms have been suggested to explain the increasing stability of atrial fibrillation (AF) over time. Disruption of electrical coupling between muscle bundles, resulting in narrower and thus more fibrillation waves, is considered as one of the main mechanisms contributing to AF stability in structurally remodeled atria. $^{1-4}$ The anatomy of the atrial wall has been demonstrated to significantly determine conduction patterns of fibrillation waves during AF. ${ }^{5}$ For example, Gray et al. showed that the direction of atrial muscle bundles correlates well with the location of block lines and breakthrough in right atrium. ${ }^{6}$ Similarly, Wu et al. demonstrated an effect of orientation of pectinate muscles on fibrillation patterns and observed transmural conduction in the area of pectinate muscles. ${ }^{7}$ De Groot et al. found increases both in the number of fibrillation waves and in incidence of epicardial breakthroughs in patients with longstanding AF compared to patients with acute AF, suggesting that the development of the substrate for AF goes along with increasing incidence of conduction from the sub-epicardial layer to the endocardial bundle network and vice versa. This concept of three-dimensional conduction of fibrillation waves during AF was recently confirmed by several animal studies using simultaneous mapping of the epicardial layer and the endocardial bundle network. ${ }^{6-9-11}$ While these studies conclusively demonstrate transmural conduction in the atrial wall, they leave open several important conceptual questions.

In particular they could not answer the question what drives threedimensional conduction during AF. In structurally remodeled atria, disruption of electrical connections not only occurs between the muscle bundles within the epicardial layer but also between the epicardial layer and the endocardial bundle network. This should enhance endo-epicardial conduction block and thus impede endoepicardial conduction. However, the epicardial breakthrough rate is higher in goats and patients with persistent AF compared to acute AF. ${ }^{2,11,12}$ In a previous simulation study, ${ }^{13}$ we used a new dual-layer computer model of AF to address the question whether dissociation of endo-epicardial electrical activity results in increased AF 
stability. In the present study, we test the hypothesis that progressive loss of endo-epicardial electrical connections increases the degree of endo-epicardial dissociation of electrical activity and that enhanced degree of endo-epicardial dissociation results in an enhanced breakthrough rate and a more complex fibrillation pattern.

\section{Methods}

\subsection{Model}

\subsubsection{Model structure}

A recent study by Eckstein et al. revealed an increase in electrical activity dissociation between epicardial layer and endocardial bundle network due to progressive uncoupling between these two layers..$^{10}$ In order to model this phenomenon we chose a novel duallayer computer model with varying degrees of electrical coupling between two layers (each layer is representation of either the endocardial or the epicardial layer). Each layer was modeled with a mono-domain reaction-diffusion model of a size of $4 \mathrm{~cm} \times 4 \mathrm{~cm}$ and composed of $400 \times 400$ segments with a size of $0.01 \mathrm{~cm} \times 0.01 \mathrm{~cm}$. Since specific connectivity between remodeled epicardial and endocardial layer is not known, we chose a randomly distributed set of circular connection points between both layers. However, as connection point distribution may have a large impact on conduction patterns we performed simulations with more heterogeneous connection distributions (see supplemental material).

Electrical coupling between the two layers was implemented by adding an ohmic conductor between opposing segments at so called connection points. The radii of the connection points were chosen so that proper transmural conduction was obtained (Figure $1 \mathrm{~A}) .^{13}$ 


\subsubsection{Atrial Cell Model}

Ionic currents and calcium handling for each segment was described by the Courtemanche-Ramirez-Nattel model. ${ }^{14}$ Conductivities were the same in both directions, $\sigma_{\mathrm{x}}=\sigma_{\mathrm{y}}=0.5 \mathrm{mS} / \mathrm{cm}$, which implies isotropic tissue. Total ionic membrane current was given by;

$\mathrm{I}_{\mathrm{ion}}=\mathrm{I}_{\mathrm{Na}}+\mathrm{I}_{\mathrm{K} 1}+\mathrm{I}_{\mathrm{to}}+\mathrm{I}_{\mathrm{Kur}}+\mathrm{I}_{\mathrm{Kr}}+\mathrm{I}_{\mathrm{Ks}}+\mathrm{I}_{\mathrm{Ca}, \mathrm{L}}+\mathrm{I}_{\mathrm{p}, \mathrm{Ca}}+\mathrm{I}_{\mathrm{NaK}}+\mathrm{I}_{\mathrm{NaCa}}+\mathrm{I}_{\mathrm{b}, \mathrm{Na}}+$ $\mathrm{I}_{\mathrm{b}, \mathrm{Ca}}$

where $\mathrm{I}_{\mathrm{Na}}$ is fast inward $\mathrm{Na}^{+}$current, $\mathrm{I}_{\mathrm{K} 1}$ is inward rectifier $\mathrm{K}^{+}$current, $\mathrm{I}_{\text {to }}$ transient outward $\mathrm{K}^{+}$current, $\mathrm{I}_{\mathrm{kur}}$ is ultrarapid delayed rectifier $\mathrm{K}^{+}$ current, $I_{k r}$ is rapid delayed rectifier current, $I_{\mathrm{Ks}_{\mathrm{s}}}$ is slow delayed rectifier $\mathrm{K}^{+}$current, $\mathrm{I}_{\mathrm{Ca}, \mathrm{L}}$ is $\mathrm{L}$-type $\mathrm{Ca}^{2+}$ current, $\mathrm{I}_{\mathrm{p}, \mathrm{Ca}}$ is $\mathrm{Ca}^{2+}$ pump current, $\mathrm{I}_{\mathrm{NaK}}$ is $\mathrm{Na}^{+}-\mathrm{K}^{+}$pump current, $\mathrm{I}_{\mathrm{NaCa}}$ is $\mathrm{Na}^{+} / \mathrm{Ca}^{2+}$ exchanger current, and $\mathrm{I}_{b, \mathrm{Na}}$ and $\mathrm{I}_{\mathrm{b}, \mathrm{Ca}}$ are background $\mathrm{Na}^{+}$and $\mathrm{Ca}^{2+}$ currents. To incorporate changes in ionic currents as observed in atrial fibrillation, conductivities for $I_{t o}, I_{C a, L}$, and $I_{K 1}$ were set at $40 \%, 35 \%$, and $200 \%$ of the default values, respectively. ${ }^{3}$

\subsection{Simulation protocols}

To demonstrate that the quantitative relation between the breakthrough rate, the degree of endo-epicardial dyssynchrony, and the number of endo-epicardial connections is independent from the mechanisms driving $A F$, the arrhythmia was maintained by $A$ ) spiral waves and $B$ ) random focal activation mimicking continuous ectopic focal discharges.

A) AF driven by spiral waves

To investigate the effect of the number of connection points on fibrillatory behavior, the following simulation protocol was applied (Figure 1C):

1. In one layer, a spiral wave was initiated using an S1-S2 protocol, ${ }^{15-17}$ while the other layer was quiescent, as 
described in our previous study. ${ }^{13}$ The simulation was continued for 1 second.

2. One second after the start of the simulation, 6 connection points were added at randomly chosen sites. These sites were chosen such that two connection points were at least $0.15 \mathrm{~cm}$ apart. To exclude possible bias resulting from a particular geometry of connection points, 8 different sets of 6 randomly chosen sites were created. For each of the 8 configurations, the simulation of step 1 was continued for 6 more seconds. This resulted in 8 different simulations that were used to initialize the simulations in the next step.

3. The 8 simulations from step 2 were continued for another 6 seconds, either without changing the connection points or after adding randomly chosen connection points (at least $0.15 \mathrm{~cm}$ apart). Thus, the total number of connections was $6,12,24,48$ or 96 . In addition, each simulation from step 2 was continued with $100 \%$ connectivity, i.e., all opposing segments in the two layers were connected to each other. As described above, steps 2 and 3 were performed 8 times such that in total 48 simulation runs were performed in step 3 .

B) AF driven by random ectopy

1. Both layers were paced at randomly chosen pacing cites distributed over both layers. The model was paced at random time steps. The frequency of ectopy was chosen in a way that an AF cycle length around $200 \mathrm{~ms}$ was achieved.

2. Step 1 was done for all different number of connections (6, $12,24,48,96$, and $100 \%$ connectivity) for eight randomly distributed connections and simulations continued for 6 seconds.

In our model, 6 and 12 connections represented severely remodeled atria; 24, 48, and 96 connections represented moderately remodeled atria; and $100 \%$ connectivity represented a healthy atrium. 
A

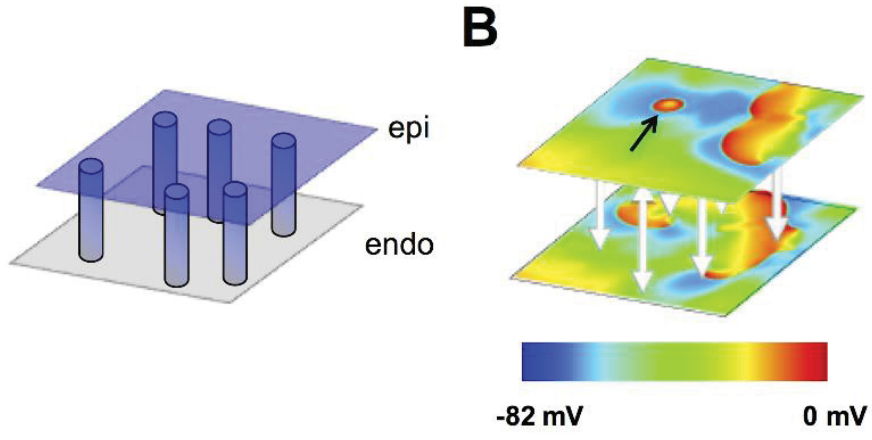

C

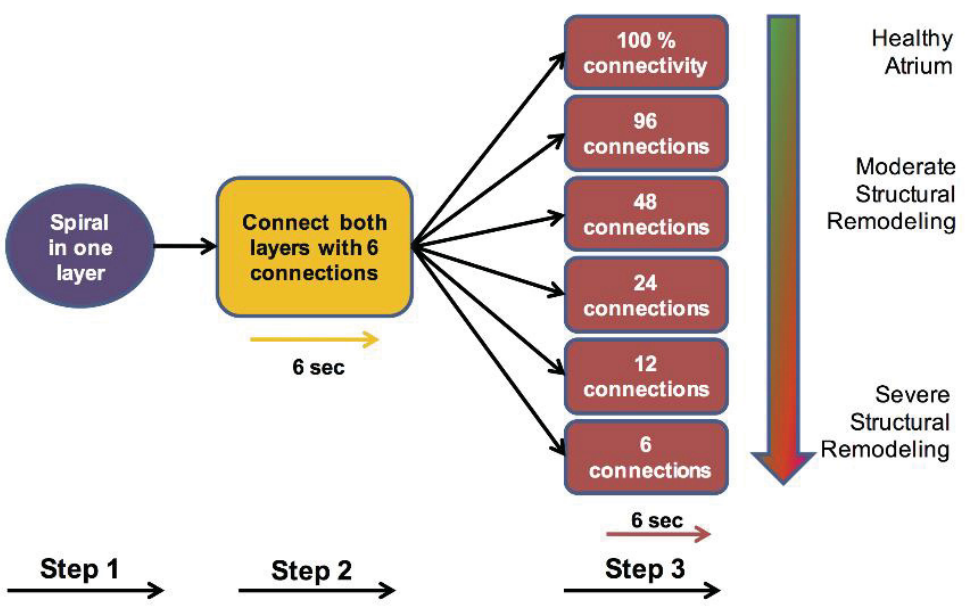

Figure 1. Dual layer model of the atrial wall $(A)$ Model structure with two layers (epi- and endocardium) and transmural connections (grey cylinders). (B) Dissociation of electrical activity between the two layers and transmural conduction resulting in a breakthrough wave (black arrow). (C) Simulations protocol (see methods section for details). 


\subsection{Analysis}

In all simulations the following parameters were analyzed (for algorithms used for these analyses see supplemental methods):

- Number of phase singularities (PSs)

- Phase singularity lifespan

- Breakthrough rates (BTRs)

- Breakthrough lifespans

- Number of fibrillation waves

- Wave front lifespans

- Atrial fibrillation cycle length (AFCL)

- Wave Front Conduction Velocity (WFCV)

- Excitable area (EA)

- Degree of endo-epicardial dyssynchrony

- Degree of endo-epicardial dissociation

- Atrial fibrillation duration

\section{Results}

Figure 2 shows representative simulations of AF driven by spiral waves for different degrees of endo-epicardial connectivity. Snapshots are shown for each $1000 \mathrm{~ms}$ (see online supplement for videos). When both layers are fully connected ( $100 \%$ connectivity, Figure $2 \mathrm{~A}$ ), the two layers synchronized within $50 \mathrm{~ms}$. No BTs were observed and the last depolarization waves disappeared after $2100 \mathrm{~ms}$.

In the examples with 96 and 48 connections (Figure $2 \mathrm{~B}-\mathrm{C}$ ), the two layers showed rapid synchronization by a high number of BTs. With increasing synchronization of the activity in the two layers, the number of waves, PSs, and BTR decreased. After $2000 \mathrm{~ms}$ the two layers showed synchronized activity and shortly thereafter, the episode terminated.

In the example with 24 connections (Figure 2D), dyssynchrony decreased between 1000 and $2000 \mathrm{~ms}$, which resulted in synchronized activity between 3000 and $4000 \mathrm{~ms}$ and termination of the episode at $4100 \mathrm{~ms}$. 
In the examples with 12 and 6 connections (Figure 2E-F), the initial rate of $\mathrm{BTs}$ is lower, no resynchronization of the two layers occurred, dyssynchrony of electrical activation between the two layers remained high, and the fibrillation patterns in both layers remained complex.

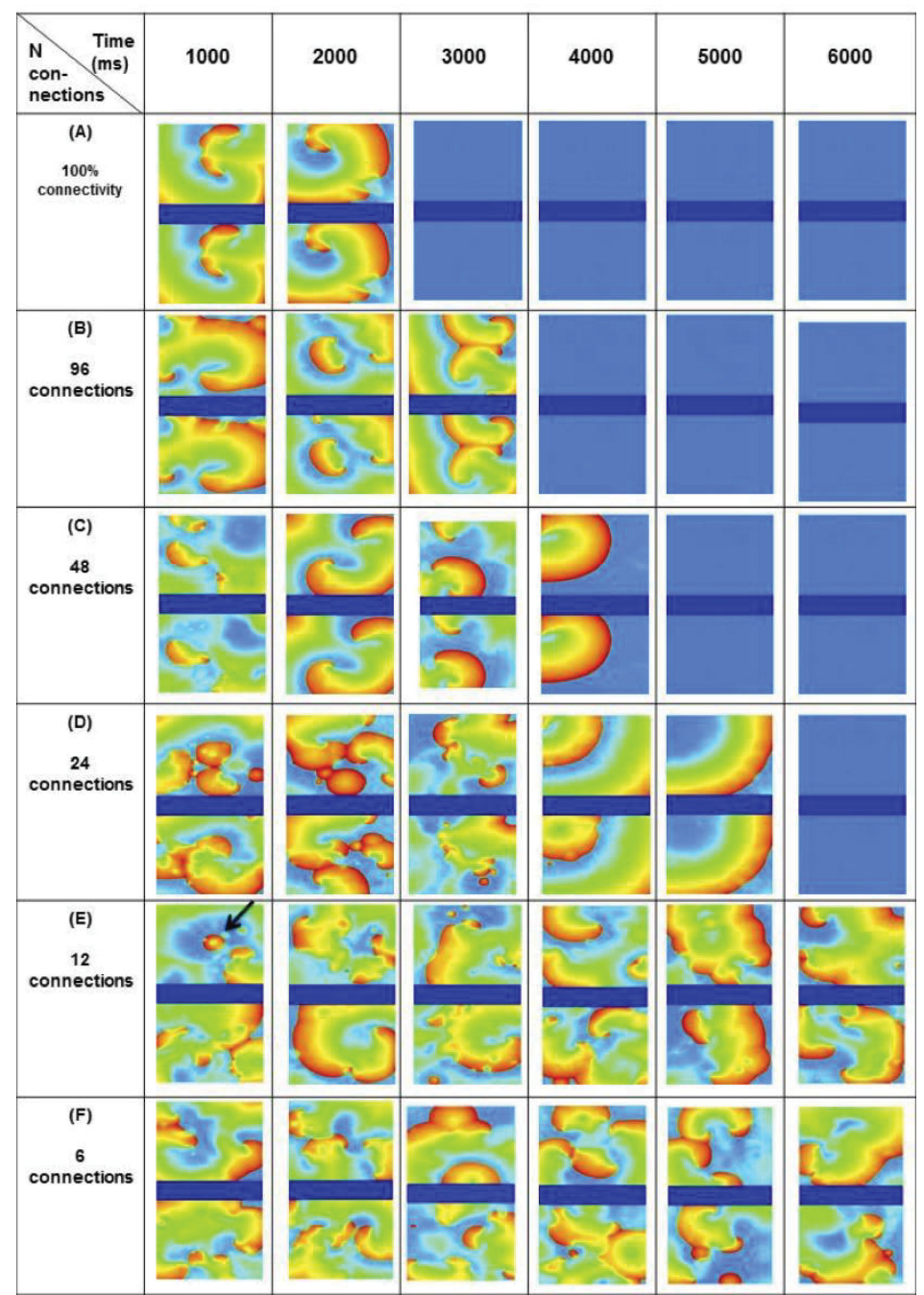

Figure 2. Colour-coded membrane potentials of representative spiral wave driven simulations with different degrees of endo-epicardial electrical coupling. The epicardial layer is shown in the top, the endocardial in the bottom panel. Snapshots are shown for every second of simulation time. 
In none of the simulations of AF driven by random ectopy, spiral wave initiation was observed. Propagation patterns remained organized and only consisted of either radial spread of ectopic waves or breakthroughs (Figure 3 ).

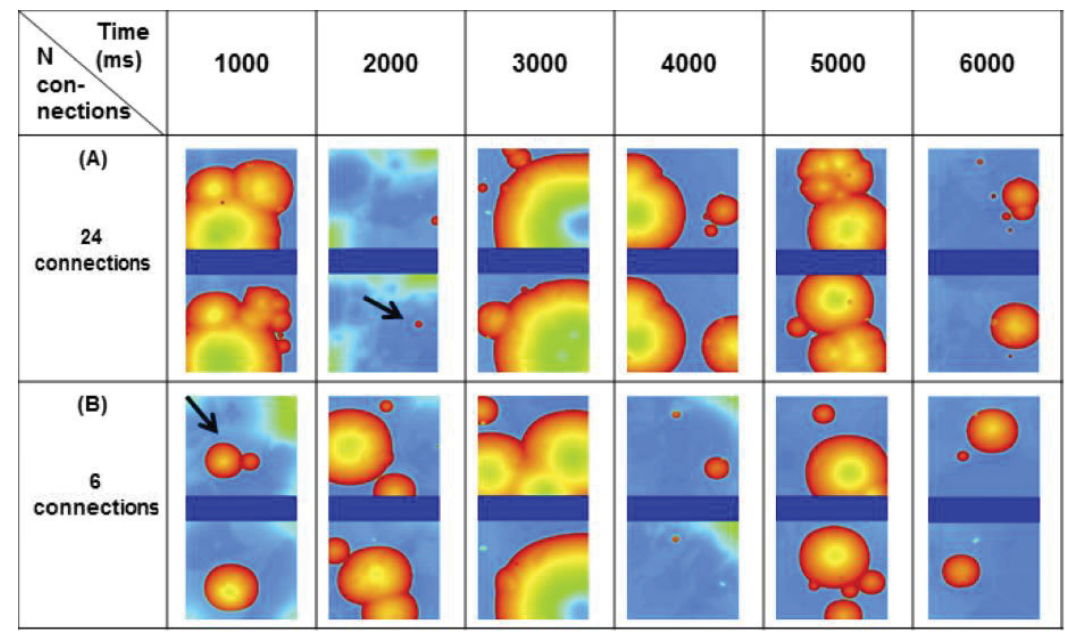

Figure 3. Colour-coded membrane potentials of representative of AF simulations driven by random ectopy with different degrees of endoepicardial electrical coupling. The epicardial layer is shown in the top, the endocardial in the bottom panel. Snapshots are shown for every second of simulation time. The black in the upper panel at $2000 \mathrm{~ms}$ is showing an ectopy. The arrow in the lower panel at $1000 \mathrm{~ms}$ is showing a BT.

\subsection{Parameters determining stability of AF}

Figure 4 shows the Kaplan-Meier curve of AF persistence in the set of 48 spiral waves driven simulations. With a progressive loss of connections, AF persistence strongly increased.

In order to study how loss of connectivity stabilizes AF, the effect of the number of connections on a variety of parameters describing the substrate for AF was studied. 


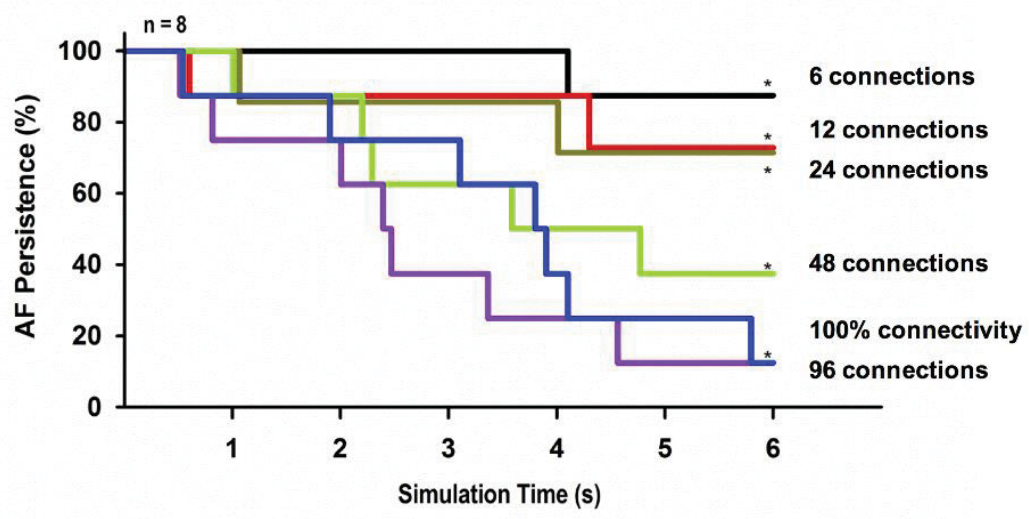

Figure 4. AF persistence. Kaplan-Meier curve showing atrial fibrillation persistence in different sets of simulations with $6,12,24,48,96$ connections, and $100 \%$ connectivity ( $n=8$ for all different sets of simulations).

Figure 5 shows the relation between the connectivity and number of phase singularities, phase singularity lifespan, breakthrough rates, breakthrough lifespan, number of waves and wave front lifespan, and electrophysiological parameters such as wave front conduction velocity (WFCV), AF cycle length (AFCL), excitable area (EA), breakthrough rate (BTR), and endo-epicardial dyssynchrony. The numbers of PSs at 24 and 96 connections were significantly higher compared to $100 \%$ connectivity. Consequently, PS lifespan at 24 connections and 96 connections were significantly shorter than at $100 \%$ connectivity $(p<0.05)$ (Figure $5 A-B$ ). As illustrated in Figure 5C-D, BTRs showed an increase with reduction of connections down to 24 connections, but with a further elimination of connections BTR decreased. No significant differences were found in BT lifespan between the groups.

The number of waves was significantly larger at 12, 24, 48, and 96 connections than at $100 \%$ connectivity (Figure 5E) while the wave front lifespans were shorter than at $100 \%$ connectivity (Figure 5F). There was no significant difference in AFCL, WFCV, and EA between simulations with different numbers of connections (see Figure 5G-I). Dyssynchrony progressively and strongly increased with the elimination of the endo-epicardial connections (Figure 5J). 

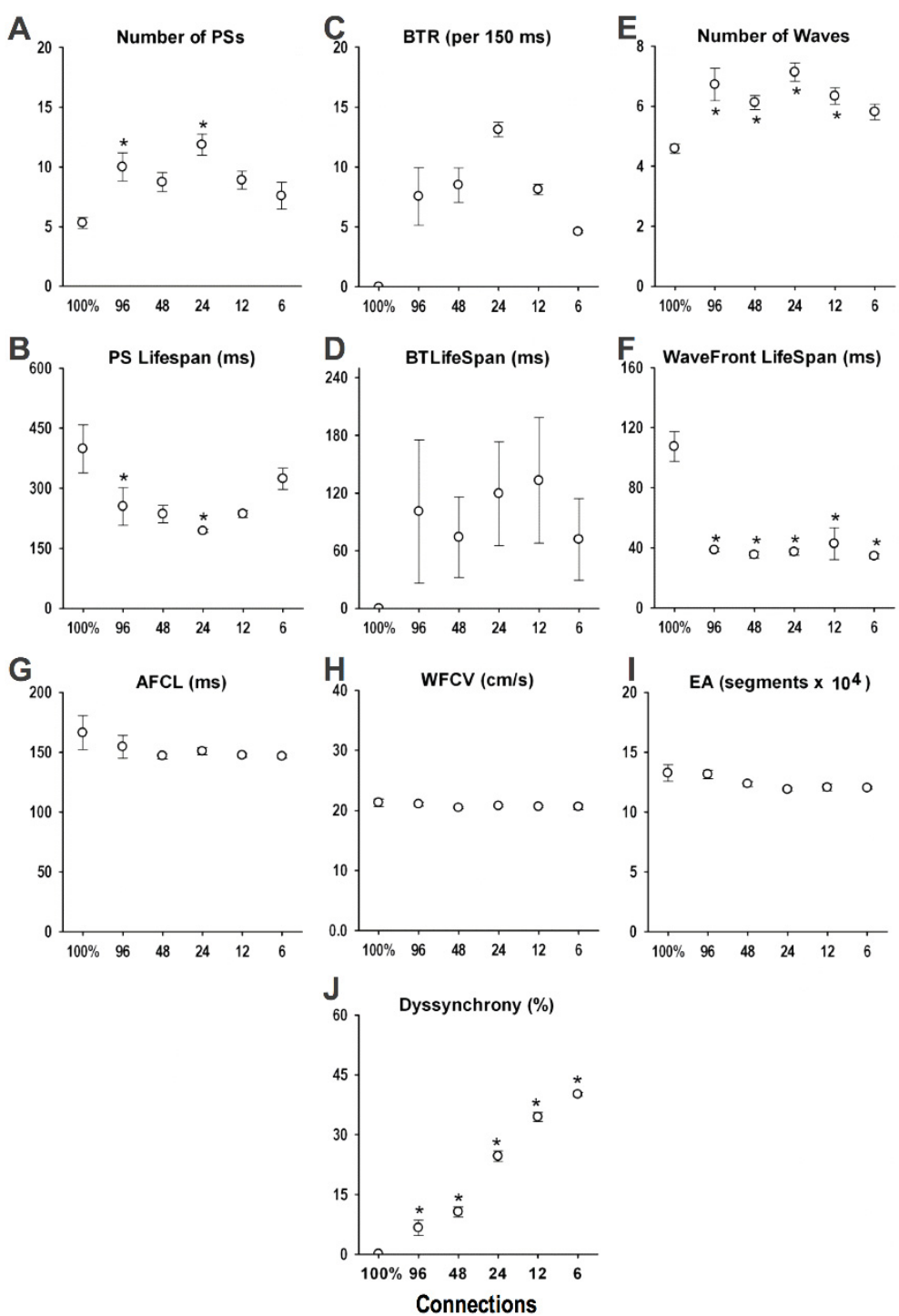

Figure 5. Electrophysiological parameters as a function of the degree of connectivity. Averages for the whole simulation period $(6000 \mathrm{~ms})$ are shown for all parameters. A) Average number of PSs; B) PS lifespan; C) Average breakthrough rates; D) Breakthrough lifespan; E) Average number of waves; F) Wave front lifespan. G) Average AF cycle lengths; H) Average wave front conduction velocity; I) Average excitable area; J) Average degree of dyssynchrony. 
The same parameters were compared between all episodes that were sustained and those that were not sustained irrespective of the number of connections (Figure 6). This analysis was performed to identify which parameters were associated with AF stability independently from the number of connections.

A

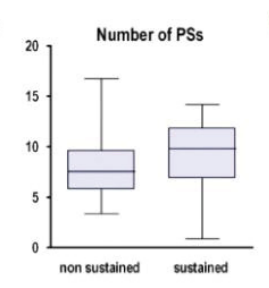

B
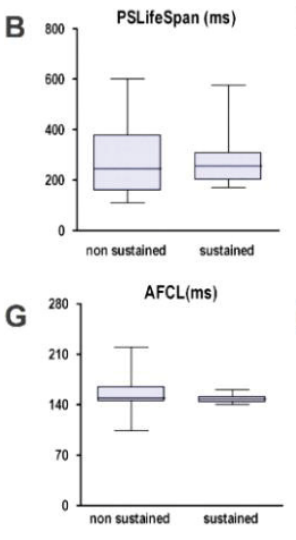
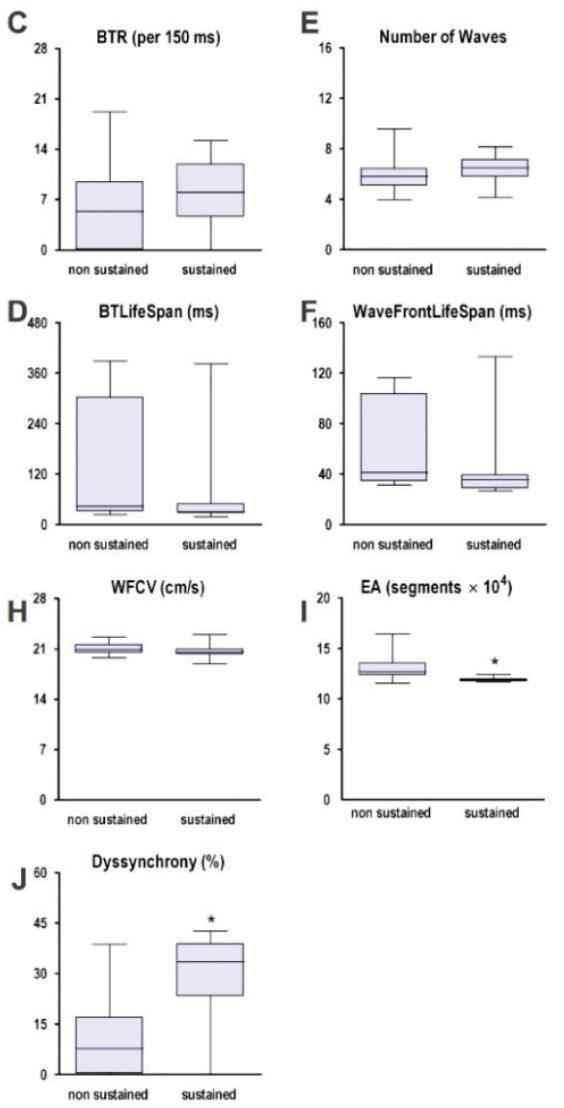

Figure 6. Differences in electrophysiological parameters between nonsustained $(<6 \mathrm{sec})$ and sustained AF episodes. All parameters were averaged for the entire durations the of AF episodes. A) Average number of PSs; B) PS lifespan; C) Average breakthrough rates; D) Breakthrough lifespan; E)Average number of waves; F) Wave front lifespan; G) Average AF cycle lengths; H) Average wave front conduction velocity; I) Average excitable area; J) Average degree of dyssynchrony. 
In panels $\mathrm{A}$ and $\mathrm{B}$, the number of PSs and PS lifespans are shown. There were no significant differences between the two groups in these parameters. There were neither differences in BTR and BT lifespan (panel $C$ and $D$ ) nor in the number of waves or wave front lifespan (panel E and F). There were no differences in AFCL and WFCV, but EA was slightly but significantly lower in sustained versus non-sustained AF (panels $G, H$, and I). The most striking difference was that the degree of dyssynchrony was much higher in the group with sustained AF compared to non-sustained AF (group J).

In order to investigate which parameter is the strongest determinant of AF duration, we used multilevel logistic regression analysis among measured parameters. Three parameters were significantly correlated to AF stability (see Table 1).

Table 1. Association between electrophysiological parameters and AF stability determined by multilevel logistic regression analysis.

\section{EP Parameters $\quad P$ Values Coefficients}

\begin{tabular}{lll}
\hline PSs & 0.639 & 0.0519 \\
PS Lifespan & 0.270 & 0.0053 \\
BTs & 0.369 & -0.1644 \\
BT Lifespan & 0.244 & -0.0035 \\
Waves & 0.720 & 0.1157 \\
Wave Front Lifespan & 0.957 & 0.0015 \\
EA & $0.027^{*}$ & $-0.0013^{*}$ \\
AFCL & 0.245 & -0.0348 \\
WFCV & 0.2070 & -65.441 \\
Dyssynchrony & $0.004^{*}$ & $0.1315^{*}$ \\
Connections & $0.01^{*}$ & $-.0628^{*}$ \\
\hline
\end{tabular}


Out of these significant predictive parameters, dyssynchrony was by far the strongest determinant of AF persistence in this set of simulations.

In random ectopy driven simulations, AF was sustained by pacing so that a study on AF stability was not applicable.

\subsection{Relation between dyssynchrony and breakthrough rate}

In Figure 7A, dyssynchrony (blue line), BT rate (red line), and BT rate during the first $50 \mathrm{~ms}$ after adding the connection points are plotted for spiral wave driven AF. The BT rate during the first $50 \mathrm{~ms}$ after adding the connection points is of interest because in this short time interval the two layers have not yet synchronized and the BT rate largely depends on the number of available endoepicardial connections. This 'initial' BT rate, decreases with the number of connections (black line). However, the overall BT rate for the whole AF episode showed a biphasic relation to the number of connections, increasing from 100\% connectivity down to 24 connections, but decreasing for 12 and 6 connections. 


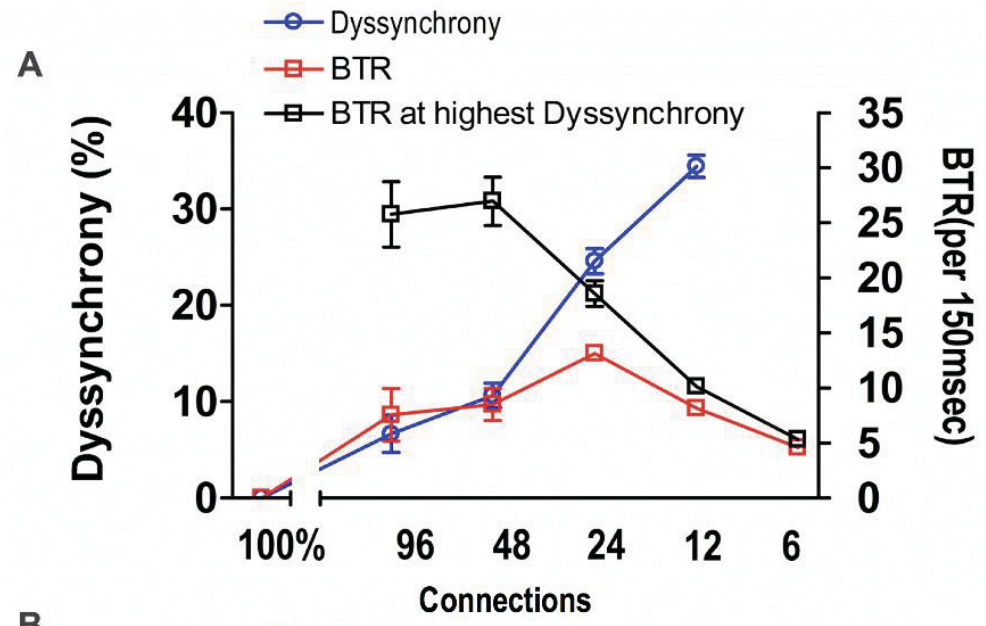

(3)

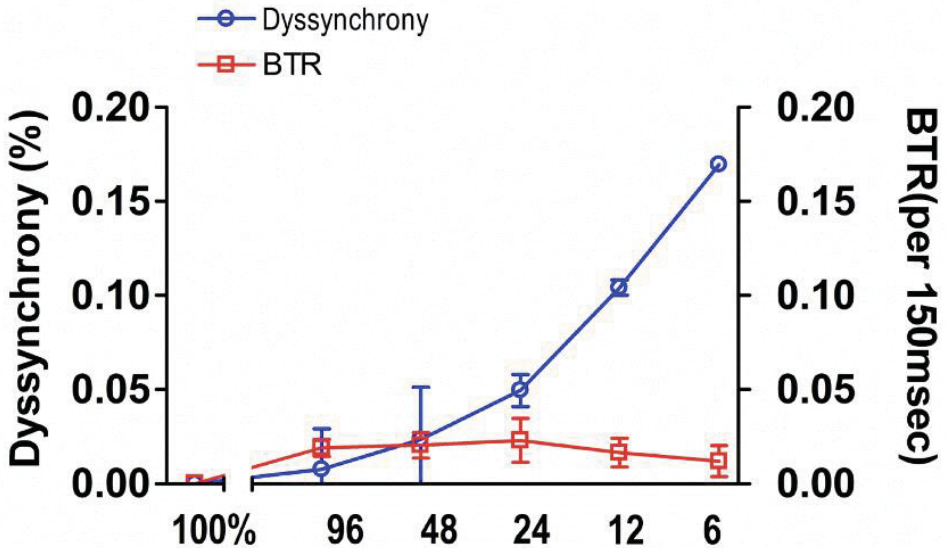

Figure 7. A) Relation of BTR and dyssynchrony to the degree of epiendo connectivity in spiral wave driven simulations. Average percentage of dyssynchrony(blue) over the entire period of each simulation. Average BTR over the entire duration of the episodes (red) and within the first $50 \mathrm{~ms}$ (black, highest degree of dyssynchrony). B) Relation of BTR and dyssynchrony to the degree of epi-endo connectivity in simulations of AF driven by random ectopy. Average percentage of dyssynchrony (blue) over the entire period of each simulation. Average BTR over the entire duration of the episodes (red). 
In Figure 7B, dyssynchrony (blue line) and BT rate (red line) extracted from random pacing simulations are illustrated. The overall BT rate of the whole AF episode showed a biphasic relation to the number of connections, increasing from $100 \%$ connectivity down to 24 connections, but decreasing for 12 and 6 connections. This pattern was similar to that observed in our spiral wave driven simulations.

\subsection{Comparison of experimental data and simulation data}

In Figure 8, the BT rate (as percentage of all waves) is plotted against the degree of dissociation for experimental ${ }^{10,19}$ and simulation data extracted from spiral waves driving simulations. For this analysis, dissociation was calculated both for modeling data and experimental data as previously described. ${ }^{10,19}$

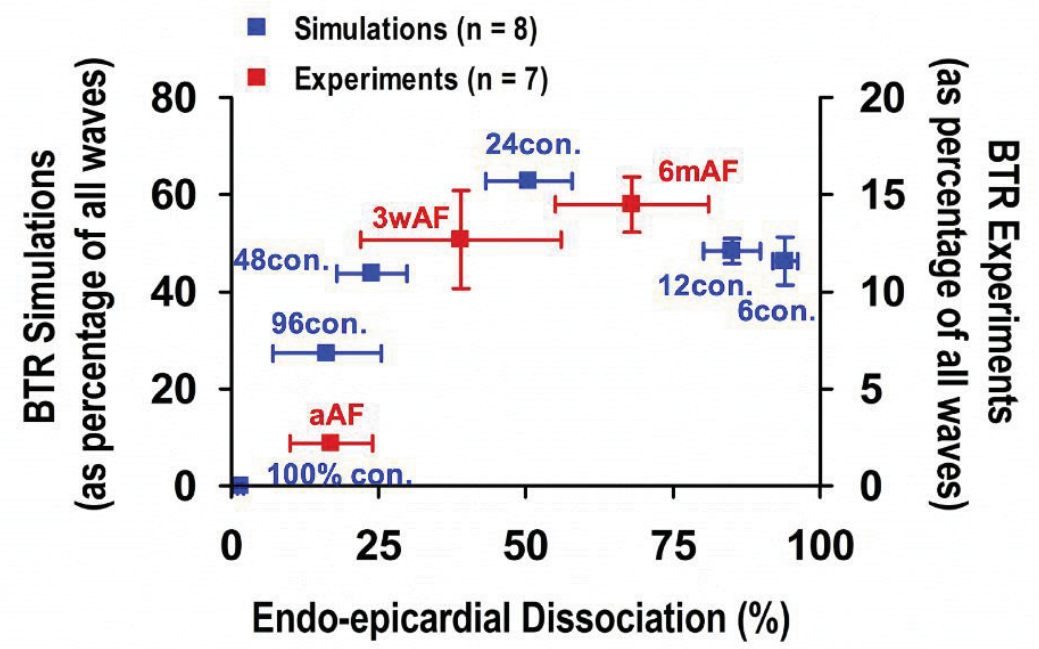

Figure 8. Comparison of modeling data with experimental results. Breakthrough rates (as percentage of all waves) versus the degree of dissociation observed in experimental (red squares) and simulation studies (blue squares). Abbreviations: con: number of connections in simulation studies; aAF: goats in acute AF; 3mAF: goats after 3 weeks of AF; $6 \mathrm{mAF}$ : goats after 6 months of AF. 
Experimental data (open squares) includes data derived from goats in acute $A F(a A F, n=7), 3$ weeks of $A F(3 w A F, n=7)$, and 6 months of $A F(6 m A F, n=7)$ and were taken from a previous study. ${ }^{19}$ As illustrated in this figure, the degree of endo-epicardial dissociation increased with AF duration. To some extent of dissociation, increase in degree of dissociation leads to increase in BT both in experimental ( $3 \mathrm{WAF}$ ) and modeling (24 connections). Beyond that, even with huge increase in dissociation only slight increase in BT rate in experimental (6mAF) and decrease in modeling (12 connections) result was observed. Overall, although there was not point to point match between experimental and modeling results but patterns were similar.

\section{Discussion}

The mechanisms contributing to an increase in stability of AF are not fully understood. Electrical remodeling, electrical uncoupling between muscle bundles, and heterogeneity of action potential duration all together have been hypothesized to increase stability of AF. ${ }^{2}, 20$ More specifically, disruption of electrical side-to-side connections between muscle bundles occurs as a consequence of structural alterations in the atrial wall. ${ }^{1}$ This loss of connectivity between muscle bundles leads to an enhanced likelihood of conduction block, the number of fibrillation waves, and AF stability. ${ }^{12}$ Disruption of electrical side-to-side connections does not only occur within the epicardial layer but most likely also between the epicardial layer and the endocardial bundle network. ${ }^{10,} 11$ This uncoupling between the epi- and endocardial layers of the atrial wall - at a first glance - should lead to less transmural conduction events resulting in a lower incidence of breakthrough during AF. However, in goats as well as in patients the incidence of breakthroughs has been shown to be much higher in persistent than in acute forms of the arrhythmia. 2, 8, 10, 19 The present study was undertaken to clarify how loss of endo-epicardial connectivity might result in more transmural conduction and higher breakthrough rates. We hypothesized that disruption of endo-epicardial connections increases the dissociation of electrical activity 
between the epicardial layer and the endocardial muscle bundles. As a prerequisite for transmural conduction, increased endoepicardial dissociation of electrical activity will enhance transmural conduction and result in a higher breakthrough rate despite lower availability of endo-epicardial connections and, as a consequence, an increase in AF duration.

\subsection{Model Structure}

There are two groups of atrial models with respect to geometrical properties: surface models which treat the atria as two dimensional sheet folded into the shape of the atria (with two main cavities, atrial appendages, and connections between the two atria at the septal rings $)^{22-24}$ and the true $3 \mathrm{D}$ volumetric models. Volumetric models go into greater detail in modeling the atrial wall including such properties as complex anatomy of endocardium bundle networks ${ }^{25-28}$ and fiber orientations in atrium. ${ }^{29-31}$ Several mechanisms were investigated using volumetric models such as the effect of ectopies, AP heterogeneities, fibrosis and meandering of phase singularities on fibrillatory propagation patterns and AF stability. A few recent studies also investigated the effect of atrial wall thickness of fibrillatory patterns and on AF maintenance. ${ }^{9,21}$

While all these models were useful to answer many important questions about mechanisms contributing to AF stability none of them considered endo-epicardial dyssynchrony, transmural conduction, and their effect on AF stability.

The present study was designed to investigate the relationship between breakthrough incidences, endo-epicardial dyssynchrony and AF stability. We chose a dual layer model instead of volumetric 3D model because: (i) The exact location and distribution of muscle bundles providing endo-epicardial electrical connections is very difficult to determine experimentally. Any simulation implementing a detailed anatomy of such endo-epicardial connection points would suggest a level of accuracy that can currently not be achieved even with advanced imaging techniques. (ii) We aimed to exclusively focus on the quantitative determinants of endoepicardial dissociation and conduction without any confounding 
effect of complex 3D geometry, which due to scope and complexity could not be addressed in this study. Due to all above mentioned reasons and to avoid complexities that make us unable to investigate the dynamics of transmural conduction property we chose a simplified geometry proof-of-principle model.

\subsection{Effect of transmural uncoupling on dyssynchrony and breakthroughs}

The novelty of our model is that it describes truly threedimensional (transmural) conduction between the endocardial and the epicardial layer ${ }^{13}$ and by doing so introduces a new type of simulated fibrillation waves, i.e. breakthroughs. In this study we demonstrated that a decrease in the degree of coupling between the endocardium and the epicardium increased the degree of dyssynchrony and in contrast increased the incidence of transmural conduction until a certain degree of connectivity. Our results demonstrate that this increase can be explained by the enhanced dyssynchrony between the two layers. If both opposing layers at a connection point are either excited or non-excited, no transmural conduction occurs. Only in the presence of dyssynchronous electrical activity, fibrillation waves can propagate along endo-epicardial connections. As a result, in the presence of a high number of connections the breakthrough rate is low because the degree of endo-epicardial dissociation is low, despite the fact that the number of available endo-epicardial connections is high. Interestingly, increasing the extent of uncoupling to a larger degree (fewer than 24 connections) showed an opposite effect. The breakthrough rate decreased with further decrease in the number of connections while the degree of dyssynchrony increased. To explain this phenomenon, we measured the breakthrough rate during a short period just after introducing more connections (i.e. the start of step 3 in figure 2), when the layers have not yet synchronized. During this period we calculated the number of BTs during highest achieved degree of dyssynchrony. Under these circumstances, we observed that the breakthrough rate strongly and monotonously declined with a reduction of the number of endo- 
epicardial connections, just as expected based on the simple reasoning that reduced number of available electrical pathways should reduce the occurrence of endo-epicardial conduction. The results indicate that under these modeling conditions the $B T$ rate not only depends on the degree of dyssynchrony, but also is limited by the actual number of endo-epicardial connection points. In case only a few connections exist between the two layers, breakthroughs will occur less frequently, because of the low number of available endo-epicardial connections, despite a high degree of endoepicardial dissociation.

We also showed that our conclusions are transferable to circumstances under which AF is maintained by other mechanisms. We tested the proposed relation between endo-epicardial connections, endo-epicardial dissociation and breakthrough rate in a modification of the model in which the arrhythmia is driven by multiple ectopic foci. This mechanism has been shown to perpetuate AF under specific circumstances, such as vagal nerve stimulation. ${ }^{33}$ Under these conditions, we identified exactly the same pattern in the quantitative relation between number of endoepicardial connection points, endo-epicardial dissociation and breakthrough rate.

\subsection{Effect of transmural uncoupling on AF stability}

In this study, we demonstrate that a decrease in transmural coupling increases AF episode stability in spiral waves driven simulations. Not all of the important determinants of AF stability such as AF cycle length and conduction velocity were altered by the different degrees of endo-epicardial coupling. Only the excitable area was slightly but significantly affected. Importantly, all differences observed between small and large degrees of uncoupling were related to three-dimensional conduction. AF duration increased with decreasing number of connections and we identified dyssynchrony as the strongest predictor for AF stability. Taken together, these findings clearly suggest that the threedimensional character of the conduction pattern in itself is an important determinant of the stability of the arrhythmia. 


\subsection{Relation between experimental results and the model data}

The phenomenon of endo-epicardial dissociation of electrical activity has been experimentally studied by several research groups. Schuessler et al. ${ }^{32}$ were the first to reveal endo-epicardial dyssynchrony of activation during acetylcholine-induced AF in canine right atria. Gray et al. and $\mathrm{Wu}$ et al. also investigated the three-dimensional structure of the atria and its effect on activation sequences during atrial fibrillation and also observed transmural conduction incidences. ${ }^{6,7}$ Everett et al. compared epicardial direct contact electrograms with non-contact mapping endocardial electrograms and found larger endo-epicardial activation time differences in a rapid atrial pacing model than in control animals. ${ }^{34}$ Also the elegant study of Yamazaki et al. demonstrated the occurrence of endo-epicardial dissociation during AF in sheep with atrial dilatation. ${ }^{9}$ Recently, Eckstein et al. demonstrated that the degree of endo-epicardial dissociation increases during the course of $A F{ }^{10,} 11$ The mechanism of this increasing dissociation of electrical activity was not related to endo-epicardial dispersion of refractoriness but rather to loss of endo-epicardial coupling between muscle bundles. Very recently, we demonstrated that loss of continuity in the epicardial layer and anatomical rearrangement of atrial muscle bundles also contribute to the increase in endoepicardial dissociation during the development of persistent AF. ${ }^{5,18}$ Our modeling data to some extent showed similar pattern with several experimental and clinical studies published during the past years. Both in patients and in goats, enhanced rates of breakthrough have been demonstrated in persistent AF compared to acute $\mathrm{AF}^{8}{ }^{8}$ The relationship between endo-epicardial dissociation and breakthroughs rate found in the simulation study is comparable but not identical with the one reported by Eckstein et al. ${ }^{10,19}$ The difference in BT rate between experimental and simulation finding could be because of the much greater complexity of the true atrial anatomy compared to our simplified model. Nevertheless, despite differences in absolute values, the nature of the relation is qualitatively similar between the model and experimental results. 
Both studies show that at low degrees of endo-epicardial dissociation the breakthrough rate is low. At moderate levels of endo-epicardial dissociations the breakthrough rate increases. Interestingly, a further increase in endo-epicardial dissociation (above 50\%) only leads to small increase in breakthrough rates in experimental study but decrease in modeling study. Thus, in the range of physiological degrees of endo-epicardial uncoupling, the degree of endo-epicardial dissociation rather than the number of available endo-epicardial electrical connections is the limiting factor for the breakthrough rate.

\subsection{Clinical relevance}

Our study strongly supports the importance of a loss of connectivity between epicardial and endocardium that is an integral part of the process of structural remodeling. The resulting increase in endo-epicardial dissociation of electrical activity increases AF complexity by increasing the tortuosity of fibrillatory conduction, allowing more fibrillation waves to coexist in the atrium, thereby increasing AF stability. ${ }^{35}$ For ablation strategies, this supports the notion that the lesions, whether created by an endocardial or epicardial approach, should be truly transmural. Upstream therapy would be beneficial if it could prevent the loss of connectivity between the epicardial layer and the endocardial bundle network, e.g. by inhibiting fibrosis. This would in effect reduce the area available to fibrillation waves and should therefore strongly reduce AF stability.

\section{Study limitations}

Our simplified dual-layer model should be considered as a proofof-principle model. This model does not reflect complex structure of the atrium with pulmonary veins, valves, heterogeneity in ionic membrane currents, and variability in atrial wall thickness. Despite these limitations, we have shown that our model is well-suited to investigate the relation between of endo-epicardial dissociation and 
breakthroughs on AF complexity and stability. How additional complexities in the anatomy of the atrial wall affect endo-epicardial dissociation and transmural conduction should be addressed in future studies. 


\section{References:}

1. Spach MS, Dolber PC. Relating extracellular potentials and their derivatives to anisotropic propagation at a microscopic level in human cardiac muscle. Evidence for electrical uncoupling of sideto-side fiber connections with increasing age. Circ Res 1986;58:356-371.

2. Verheule S, Tuyls E, van Hunnik A, Kuiper M, Schotten U, Allessie M. Fibrillatory conduction in the atrial free walls of goats in persistent and permanent atrial fibrillation. Circ Arrhythm Electrophysiol 2010;3:590-599.

3. Schotten U, Verheule S, Kirchhof P, Goette A. Pathophysiological mechanisms of atrial fibrillation: a translational appraisal. Physiological reviews 2011;91:265-325.

4. Verheule S, Eckstein J, Linz D, Maesen B, Bidar E, Gharaviri A, et al. Role of endo-epicardial dissociation of electrical activity and transmural conduction in the development of persistent atrial fibrillation. Prog. Biophysics and molecular biology 2014;15:173185.

5. Maesen B, Zeemering S, Afonso C, Eckstein J, Burton RA, van Hunnik $A$, et al. Rearrangement of atrial bundle architecture and consequent changes in anisotropy of conduction constitute the 3-dimensional substrate for atrial fibrillation. Circ Arrhythm Electrophysiol 2013;6:967-975.

6. Gray RA, Pertsov AM, Jalife J. Incomplete reentry and epicardial breakthrough patterns during atrial fibrillation in the sheep heart. Circulation 1996;94:2649-2661.

7. Wu TJ, Yashima M, Xie FG, Athill CA, Kim YH, Fishbein MC, et al. Role of pectinate muscle bundles in the generation and maintenance of intra-atrial reentry - Potential implications for the mechanism of conversion between atrial fibrillation and atrial flutter. Circ Res 24 1998;83:448-462.

8. de Groot NM, Houben RP, Smeets JL, Boersma E, Schotten U, Schalij MJ, et al. Electropathological substrate of longstanding persistent atrial fibrillation in patients with structural heart disease: epicardial breakthrough. Circulation 2010;122:16741682.

9. Yamazaki M, Mironov S, Taravant C, Brec J, Vaquero LM, Bandaru $\mathrm{K}$, et al. Heterogeneous atrial wall thickness and stretch 
promote scroll waves anchoring during atrial fibrillation. Cardiovasc Res 2012;94:48-57.

10. Eckstein J, Maesen B, Linz D, Zeemering S, van Hunnik A, Verheule $S$, et al. Time course and mechanisms of endoepicardial electrical dissociation during atrial fibrillation in the goat. Cardiovasc Res 2011;89:816-824.

11. Eckstein J, Zeemering S, Linz D, Maesen B, Verheule S, van Hunnik A, et al. Transmural Conduction is the Predominant Mechanism of Breakthrough during Atrial Fibrillation: Evidence from Simultaneous Endo-epicardial High Density Activation Mapping. Circ Arrhythm Electrophysiol 2013; 6(2):334-41.

12. Allessie MA, de Groot NM, Houben RP, Schotten U, Boersma E, Smeets JL, et al. Electropathological substrate of long-standing persistent atrial fibrillation in patients with structural heart disease: longitudinal dissociation. Circ Arrhythm Electrophysiol 2010;3:606-615.

13. Gharaviri A, Verheule S, Eckstein J,Potse M, Kuijpers N, Schotten U. A computer Model of Endo-Epicardial Dissociation and Transmural Conduction During Atrial Fibrillation. Europace 2012; 14 Suppl 5:v10-v16.

14. Courtemanche M, Ramirez RJ, Nattel S. Ionic mechanisms underlying human atrial action potential properties: insights from a mathematical model. The American journal of physiology 1998;275:H301-321.

15. Leon LJ, Roberge FA, Vinet A. Simulation of two-dimensional anisotropic cardiac reentry: effects of the wavelength on the reentry characteristics. Ann Biomed engineering 1994;22:592609.

16. Kuijpers $\mathrm{NH}$, Keldermann $\mathrm{RH}$, Arts T, Hilbers PA. Computer simulations of successful defibrillation in decoupled and nonuniform cardiac tissue. Europace 2005;7 Suppl 2:166-177.

17. Kuijpers NH, Keldermann RH, ten Eikelder HM, Arts T, Hilbers PA. The role of the hyperpolarization-activated inward current If in arrhythmogenesis: a computer model study. IEEE Trans Biomed Eng 2006;53:1499-1511.

18. Verheule S, Tuyls E, Gharaviri A, Hulsmans S, van Hunnik A, Kuiper $\mathrm{M}$, et al. Loss of continuity in the thin epicardial layer due to endomysial fibrosis increases the complexity of atrial fibrillatoryconduction.CircArrhythmElectrophysiol 2013;6(1):20211. 
19. Eckstein J. Three-Dimensional Substrate of Atrial Fibrillation in the Goat: A Simultaneous Endo-epicardial Mapping Study. Maastricht: Physiology Department, Maastricht University; 2012.

20. Berenfeld O, Zaitsev AV, Mironov SF, Pertsov AM, Jalife J. Frequency-dependent breakdown of wave propagation into fibrillatory conduction across the pectinate muscle network in the isolated sheep right atrium. Circ Res 14 2002;90:1173-1180.

21. Kuijpers NH, Potse M, van Dam PM, ten Eikelder HM, Verheule S, Prinzen FW, et al. Mechanoelectrical coupling enhances initiation and affects perpetuation of atrial fibrillation during acute atrial dilation. Heart Rhythm 2011;8:429-436.

22. Uldry L, Virag N, Lindemans F, Vesin JM, Kappenberger L. Atrial septal pacing for the termination of atrial fibrillation: study in a biophysical model of human atria. Europace 2012;14 Suppl 5:v112-v120.

23. Haissaguerre M, Lim KT, Jacquemet V, Rotter M, Dang L, Hocini $M$, et al. Atrial fibrillatory cycle length: computer simulation and potential clinical importance. Europace 2007;9 Suppl 6:vi64-70.

24. Ruchat P, Dang L, Schlaepfer J, Virag N, von Segesser LK, Kappenberger L. Use of a biophysical model of atrial fibrillation in the interpretation of the outcome of surgical ablation procedures. Eur J Cardiothorac Surg 2007;32:90-95.

25. Aslanidi OV, Colman MA, Stott J, Dobrzynski H, Boyett MR, Holden AV, et al. 3D virtual human atria: A computational platform for studying clinical atrial fibrillation. Prog Biophys Mol Biol 2011;107:156-168.

26. Colman MA, Aslanidi OV, Kharche S, Boyett MR, Garratt CJ, Hancox JC, et al. Pro-arrhythmogenic Effects of Atrial Fibrillation Induced Electrical Remodelling- Insights from 3D Virtual Human Atria. J Physiol 2013;591:4249-72.

27. Vigmond EJ, Ruckdeschel R, Trayanova N. Reentry in a morphologically realistic atrial model. J Cardiovasc Electrophysiol 2001;12:1046-1054.

28. Gong YF, Xie F, Stein KM, Garfinkel A, Culianu CA, Lerman BB, et al. Mechanism underlying initiation of paroxysmal atrial flutter/atrial fibrillation by ectopic foci - A simulation study. Circulation 2007;115:2094-2102.

29. Zhao J, Butters TD, Zhang H, Pullan AJ, LeGrice IJ, Sands GB, et al. An image-based model of atrial muscular architecture: effects of structural anisotropy on electrical activation. Circ Arrhythm Electrophysiol 2012;5:361-370. 
30. Krueger MW, Severi S, Rhode K, Genovesi S, Weber FM, Vincenti $A$, et al. Alterations of atrial electrophysiology related to hemodialysis session: insights from a multiscale computer model. J Electrocardiol 2011;44:176-183.

31. Kharche S, Seemann G, Margetts L, Leng J, Holden AV, Zhang HG. Simulation of clinical electrophysiology in 3D human atria: a high-performance computing and high-performance visualization application. Concurr Comp-Pract E 2008;20:1317-1328.

32. Schuessler RB, Kawamoto T, Hand DE, Mitsuno M, Bromberg BI, Cox JL, et al. Simultaneous epicardial and endocardial activation sequence mapping in the isolated canine right atrium. Circulation 1993;88:250-263.

33. Lee S, Sahadevan J, Khrestian CM, Durand DM, Waldo AL. High density mapping of atrial fibrillation during vagal nerve stimulation in the canine heart: restudying the Moe hypothesis. J Cardiovasc Electrophysiology 2013;24(3):328-335

34. Everett TH 4th, Wilson EE, Hulley GS, Olgin JE. Transmural characteristics of atrial fibrillation in canine models of structural and electrical atrial remodeling assessed by simultaneous epicardial and endocardial mapping. Heart Rhythm 2010;7:506517.

35. Verheule S, Eckstein J, Linz D, Maesen B, Bidar E, Gharaviri A, et al. Role of endo-epicardial dissociation of electrical activity and transmural conduction in the development of persistent atrial fibrillation. Prog Biophys Mol Biol. 2014;115(2-3):173-85. 


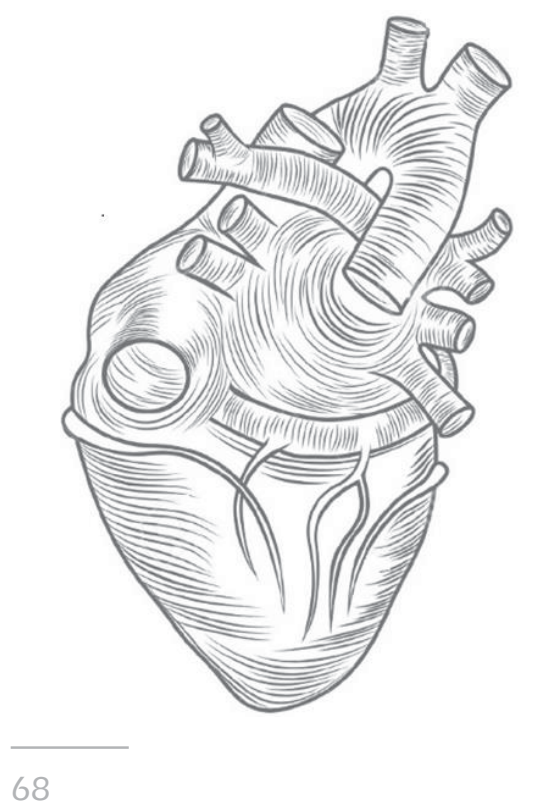




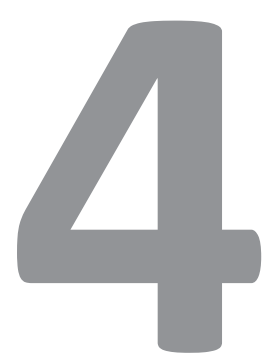

Loss of continuity in the thin epicardial layer due to endomysial fibrosis increases the complexity of atrial fibrillatory conduction

Sander Verheule

Els Tuyls

Ali Gharaviri

Sarah Hulsmans

Arne van Hunnik

Marion Kuiper

Jan Serroyen

Stef Zeemering

Nico H.L. Kuijpers

Ulrich Schotten

Published in Circ Arrhythm

Electrophysiol. 2013 Feb;6(1):202-11. doi: 10.1161/CIRCEP.112.975144.

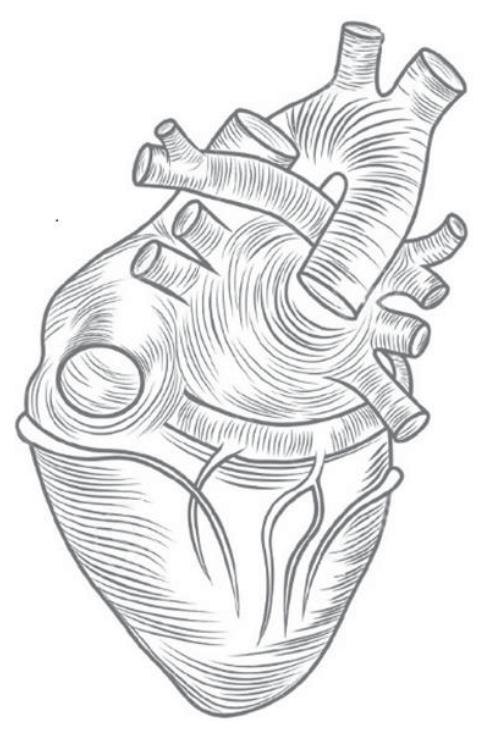




\section{Abstract}

\section{Background:}

The transition from persistent to permanent atrial fibrillation (AF) is associated with increased complexity of fibrillatory conduction. We have investigated the spatial distribution of fibrillation waves and structural alterations in the atrial free walls in a goat model of AF.

\section{Methods and Results:}

AF was maintained for 3 weeks (ST, persistent AF) or 6 months (LT, permanent AF). Fibrillation patterns were assessed with epicardial mapping. The origin of fibrillation waves and sites of conduction abnormalities were more homogeneously distributed in LT than in ST goats. Histologically, the total area fraction occupied by fibrous tissue and the degree of perimysial fibrosis (separation between myocyte bundles) were not significantly different between groups. However, endomysial fibrosis (distance between myocytes within bundles) was significantly larger in LT goats, particularly in the outer millimeter of the atria. By contrast, myocyte diameters were larger in LT goats throughout the atrial walls. High-resolution optical mapping showed that epicardial wavefront expansion was slower and more anisotropic in LT than in ST goats. Finally, a mathematical model of a simplified atrial architecture confirmed the potential impact of epicardial endomysial fibrosis on AF complexity.

\section{Conclusions:}

Altered propagation after 6 months of $\mathrm{AF}$ is consistent with homogeneous structural remodelling in the outer millimeter of the atria. Loss of continuity of the epicardial layer due to endomysial fibrosis may reduce its synchronizing effect, thereby increasing the complexity of fibrillatory conduction pathways. The exact distribution of fibrosis may be more important for the occurrence of conduction disturbances than the overall quantity. 


\section{Introduction}

In many patients suffering from atrial fibrillation (AF), the arrhythmia becomes more entrenched over months to years. ${ }^{1}$ This progression in AF stability is mimicked in several animal models. In goats with artificially maintained AF, AF episode duration gradually increases. $^{2}$ Even after AF has become sustained (i.e. not selfterminating) in this model, AF stability still gradually increases over the ensuing months. Sinus rhythm can be restored by pharmacological cardioversion after 3-4 weeks of AF (persistent AF), but not after 4-6 months of AF (permanent AF)., ${ }^{4}$ We have previously demonstrated that the complexity of fibrillation patterns in the atrial free walls is higher in permanent AF than in persistent $\mathrm{AF}^{4}$. This increased complexity, characterized by a higher number of smaller wavefronts, is thought to contribute to higher AF stability. ${ }^{5}$ A higher complexity of fibrillation patterns in the atrial free walls has also been observed in patients with chronic AF compared to patients with acute or paroxysmal AF ${ }^{6-9}$ In paroxysmal AF patients, high-frequency activity in specific atrial areas, such as the pulmonary veins, may contribute disproportionately by triggering AF episodes. ${ }^{10}$ However, as atrial remodeling progresses and $\mathrm{AF}$ becomes more stable as a consequence, the entire atrial myocardium can contribute to the substrate for AF., 9,11

A major candidate mechanism for causing the changes in fibrillatory conduction and the concomitant increase in AF stability is structural remodelling, which develops over the same slow time course of months. ${ }^{12}$ In the goat model of AF, structural remodeling includes fibrosis and myocyte hypertrophy, ${ }^{4,12}$ both of which can affect atrial conduction. ${ }^{13,14}$ The type (i.e. reactive vs. replacement) and exact distribution of fibrous tissue over the atrial walls are pertinent to its effect on atrial conduction, but have not been studied extensively. ${ }^{15}$ The atria have a complex anatomy, with a thin epicardial layer overlying an extensive endocardial trabecular network. ${ }^{16}$ We have recently reported that during the progression from acute to persistent and permanent AF, the degree of dissociation in fibrillation waves between the epicardium and the endocardial bundle network increases. ${ }^{17}$ Goats with acutely induced 
AF showed $18 \%$ dissociation of electrical activity between epi- and endocardium, while dissociation had increased to $40 \%$ after 3 weeks of AF and to $70 \%$ after 6 months of AF. As a result, the atria become a more '3-dimensional' substrate for AF, which is also reflected by an increased incidence of epicardial breakthrough waves, both in this goat model $^{4}$ and in AF patients. ${ }^{9}$

To elucidate the structural substrate for $A F$, we have analyzed the spatial distribution of fibrillation waves and structural changes in goats with 3 weeks (short term, ST) and 6 months (long term, LT) AF. We report that myocyte hypertrophy occurs throughout the atrial wall in LT, but that endomysial fibrosis occurs predominantly in the outer millimeter of the epicardium. Atrial epicardial wavefront propagation, showed ubiquitous alterations throughout the free walls that are compatible with transverse uncoupling of myocyte strands due to endomysial fibrosis. Although the total amount of fibrosis is much smaller than in some other animal models of AF, we propose that the impact of this particular type of fibrosis on atrial conduction may be considerable, thus providing an interesting target for specific treatment strategies.

\section{Methods}

\subsection{Animal model}

AF was maintained by burst pacing for 3 weeks $(S T, n=10)$ or 6 months $(\mathrm{LT}, \mathrm{n}=7)$. In open-chest follow-up experiments, propagation patterns in the atrial free walls were mapped during AF and the spatial distribution of peripheral waves and epicardial breakthroughs (waves appearing at the periphery and within the central part of the recording area, respectively) and slow conduction were analyzed (see supplement).

\subsection{Histological analysis}

After the electrophysiological measurements, complete atria of 5 ST and 5 LT goats were sectioned and stained with toluidine-blue 
in order to distinguish myocytes (dark blue) from connective tissue

(light blue), see supplement.

\subsection{Optical mapping}

Optical mapping was performed in perfused hearts from separate groups of ST $(n=8)$ and LT $(n=6)$ goats. A $100 \times 100$ pixel camera was used to image the fluorescence of the voltage-sensitive dye di-4ANEPPS in $2 \times 2 \mathrm{~cm}$ areas of the LA free wall during stimulation within the field of view (see supplement).

\subsection{Mathematical model}

To investigate the effect of reduced transverse electrical coupling in the epicardial layer on AF complexity and the incidence of epicardial breakthroughs, a dual-layer mathematical model was used (see supplement).

\subsection{Statistical analysis}

Histological data were analyzed using a linear mixed model (SAS v9.2, SAS Institute). Myocyte diameters were normally distributed, but intermyocyte and interbundle distances were log-transformed to correct right-skewness. Morisita indices, which were not normally distributed, were tested with a Kruskall-Wallis test and a Dunn's post-hoc test. Time series of wavefront spread were analyzed using a general linear regression model for repeated measures (SPSS v20, IBM Inc.). $P<0.05$ was considered statistically significant.

\section{Results}

\subsection{Spatial characteristics of fibrillation patterns}

We have previously reported that in both atria of LT goats, compared to ST goats, conduction block is more frequent and the 
number of peripheral waves and epicardial breakthroughs (EBs) is larger, leading to a more complex, dissociated fibrillation pattern, while the AFCL is not significantly different. ${ }^{4}$ Here, we have studied the spatial distribution pattern of fibrillation waves in the same goats. Figure $1 \mathrm{~A}$ depicts the spatial pattern of EB waves. Compared to ST goats, the total number of EBs was higher in LT goats, but the spatial pattern was more homogeneous. The Morisita index, which reflects the degree of clustering, tended to be lower in the LT RA and was significantly lower in the LT LA, compared to ST atria. As apparent in figure 1A, EBs tended to occur more frequently at some electrode sites than at others, particularly in ST goats. However, the average interval between EBs at a certain site was much longer than the AFCL in both atria of both groups (ST LA: $1334 \pm 444 \mathrm{~ms}$, ST RA: $1328 \pm 133 \mathrm{~ms}$, LT LA: $1199 \pm 130 \mathrm{~ms}$, LT RA: $891 \pm 90 \mathrm{~ms}$ ). Thus, sites where epicardial breakthrough originated did not behave as localized high-frequency sources.

Similarly, the number of peripheral waves was higher in LT goats, but distributed more homogeneously around the periphery (figure 1B), leading to a lower Morisita index (significant for the LA). The incidence of long conduction times $(>8 \mathrm{~ms})$ between neighboring electrodes was also higher in LT atria, but again with a more dispersed pattern, leading to a lower Morisita index (significant for the LA). A similar analysis is shown in the data supplement for the distribution of AFCLs (supplemental figure 1A). There however, the pattern in LT goats showed more clustering, translating into a higher Morisita index for both atria, due to regions within the recorded area with a lower activation frequency. The average AFCL was not systematically higher in either atrium: the RA had a shorter AFCL than the LA in 5 out of 10 ST goats and in 5 out of 7 LT goats (supplemental figure 1B). 
A
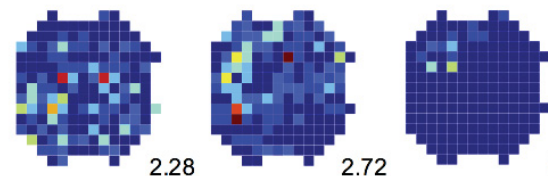

Morisita Index

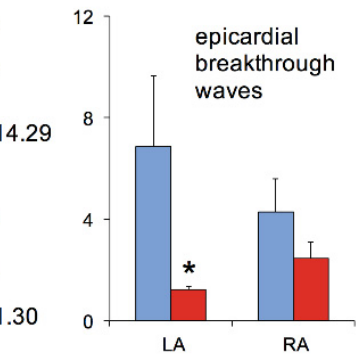

LT
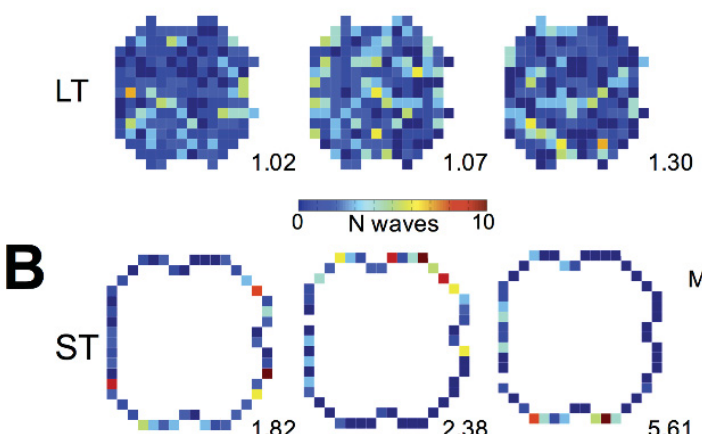

Morisita Index
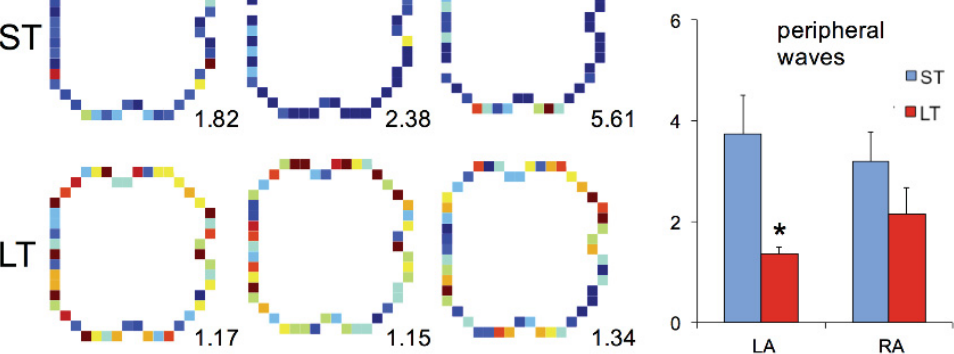

$0 \quad \mathrm{~N}$ waves 10

C
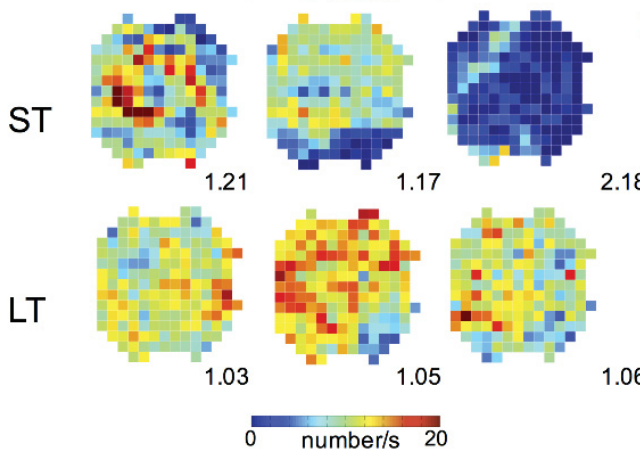

Morisita Index

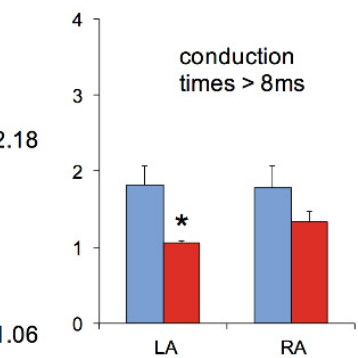

Figure 1. Spatial pattern of fibrillatory conduction. A, B and C show examples of 3 ST and 3 LT LA, along with their Morisita indices (a measure for spatial clustering, mean \pm s.e.m., $* p<0.01$ ). Distribution of A) epicardial breakthroughs, B) peripheral waves and C) slow conduction (conduction times $>8 \mathrm{~ms}$ between neighboring electrodes). The spatial dispersion in all three parameters (right panels) tended to be lower in LT goats, with significant differences in the LA. 


\subsection{Relevant aspects of the atrial anatomy}

The atrial walls have a complex anatomy (endocardial aspect in figure 2A). The anterior parts possess an extensive network of trabeculae underlying the epicardial layer, which is as thin as $1 \mathrm{~mm}$ in many places (figure $2 \mathrm{~B}$ ). Figure $2 \mathrm{C}$ and animated stacks of aligned atrial slices (see supplement) illustrate that endocardial bundles give off branches to the epicardial layer at various sites along their length. These branches to the epicardium are often orientated perpendicularly to the underlying trabeculae. In this sense, the thin epicardial layer is formed by the final branches of the underlying trabecular network. Within small areas of the free wall such as in figure $2 \mathrm{C}$, a variety of epicardial fiber orientations was visible.

\subsection{Distribution of structural remodeling due to long term AF}

To help understand the difference in fibrillation pattern between ST and LT goats, we have studied the distribution of fibrous tissue and myocyte size. No large areas of scar tissue were apparent in any of the atria investigated. Moreover, the overall amount of intramyocardial fibrous tissue was not different between ST and LT goats in either atrium (supplemental data figure 2). In figure $3 \mathrm{~A}$, we have quantified the number of larger interruptions $(>0.5 \mathrm{~mm})$ in the epicardial layer. The number of interruptions was higher in the RA than in the LA, but did not differ between the ST and LT groups in either atrium. To further investigate to distribution of fibrous tissue, we distinguished between perimysial fibrosis (fibrous tissue surrounding bundles of myocytes) and endomysial fibrosis (fibrous tissue separating individual myocytes within bundles). These parameters were quantified in relation to the distance to the epicardium. Perimysial fibrosis, quantified as the distance between muscle bundles, showed a wide distribution throughout the atrial wall, without significant differences between the RA and LA and between the ST and LT groups (figure 3B). 


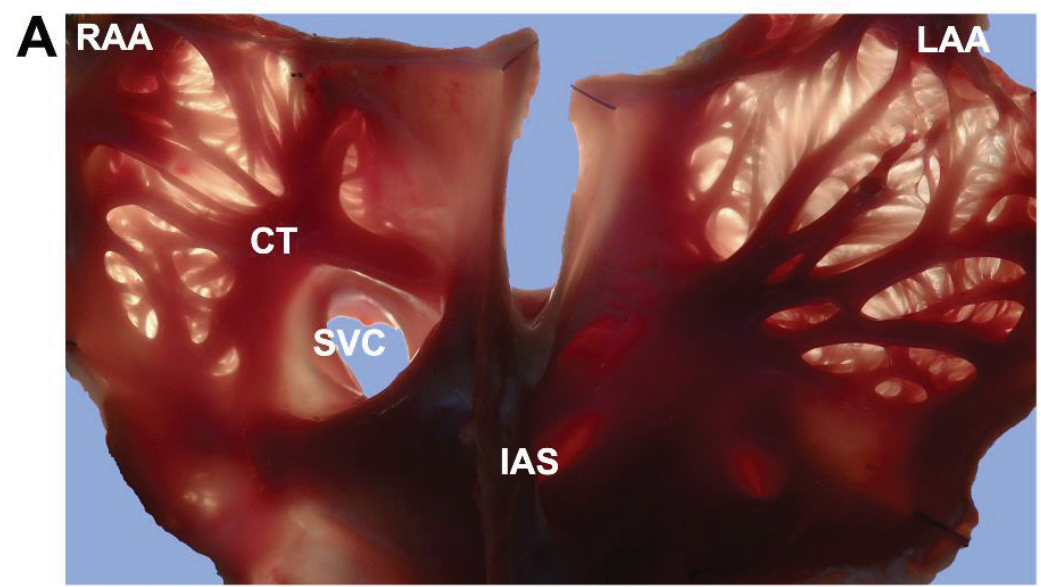

B

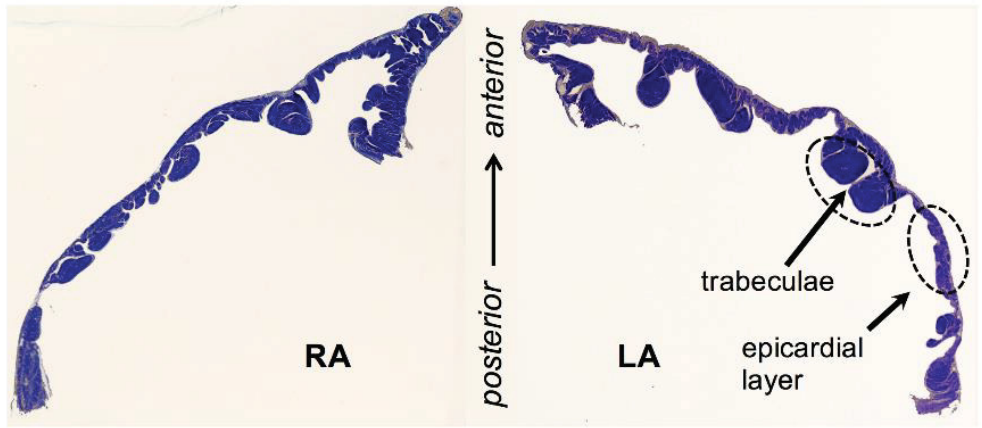

C

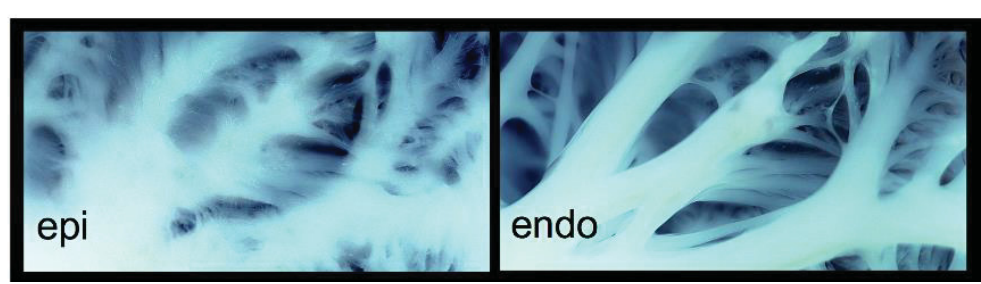

Figure 2. Anatomical structure of the atria. A) Excised and backilluminated atrial preparation shows the extensive trabecular network underlying the thin epicardial layer (CT: crista terminalis, SVC: superior vena cava, IAS: inter-atrial septum, RAA and LAA: RA and LA appendage). B) Sections from the RA and LA, illustrating endocardial trabeculae connecting to the epicardial layer. C) Epi-and endocardial view of part of the LA free wall (detail of preparation in A, colors inverted for clarity). The epicardial layer is formed by the final branches of the endocardial trabecular network, with epicardial fibers often running at right angles to larger trabeculae. 
A
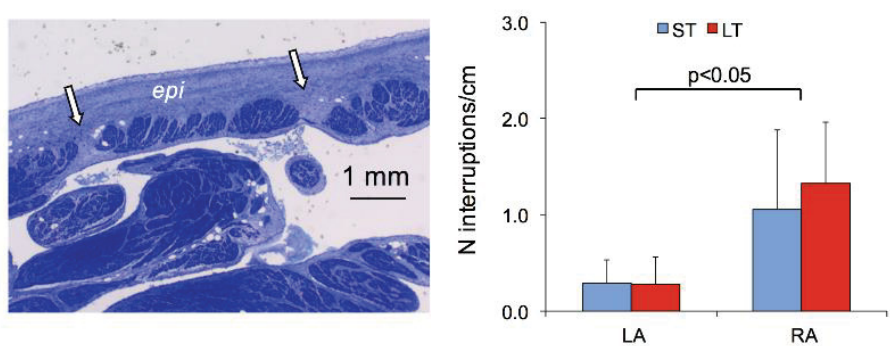

B
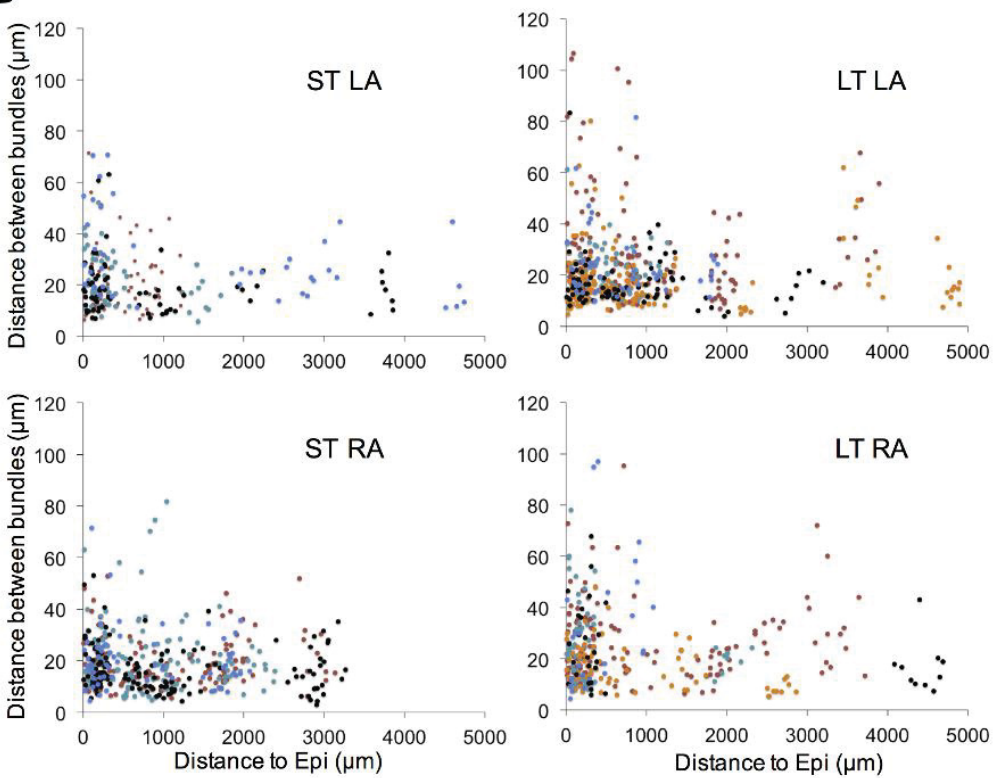

Figure 3. Transmural distribution of perimysial fibrosis. A) The incidence of interruptions $>0.5 \mathrm{~mm}$ in the epicardial layer (examples indicated by arrows) did not differ between the ST and LT groups (mean \pm s.d.). B) Distance between myocyte bundles (perimysial fibrosis) as a function of the distance to the epicardium. Different colors of dots represent data from different goats. Perimysial fibrosis was homogeneously distributed throughout the wall and was not significantly different between the atria and between ST and LT goats.

The degree of endomysial fibrosis, quantified as the distance between myocytes within bundles, was low throughout the atrial wall in the ST LA and RA (figure 4B), as evidenced by the close 
apposition of myocytes in figure $4 \mathrm{~A}$. In the $\mathrm{LT}$ atria, there was a higher degree of endomysial fibrosis, visible as larger inter-myocyte distances in figure 4A. Interestingly, this larger degree of endomysial fibrosis was much more pronounced in the outer millimeter of the LT LA and RA than in deeper layers (figure 4B). In LT goats, the average myocyte diameter was significantly larger than in ST goats (figure 5A). However, in contrast to the pattern of endomysial fibrosis, the myocyte diameter was homogeneously larger throughout the atrial wall in LT goats (figure 5B).

Figure 6A (left panel) illustrates that the increase in endomysial fibrosis in LT goats was more pronounced in the thin epicardial layer than in the underlying trabeculae. The middle panel in figure $6 \mathrm{~A}$ depicts that, even in areas where the epicardial layer was relatively thick, increased endomysial fibrosis was still predominantly observed in the outer $1 \mathrm{~mm}$. The bar chart in the right panel provides average intermyocyte distances for endocardial trabeculae and for the outer millimeter of the epicardial layer in regions where the epicardial layer was relatively thin $(\leq 1 \mathrm{~mm})$ and relatively thick $(>1 \mathrm{~mm})$. The degree of endomysial fibrosis was more pronounced in the outer millimeter of the atrial wall, regardless of the local wall thickness, and less pronounced in the trabeculae.

Figure 6B summarizes the degree of peri- and endomysial fibrosis and myocytes diameter in total, in the outer millimeter of the atrial wall and in deeper layers ( $>1 \mathrm{~mm}$ from the epicardium). In deeper layers, myocyte diameter was increased in LT goats to the same degree as in the outer millimeter. By contrast, the degree of endomysial fibrosis was more pronounced in the outer millimeter than in deeper layers. 

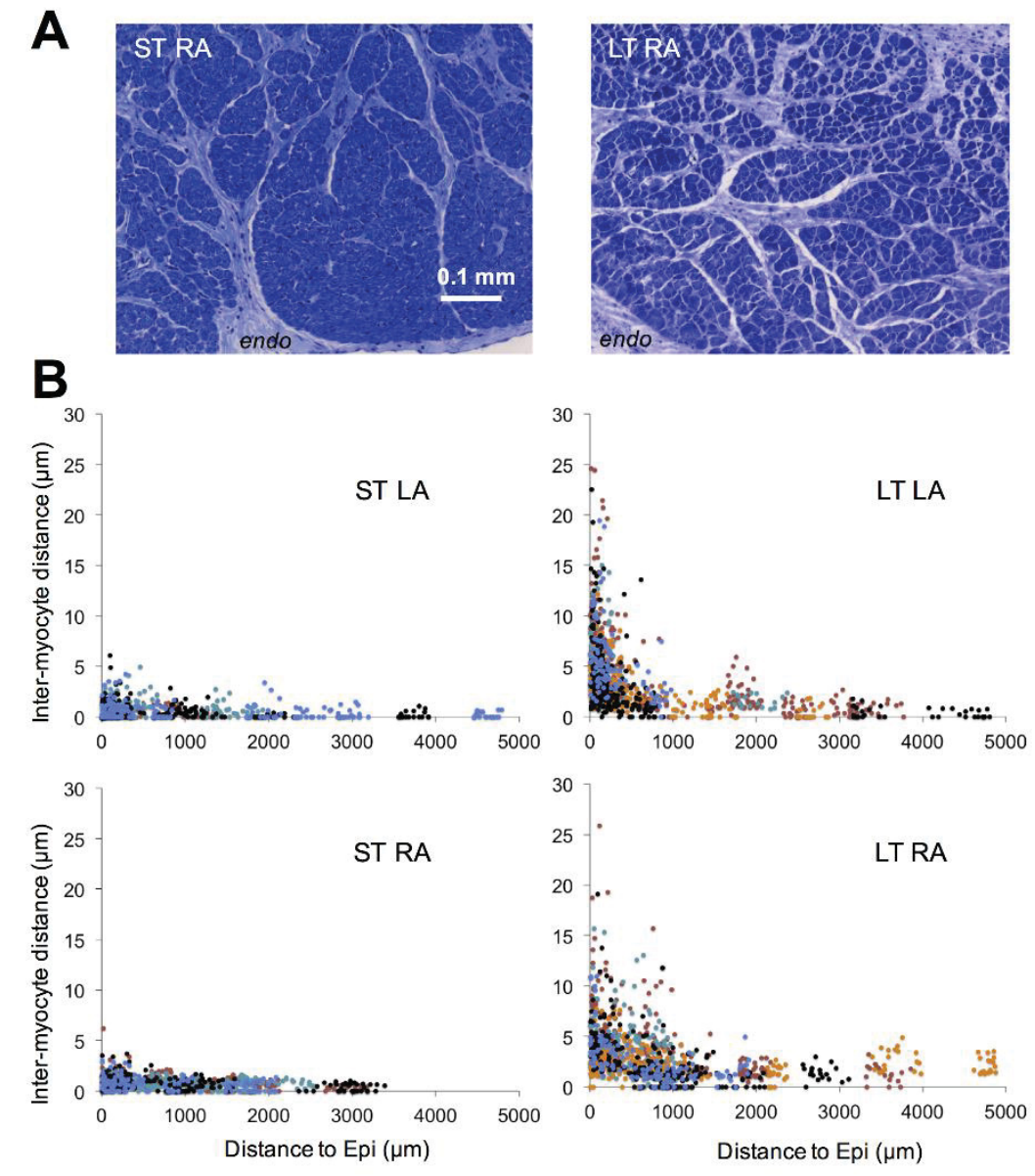

Figure 4. Transmural distribution of endomysial fibrosis. A) Examples of the RA myocardium close to the epicardium in an ST and LT goat, with larger myocyte-to-myocyte distances in the LT goat. B) Intermyocyte distance (endomysial fibrosis) as a function of the distance to the epicardium. Compared to the ST LA and RA, large intermyocyte distances were observed in the outer millimeter of the LT LA and RA. 

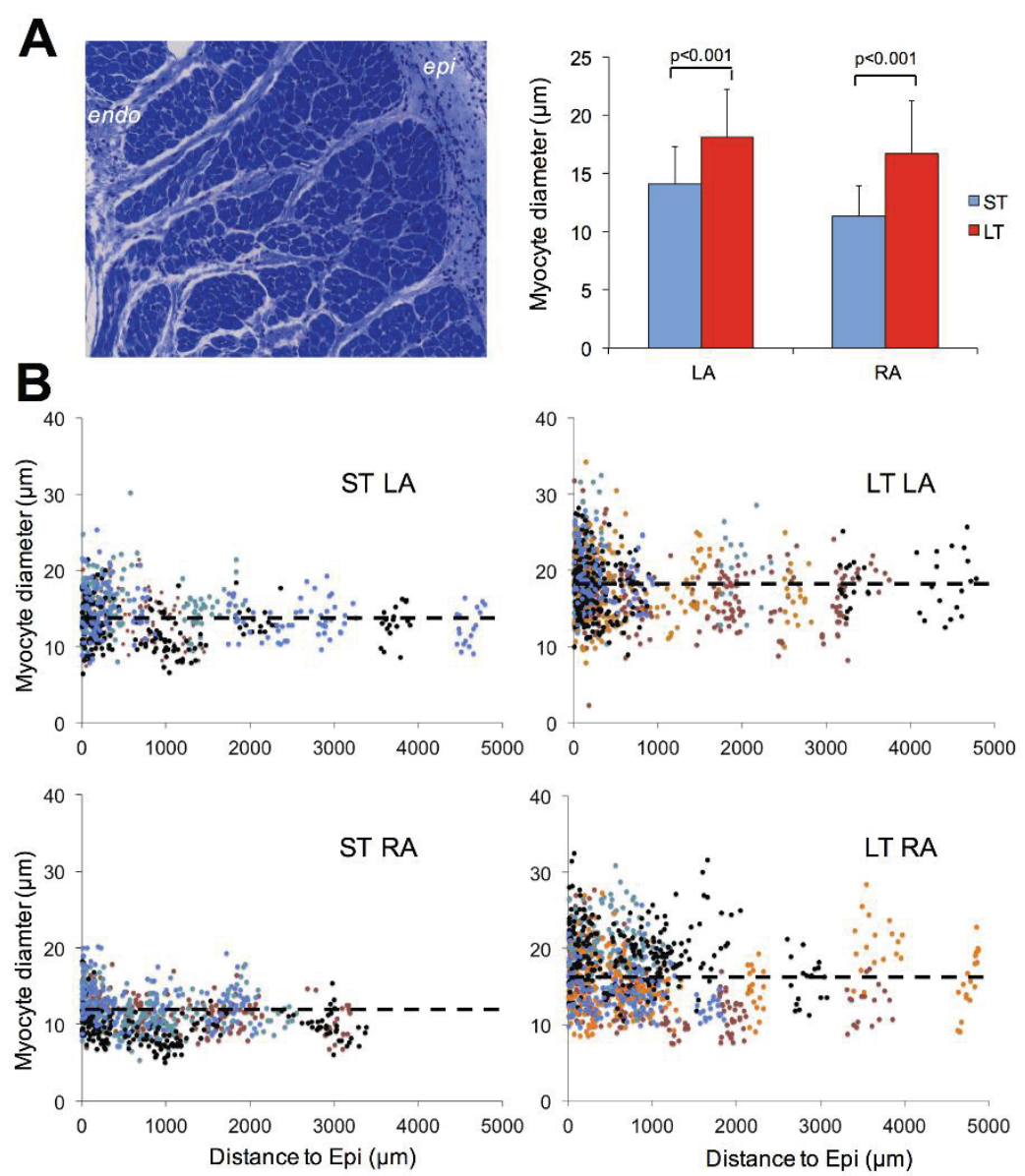

Figure 5. Transmural distribution of cardiomyocyte hypertrophy. A) The diameter of transversely sectioned myocytes was determined at the level of the nucleus. Average myocyte diameters (mean $\pm s d$ ).were significantly larger in LT goats than in ST goats in both atria. B). Myocyte diameter as a function of the distance to the epicardium (dotted lines indicate average diameters). In the LT LA and RA, myocyte diameters were larger throughout the atrial walls compared to the ST LA and RA. 

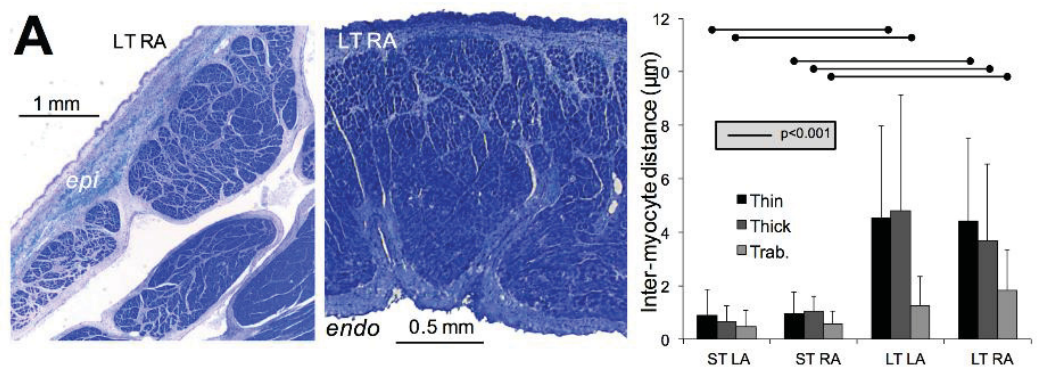

\begin{tabular}{|c|c|c|c|c|c|c|c|c|c|c|c|c|}
\hline 2 & \multicolumn{4}{|l|}{ total } & \multicolumn{4}{|c|}{ outer millimeter } & \multicolumn{4}{|c|}{ deeper layers } \\
\hline & \multicolumn{2}{|l|}{ LA } & \multicolumn{2}{|l|}{ RA } & \multicolumn{2}{|l|}{ LA } & \multicolumn{2}{|l|}{ RA } & \multicolumn{2}{|l|}{ LA } & \multicolumn{2}{|l|}{ RA } \\
\hline & ST & LT & ST & LT & ST & LT & ST & LT & ST & LT & ST & LT \\
\hline $\begin{array}{l}\text { distance } \\
\text { between } \\
\text { bundles }\end{array}$ & $\begin{array}{l}25.2 \\
\pm 21.2\end{array}$ & $\begin{array}{l}19.8 \\
\pm 11.6\end{array}$ & $\begin{array}{l}19.0 \\
\pm 10.9\end{array}$ & $\begin{array}{l}24.1 \\
\pm 15.5\end{array}$ & $\begin{array}{l}27.8 \\
\pm 25.0\end{array}$ & $\begin{array}{l}19.1 \\
\pm 10.7\end{array}$ & $\begin{array}{l}19.3 \\
\pm 11.3\end{array}$ & $\begin{array}{l}25.5 \\
\pm 16.6\end{array}$ & $\begin{array}{l}20.4 \\
\pm 9.0\end{array}$ & $\begin{array}{l}21.4 \\
\pm 13.2\end{array}$ & $\begin{array}{l}18.4 \\
\pm 10.1\end{array}$ & $\begin{array}{l}20.4 \\
\pm 11.7\end{array}$ \\
\hline $\begin{array}{l}\text { distance } \\
\text { between } \\
\text { myocytes }\end{array}$ & $\begin{array}{l}0.69 \\
\pm 0.81\end{array}$ & $\begin{array}{l}3.74 \\
\pm 3.64\end{array}$ & $\begin{array}{l}0.82 \\
\pm 0.63\end{array}$ & $\begin{array}{l}3.13 \\
\pm 2.74\end{array}$ & $\begin{array}{l}0.81 \\
\pm 0.86\end{array}$ & $\begin{array}{l}4.57 \\
\pm 3.76\end{array}$ & $\begin{array}{l}0.96 \\
\pm 0.68\end{array}$ & $\begin{array}{l}3.72 \\
\pm 2.93\end{array}$ & $\begin{array}{l}0.39 \\
\pm 0.57\end{array}$ & $\begin{array}{l}1.06 \\
\pm 0.97\end{array}$ & $\begin{array}{l}0.58 \\
\pm 0.45\end{array}$ & $\begin{array}{l}1.57 \\
\pm 1.05\end{array}$ \\
\hline $\begin{array}{l}\text { myocyte } \\
\text { diameter }\end{array}$ & $\begin{array}{l}14.1 \\
\pm 03.2\end{array}$ & $\begin{array}{l}18.1 \\
\pm 4.1\end{array}$ & $\begin{array}{l}11.3 \\
\pm 2.6\end{array}$ & $\begin{array}{l}16.7 \\
\pm 4.5\end{array}$ & $\begin{array}{l}14.5 \\
\pm 3.3\end{array}$ & $\begin{array}{r}18.4 \\
\pm 4.2\end{array}$ & $\begin{array}{l}11.5 \\
\pm 2.7\end{array}$ & $\begin{array}{l}17.1 \\
\pm 4.4\end{array}$ & $\begin{array}{l}13.1 \\
\pm 2.7\end{array}$ & $\begin{array}{l}17.2 \\
\pm 3.7\end{array}$ & $\begin{array}{r}10.9 \\
\pm 2.4\end{array}$ & $\begin{array}{l}15.5 \\
\pm 4.6\end{array}$ \\
\hline ST vs. LT & & $\mathrm{p}<\mathrm{N} . \mathrm{S}$ & & $<<0.001$ & & & & & & & & \\
\hline
\end{tabular}

Figure 6. Anatomical distribution of fibrosis and myocyte hypertrophy. A) Examples of the LT RA, showing larger intermyocyte distances in the thin epicardial layer compared to the underlying trabeculae (left photograph) and on the epicardial side of a thicker part of the wall compared to the endocardial side (right photograph). The bar chart compares intermyocyte distances in Thin: the outer millimeter of thin parts of the atrial walls (local wall thickness $\leq 1 \mathrm{~mm}$ ), Thick: the outer millimeter of thick parts of the atrial walls (local wall thickness $>1 \mathrm{~mm}$ ) and Trab: the endocardial trabeculae. B) Overview of distances (mean \pm s.d.) between myocyte bundles, between myocytes within bundles and myocyte diameter in total, in the outer millimeter of the atrial walls and in deeper layers (distance $>1 \mathrm{~mm}$ from the epicardium).

\subsection{High-resolution optical mapping of wavefront propagation}

To delineate the consequences of structural remodeling for epicardial wavefront propagation, we performed high-resolution optical mapping during local stimulation (BCL $350 \mathrm{~ms}$ at $1.1 \mathrm{x}$ threshold) in $2 \times 2 \mathrm{~cm}$ areas of ST and LT left atrial free walls. Afterwards, the bundle orientation within the recorded areas was reconstructed and superimposed on activation patterns (figure 7A). 
The direction of fastest propagation agreed well with the epicardial fiber orientation at the stimulus site (figure 7B). Figure 7C shows examples of waves in 2 ST and 2 LT LA. In the ST LA, epicardial wavefront spread rapidly with some degree of anisotropy. In the LT LA, wavefronts expanded more slowly and had a more irregular shape. Figure 7D shows the characteristics of epicardial wavefront expansion. The size of waves increased significantly more slowly in LT LA than ST LA (left panel). As the wavefronts spread out and encountered different fiber orientations, the degree of anisotropy decreased in both ST and LT LA (middle panel). However, the degree of anisotropy was higher in LT LA than in ST LA in the first milliseconds of wavefront propagation. In ST LA, the wavefront was also more circular than in LT LA (right panel), reflecting the more irregular shape of wavefronts in LT goats.

\subsection{Effect of endomysial fibrosis in a dual-layer atrial model}

To investigate whether increased endomysial fibrosis in the epicardial layer can explain the more complex fibrillation patterns and in particular the increased incidence of epicardial breakthroughs, we developed a simplified 'proof-of-principle' computer model consisting of an interconnected epicardial and endocardial layer (figure $8 \mathrm{~A}$ ). In this model, endomysial fibrosis (simulated by removing 2 out of 3 transverse connections at random in the epicardial layer) significantly increased the number of simultaneous fibrillation waves and the incidence of epicardial breakthroughs, paralleling the observed differences between ST and LT goats in epicardial mapping experiments. 

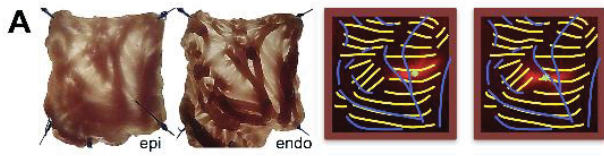

B
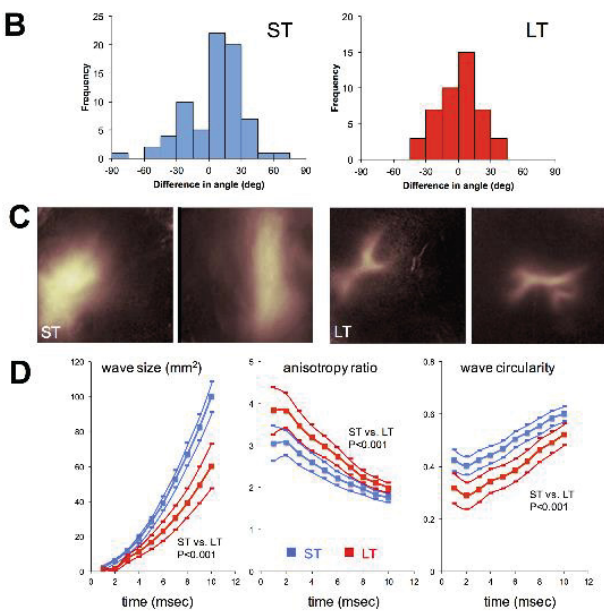

Figure 7. Difference in wavefront expansion between the ST and LT LA. A) Left panels: LA recording location from an LT goat, excised and back-lit to visualize the epicardial fiber orientation (epi) and the endocardial trabeculae (endo, mirrored). Right panels: reconstructed bundle orientations (yellow for epi, blue for endo) from the recording location superimposed on frames taken $5 \mathrm{~ms}$ after stimulation at the sites indicated by asterisks. B) For each stimulation site, the difference in angle between the reconstructed bundle orientation and the orientation of fastest wavefront spread (obtained by fitting the wavefront with an ellipse) was determined. Histograms consist of data for all stimulation sites in all goats. For both the ST and LT group, there was a good correlation between the reconstructed bundle orientation and the direction of fastest wavefront spread. C) Examples of frames taken $5 \mathrm{~ms}$ after stimulation in 2 ST goats (left panels) and 2 LT goats (right panels). In LT goats, wavefronts had a more irregular shape. D) Wavefront characteristics as a function of time after stimulation: total wave size (left panel), the anisotropy ratio (middle panel, calculated as the major axis/ minor axis of an ellipse fitted to the shape of the wavefront) and wave circularity $\left(4 \pi *\left(\right.\right.$ area/perimeter $\left.{ }^{2}\right)$, right panel). Bars represent $95 \%$ confidence intervals. In the LT LA, wavefront spread was slower, more anisotropic in the first milliseconds and more irregularly shaped. 

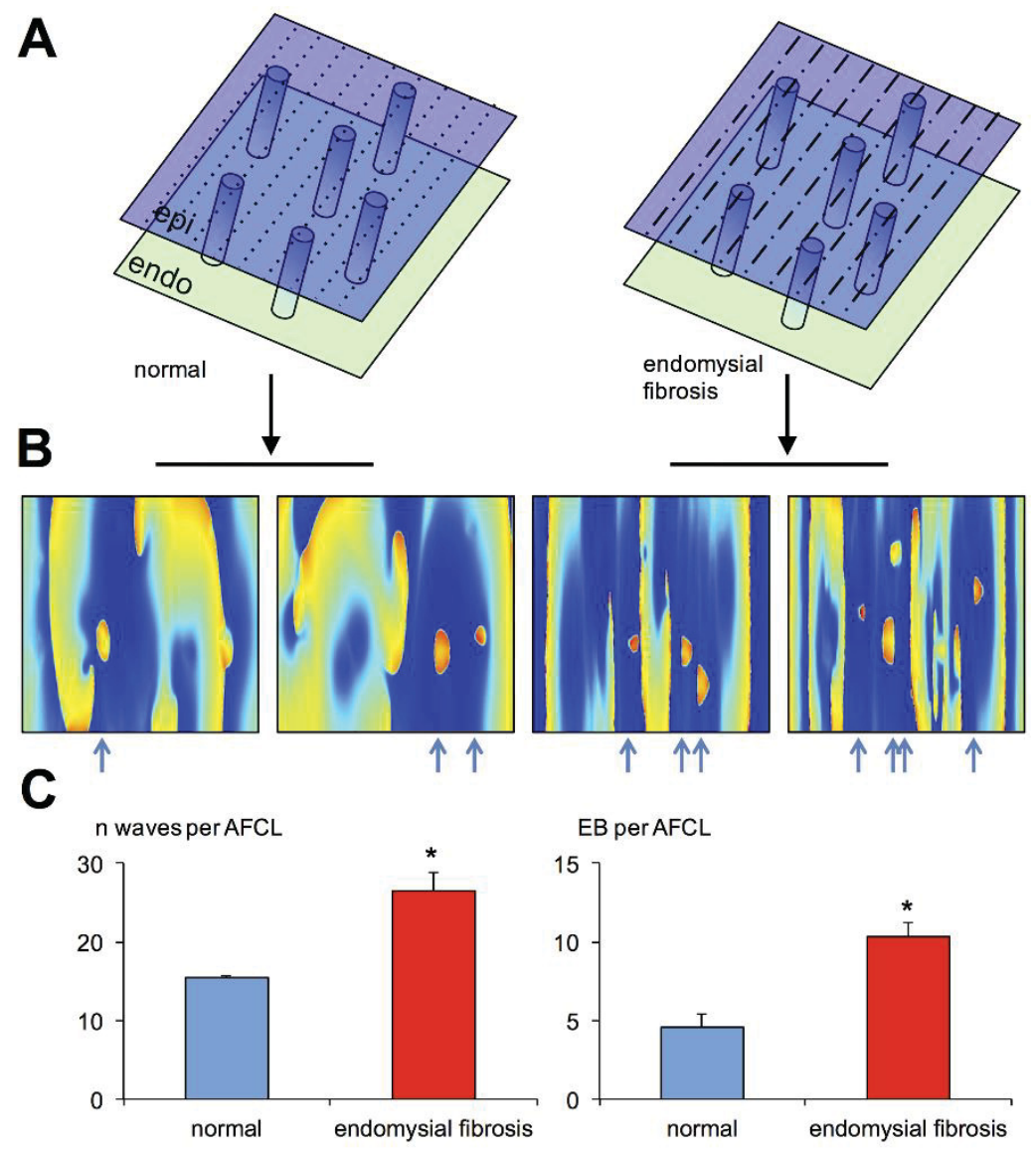

Figure 8. Effect of epicardial endomysial fibrosis in a mathematical model. A) Schematic representation of the proof-of-principle model. To simulate epicardial endomysial fibrosis, 2 out of 3 transverse electrical connections were removed at random. B) Frames showing epicardial activations patterns without (left panels) and with (right panels) endomysial fibrosis. Arrows indicate positions of epicardial breakthroughs. C) Simulated endomysial fibrosis caused a significant increase in the number of waves and the incidence of epicardial breakthroughs (mean \pm s.e.m., $* p<0.01$ ). 


\section{Discussion}

In the goat model used in this study, AF leads to atrial electrical and structural remodeling, both of which are thought to make AF more stable. ${ }^{5}$ Electrical remodeling (a shortening of the action potential duration and effective refractory period) takes places within the first 1-3 days of AF. In this early phase, induced AF episode durations increase from seconds to minutes. ${ }^{2}$ In the ensuing months, AF stability further increases, in parallel with the slow process of structural remodeling. ${ }^{12}$ AF can still be cardioverted pharmacologically after 3 weeks (persistent AF), but not after 6 months (permanent AF). ${ }^{4}$ Here, we have used these time-points to investigate how the transition from persistent to permanent $A F$ is related to structural remodeling.

We have previously reported that AF in LT goats was characterized by a higher number of simultaneous waves (both peripheral waves and EBs) and a larger incidence of conduction block, leading to a more dissociated and complex fibrillation pattern compared to AF in ST goats. Our analysis of fibrillation patterns in this study shows that LT goats had less clustering and thus more homogeneity in the spatial distribution of the entry points of peripheral waves and EBs and of the occurrence of slow conduction. The combination of a higher degree of complexity and lower degree of clustering indicates an underlying process causing changes in conduction that is more widespread and homogeneously distributed in LT goats than in ST goats. The lower degree of clustering of entry points into the recorded area (both of peripheral waves and EBs) also suggests that the possible contribution of localized high-frequency sources outside the recorded area is smaller in LT than in ST goats. These data are in agreement with several studies in patients showing that, compared to acute and paroxysmal AF, conduction patterns in longstanding persistent $\mathrm{AF}$ are more complex and conduction defects more widespread. ${ }^{6-9}$ The observations that paroxysmal AF is often driven by sources in the pulmonary vein region, but that in longstanding persistent AF the entire atria contribute to the AF substrate, may explain the lower efficacy of ablation strategies in longstanding persistent AF patients. ${ }^{11,18}$ 
To characterize the pattern of structural remodeling within the complex anatomy of the atria, we quantified the transmural distribution of fibrous tissue and myocyte diameters. We did not observe systematic differences between the appendages, trabeculated anterior regions and non-trabeculated posterior regions of the atrial free walls (data not shown). Myocyte hypertrophy was observed throughout the atrial wall and may have contributed to altered conduction. Based on detailed models of atrial tissue structure, Spach et al. have proposed that myocyte hypertrophy can lead to more pronounced propagation delays during transverse propagation. ${ }^{14}$

The difference in the transmural distributions of endomysial fibrosis and myocyte hypertrophy suggests that these two processes are caused by different stimuli (e.g. stretch vs. neurohumoral activation). For example, it is conceivable that the thin epicardial layer is subjected to a larger degree of stretch than the underlying network of trabeculae, providing a larger stimulus for reactive fibrosis in the epicardial layer.

The endomysial pattern of fibrosis we have observed, is thought to arise in reaction to certain stimuli, e.g. stretch and pressure overload in the absence of myocyte death, as opposed to replacement fibrosis, which occurs as a repair mechanism after myocyte death. ${ }^{19}$ Indeed, in the goat AF model, myocytes undergo dedifferentiation without an increase in apoptosis. ${ }^{20}$ There are some interesting differences with the nature and distribution of structural remodeling in another well-established animal model of AF, the canine model of congestive heart failure (CHF) due to rapid ventricular pacing. In CHF dogs compared to control dogs, a large increase was observed in the area fraction of fibrous tissue in the atria, as quantified from Sirius Red stainings. ${ }^{21}$ By contrast, the LT group in our study did not have a larger area fraction of fibrous tissue than the ST goats, as quantified in Toluidine blue stainings. Although different stainings were used in these studies, the methods for quantification are similar. In addition, other studies on animal models of AF due to rapid atrial pacing have also shown little or no increase in the area fraction of fibrous tissue. ${ }^{21,22}$ Histological analysis in canine CHF model showed large areas of fibrosis after 
5 weeks of ventricular pacing, following an early phase of necrosis and apoptosis (within 24 hours after the start of pacing). ${ }^{23}$ Thus, the pattern of fibrosis in CHF dogs is highly suggestive of replacement fibrosis after earlier damage to the atrial myocardium, as opposed to the reactive fibrosis pattern in LT goats. Interestingly, the pattern of fibrillatory conduction in CHF model is relatively simple, and consistent with macro-reentry, ${ }^{24,25}$ as opposed to the complex fibrillation patterns observed in LT goats. ${ }^{4}$ Although a direct comparison is not straightforward due to the different species and other confounding factors, this supports the notion that the exact type and distribution of fibrous tissue is at least as important as the overall degree of fibrosis. ${ }^{15}$

An increase in interstitial fibrosis is also seen during normal aging, ${ }^{26-}$ ${ }^{28}$ which may in part explain why age is a major independent risk factor for AF. In a sense, AF may cause 'accelerated aging' of the atrial myocardium. Spach and coworkers have demonstrated that this type of 'microfibrosis' leads to discontinuous transverse propagation on a microscopic scale due to a loss of side-to-side connections between myocytes (reviewed in ${ }^{13}$ ). The resulting 'nonuniform anisotropy' allows for reentry to occur in small circuits. ${ }^{13,27}$ These studies were performed in isolated bundles or atrial regions with a highly organized fiber orientation, whereas the atrial free walls display more variability. This is reflected in the pattern of wavefront spread from a point source in our study, which showed high anisotropy close to the site of stimulation that decreased as the wavefront spread out and encountered a variety of fiber orientations. The differences between ST and LT goats in wavefront expansion are consistent with a loss of side-to-side connections due to endomysial fibrosis in LT goats. In optical mapping recordings, we have only investigated propagation during slow pacing to assess the effect of structural remodeling with fully recovered sodium current availability. Conduction heterogeneity is likely to be more pronounced at shorter intervals, with incomplete recovery of the sodium current (e.g. during extrastimulation or AF). ${ }^{28,} 29$

In LT goats, endomysial fibrosis occurred predominantly in the thin epicardial layer and may therefore have specific effects on 
propagation within the complex atrial anatomy. Scheussler et al. showed in isolated canine atria that the epicardium and endocardium can show regional differences in activation time and that reentry pathways may involve the transmural plane. ${ }^{30}$ In a study on isolated sheep right atria, Berenfeld et al have shown how the endocardial network of trabeculae can increase the complexity of fibrillation patterns. ${ }^{31}$ Houben et al have proposed that with a loss of the 'leading role' of the thin epicardial layer, the underlying trabecular network may become dominant, resulting in a more disorganized and stable type of $\mathrm{AF}^{32}$ This is in agreement with the higher incidence of epicardial breakthroughs due to transmural conduction that has been observed in patients with permanent (or longstanding persistent) $\mathrm{AF}^{9}$ Using simultaneous epi- and endocardial mapping, we have recently shown that the degree of epi-endocardial dissociation of fibrillation waves is substantially larger in LT goats than in ST goats. ${ }^{17}$ The endomysial fibrosis observed in the present study is likely to lead to a loss of continuity of the thin epicardial layer. This would reduce its synchronizing effect on fibrillatory propagation in the underlying trabecular network, increasing the tortuosity of reentrant pathways and creating a more 3-dimensional substrate for AF. This hypothesis was strengthened by simulations using a simplified model of two interconnected layers, where transverse uncoupling in the epicardial layer caused an increase in 3-dimensional conduction, as evidenced by the increased incidence of epicardial breakthroughs.

\section{Limitations and Clinical Relevance}

The goat model represents AF without preexisting structural heart disease. By contrast, a majority of patients are of advanced age or have some form of structural heart disease that leads to a substrate for AF (often including atrial fibrosis) before the first onset of AF. However, the goat model allows study of the consequences of AF itself. It is conceivable that $A F$, even with a preexisting substrate, causes specific structural changes (e.g. endomysial fibrosis) that gradually increase AF stability. Prevention of these changes may form an important target for treatments 
strategies to maintain sinus rhythm. The circumstances leading to endomysial fibrosis differ from those leading to replacement fibrosis. Therefore, the underlying signaling pathways are likely to differ, which may allow specific upstream therapy.

The dual-layer mathematical model does not represent the full structural complexity of the atrial anatomy, and conduction patterns in a more complex model are likely to be quantitatively different. However, it was intended as a proof-of-principle model to test the hypothesis that transverse uncoupling in the epicardium will lead to a more 3 -dimensional conduction pattern, as reflected by an increased incidence of epicardial breakthroughs.

We propose that structural changes in LT goats, most notably endomysial fibrosis in the epicardial layer, are responsible for the increased complexity of fibrillatory conduction. We cannot directly prove a causal relation, in part because of the difference in scale between changes in structure (micrometers) and behavior of fibrillation waves (millimeters to centimeters). Nevertheless, we propose that we present strong evidence for this relation because: 1. Both the alterations in structure and conduction are ubiquitously and homogeneously present in the epicardial layer. 2. Our results are in agreement with relation between endomysial fibrosis and impaired transverse propagation in detailed studies on isolated atrial muscle bundles. ${ }^{13} 3$. The results of high-resolution optical mapping recordings are consistent with transverse uncoupling of myocyte fibers. 4. Changes in epicardial conduction were reproduced in a simple model of two interconnected layers. Taken together, this evidence suggests that specific inhibition of endomysial fibrosis may represent a promising target to prevent the progressive stabilization of AF. 


\section{References:}

1. Kopecky S, Gersh B, McGoon M, Whisnant J, Holmes D, Ilstrup $D$, Frye R. The natural history of lone atrial fibrillation. A population-based study over three decades. N Engl J Med. 1987;317:669-674

2. Wijffels M, Kirchhof C, Dorland R, Allessie M. Atrial fibrillation begets atrial fibrillation. A study in awake chronically instrumented goats. Circulation. 1995;92:1954-1968

3. Eijsbouts S, Ausma J, Blaauw Y, Schotten U, Duytschaever M, Allessie M. Serial cardioversion by class ic drugs during 4 months of persistent atrial fibrillation in the goat. Journal of cardiovascular electrophysiology. 2006;17:648-654

4. Verheule S, Tuyls E, van Hunnik A, Kuiper M, Schotten U, Allessie M. Fibrillatory conduction in the atrial free walls of goats in persistent and permanent atrial fibrillation. Circulation Arrhythmia and electrophysiology. 2010;3:590-599

5. Schotten U, Verheule S, Kirchhof P, Goette A. Pathophysiological mechanisms of atrial fibrillation: A translational appraisal. Physiological reviews. 2011;91:265-325

6. Ravelli F, Faes L, Sandrini L, Gaita F, Antolini R, Scaglione M, Nollo G. Wave similarity mapping shows the spatiotemporal distribution of fibrillatory wave complexity in the human right atrium during paroxysmal and chronic atrial fibrillation. Journal of cardiovascular electrophysiology. 2005;16:1071-1076

7. Wu J, Estner H, Luik A, Ucer E, Reents T, Pflaumer A, Zrenner B, Hessling G, Deisenhofer I. Automatic 3d mapping of complex fractionated atrial electrograms (cfae) in patients with paroxysmal and persistent atrial fibrillation. Journal of cardiovascular electrophysiology. 2008;19:897-903

8. Allessie MA, de Groot NMS, Houben RPM, Schotten U, Boersma E, Smeets JL, Crijns HJ. Electropathological substrate of longstanding persistent atrial fibrillation in patients with structural heart disease: Longitudinal dissociation. Circulation Arrhythmia and electrophysiology. 2010;3:606-615

9. de Groot NMS, Houben RPM, Smeets JL, Boersma E, Schotten U, Schalij MJ, Crijns H, Allessie MA. Electropathological substrate of longstanding persistent atrial fibrillation in patients with structural heart disease: Epicardial breakthrough. Circulation. 2010;122:1674-1682 
10. Haissaguerre M, Jais $P$, Shah D, Takahashi A, Hocini M, Quiniou G, Garrigue S, Le Mouroux A, Le Metayer P, Clementy J. Spontaneous initiation of atrial fibrillation by ectopic beats originating in the pulmonary veins. N Engl J Med. 1998;339:659666

11. Sanders $P$, Berenfeld $O$, Hocini M, Jaïs $P$, Vaidyanathan R, Hsu L-F, Garrigue S, Takahashi Y, Rotter M, Sacher F, Scavée C, Ploutz-Snyder R, Jalife J, Haïssaguerre M. Spectral analysis identifies sites of high-frequency activity maintaining atrial fibrillation in humans. Circulation. 2005;112:789-797

12. Ausma J, Litjens N, Lenders M, Duimel H, Mast F, Wouters L, Ramaekers F, Allessie M, Borgers M. Time course of atrial fibrillation-induced cellular structural remodeling in atria of the goat. Journal of molecular and cellular cardiology. 2001;33:20832094

13. Spach M, Boineau J. Microfibrosis produces electrical load variations due to loss of side-to-side cell connections: A major mechanism of structural heart disease arrhythmias. Pacing Clin Electrophysiol. 1997;20:397-413

14. Spach M, Heidlage J, Barr R, Dolber P. Cell size and communication: Role in structural and electrical development and remodeling of the heart. Heart Rhythm. 2004;1:500-515

15. Burstein B, Nattel S. Atrial fibrosis: Mechanisms and clinical relevance in atrial fibrillation. Journal of the American College of Cardiology. 2008;51:802-809

16. Ho S, Anderson R, Sanchez-Quintana D. Atrial structure and fibres: Morphologic bases of atrial conduction. Cardiovascular research. 2002;54:325-336

17. Eckstein J, Maesen B, Linz DK, Zeemering S, van Hunnik A, Verheule S, Allessie MA, Schotten U. Time course and mechanisms of endo-epicardial electrical dissociation during atrial fibrillation in the goat. Cardiovascular research. 2011;89:816-824

18. Sanders P, Nalliah CJ, Dubois R, Takahashi Y, Hocini M, Rotter M, Rostock T, Sacher F, Hsu L-F, Jönsson A, O\&apos;Neill MD, Jaïs $P$, Haïssaguerre $M$. Frequency mapping of the pulmonary veins in paroxysmal versus permanent atrial fibrillation. Journal of cardiovascular electrophysiology. 2006;17:965-972

19. Weber K, Pick R, Jalil J, Janicki J, Carroll E. Patterns of myocardial fibrosis. Journal of molecular and cellular cardiology. 1989;21 Suppl 5:121-131 
20. Dispersyn G, Ausma J, Thone F, Flameng W, Vanoverschelde J, Allessie M, Ramaekers F, Borgers M. Cardiomyocyte remodelling during myocardial hibernation and atrial fibrillation: Prelude to apoptosis. Cardiovascular research. 1999;43:947-957

21. Li D, Fareh S, Leung T, Nattel S. Promotion of atrial fibrillation by heart failure in dogs: Atrial remodeling of a different sort. Circulation. 1999;100:87-95

22. Ausma J, van der Velden H, Lenders M, van Ankeren E, Jongsma $H$, Ramaekers F, Borgers M, Allessie M. Reverse structural and gap-junctional remodeling after prolonged atrial fibrillation in the goat. Circulation. 2003;107:2051-2058

23. Cardin S, Li D, Thorin-Trescases N, Leung T, Thorin E, Nattel S. Evolution of the atrial fibrillation substrate in experimental congestive heart failure: Angiotensin-dependent and independent pathways. Cardiovascular research. 2003;60:315325

24. Li D, Benardeau A, Nattel S. Contrasting efficacy of dofetilide in differing experimental models of atrial fibrillation. Circulation. 2000;102:104-112

25. Ryu K, Sahadevan J, Khrestian CM, Stambler BS, Waldo AL. Use of fast fourier transform analysis of atrial electrograms for rapid characterization of atrial activation-implications for delineating possible mechanisms of atrial tachyarrhythmias. Journal of cardiovascular electrophysiology. 2006;17:198-206

26. Spach M, Dolber P. Relating extracellular potentials and their derivatives to anisotropic propagation at a microscopic level in human cardiac muscle. Evidence for electrical uncoupling of sideto-side fiber connections with increasing age. Circulation research. 1986;58:356-371

27. Koura T, Hara M, Takeuchi S, Ota K, Okada Y, Miyoshi S, Watanabe A, Shiraiwa K, Mitamura H, Kodama I, Ogawa S. Anisotropic conduction properties in canine atria analyzed by high-resolution optical mapping: Preferential direction of conduction block changes from longitudinal to transverse with increasing age. Circulation. 2002;105:2092-2098

28. Spach M, Heidlage J, Dolber P, Barr R. Mechanism of origin of conduction disturbances in aging human atrial bundles: Experimental and model study. Heart Rhythm. 2007;4:175-185

29. Verheule S, Wilson EE, Banthia S, Everett TH, Shanbhag S, Sih $\mathrm{HJ}$, Olgin JE. Direction-dependent conduction abnormalities in a canine model of atrial fibrillation due to chronic atrial dilatation. 
American journal of physiology Heart and circulatory physiology. 2004;287:H634-644

30. Schuessler R, Kawamoto T, Hand D, Mitsuno M, Bromberg B, Cox J, Boineau J. Simultaneous epicardial and endocardial activation sequence mapping in the isolated canine right atrium. Circulation. 1993;88:250-263

31. Berenfeld O, Zaitsev A, Mironov S, Pertsov A, Jalife J. Frequency-dependent breakdown of wave propagation into fibrillatory conduction across the pectinate muscle network in the isolated sheep right atrium. Circulation research. 2002;90:11731180

32. Houben RPM, de Groot NMS, Smeets JLRM, Becker AE, Lindemans FW, Allessie MA. S-wave predominance of epicardial electrograms during atrial fibrillation in humans: Indirect evidence for a role of the thin subepicardial layer. Heart Rhythm. 2004;1:639-647 
Chapter 4 


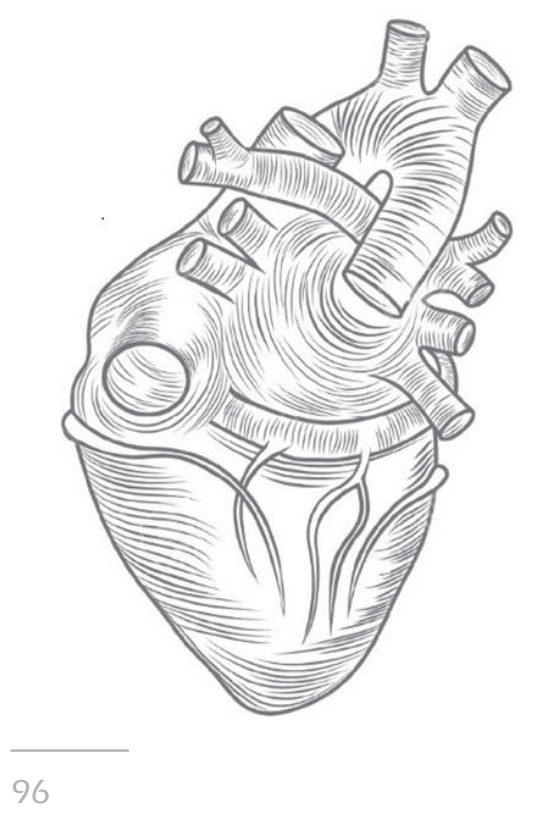




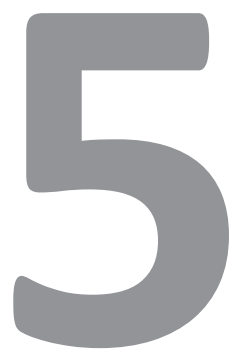

Effect of $\mathrm{Na}^{+}$channel blockade on the threedimensional substrate of atrial fibrillation in a model of endo-epicardial dissociation and transmural conduction

Ali Gharaviri

Sander Verheule

Jens Eckstein

Mark Potse

Nico H.L. Kuijpers

Ulrich Schotten

Dept. of Physiology, Maastricht University, Maastricht, The Netherlands.

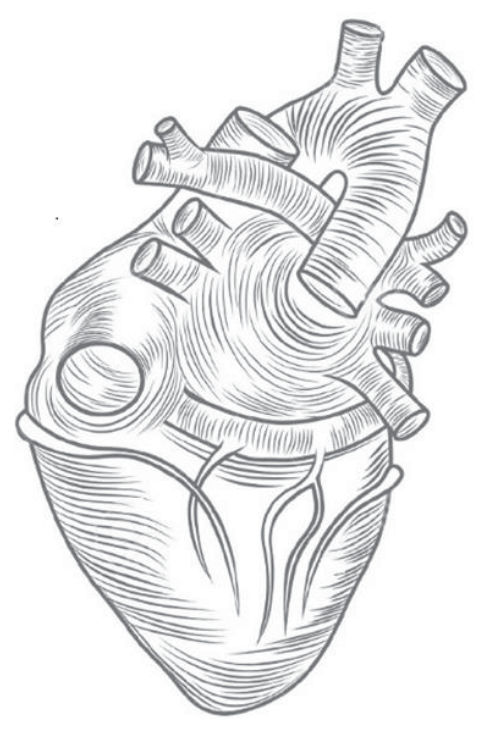




\section{Abstract}

\section{Introduction:}

Atrial fibrillation (AF) is a progressive arrhythmia characterized by structural alterations that increase its stability. Both clinical and experimental studies show a concomitant loss of antiarrhythmic drugs efficacy in both experimental models and patients in later stage of AF. We have simulated the efficacy of sodium channel blocker in the presence of different degrees of structural remodeling during AF.

\section{Methods}

In a computer model consisting of 2 cross-connected atrial layers, with realistic atrial membrane behavior, endo-epicardial conduction was simulated. Progressive structural remodeling was simulated by reducing the connectivity between the two layers. $100 \%$ endoepicardial connectivity represented a healthy atrium. At various degrees of structural remodeling, we assessed the effect of $60 \%$ sodium channel block on AF stability, endo-epicardial electrical activity dissociation (EED), and complexity of fibrillatory conduction such as number of waves, phase singularities (PSs) and transmural conduction ('breakthrough', BT) in comparison with no sodium channel block (control).

\section{Results:}

$\mathrm{Na}$-block terminated $\mathrm{AF}$ in non-remodeled but not in remodeled atria. The temporal excitable gap and atrial fibrillation cycle length increased at all degrees of remodeling compared to control. Despite the increase of EED and excitable gap, Na-block decreased the incidence of BT because of transmural conduction block. The number of waves and phase singularities (PSs) decreased in nonremodeled atrium in the presence of sodium channel block compared to the control but remained unaltered in the structurally remodeled atrium. 


\section{Conclusions:}

This simplified atrial model mimics the loss of efficacy of $\mathrm{Na-block}$ in terminating AF in the presence of severe structural remodeling as has been observed experimentally and clinically. AF termination in atria with moderate structural remodeling in presence of $\mathrm{Na}$ block is caused by reduction of AF complexity. With more severe structural remodeling, $\mathrm{Na}$-block fails to promote synchronization of the two atrial layers of the model. 


\section{Introduction}

Atrial fibrillation (AF) is the most common tachyarrhythmia. ${ }^{1} \mathrm{AF}$ progression is caused by electrical and structural remodeling. Electrical remodeling entails shortening of the action potential and effective refractory period (ERP), which develops within the first days of AF. Structural remodeling develops more slowly over a time course of months to years and is considered to be the main mechanism leading to AF progression. ${ }^{2}$ Several studies have demonstrated that structural remodeling is associated with a decrease in the size of fibrillatory waves, an increased dissociation in electrical activity between epicardial layer and endocardial bundle network, and an increase in the incidence of transmural conduction ("breakthroughs"). ${ }^{3-5}$

Several therapeutic strategies are used in AF treatment, ranging from pharmaceutical interventions to ablation. Anti-arrhythmic drugs have been an important part of AF therapy for many years. ${ }^{6}$ Among all anti-arrhythmic drugs, sodium channel blockers have a long history in AF treatment. Recent studies have increased their interest in $\mathrm{Na}$-channel blockers such as flecainide for AF therapy. ${ }^{7}$ Although several studies have been performed on the effects of the sodium channel blockers on atrial fibrillatory conduction, the actual mechanisms of electrical cardioversion are not well understood. So far both experimental and modeling studies proposed three possible mechanisms by which sodium channel blockers possibly could terminate AF. These mechanisms include: (1) enlargement of PS tips (2) decreased anchoring of rotors to functional obstacles, and (3) reduction in number of waves, due to an increase in the excitable gap (EG). ${ }^{8}$

Despite the fact that all simulation studies reported a high success rate in AF termination by the application of sodium channel blockers, several experimental studies have revealed a reduction in termination rates after administration of sodium channel blockers in both animal models and patients in later stages of AF. Unfortunately the mechanisms underlying this loss of efficacy were not fully understood. In a recent study performed by Eckstein et al, the three-dimensional substrate of atrial fibrillation, which could 
have an important effect on sodium channel block efficacy on AF termination, was investigated. In this study we demonstrated that flecainide reduced the incidence of BTs and endo-epicardial electrical dissociation significantly. ${ }^{9}$ Since all simulation studies on the effect of sodium channel blocker on AF termination were performed in a two dimensional sheets of atrial myocytes, in this study we used a previously developed dual layer model ${ }^{10-12, \text { chapter } 3}$ which enabled us to study the effect of three-dimensional substrate on $\mathrm{Na}$-channel blocker efficacy. In the present study we investigated why a $\mathrm{Na-channel} \mathrm{blocker} \mathrm{is} \mathrm{effective} \mathrm{in} \mathrm{early} \mathrm{stages}$ of $A F$ but gradually loses its efficacy in the later stages of $A F$ or, more precisely, with progressive structural remodeling. We hypothesized that the possible explanation for this loss is that structurally remodeled atria represent a higher degree of 3dimensionality in the AF substrate. We addressed this hypothesis by assessing the effect of $\mathrm{Na}$-channel blockade on endo-epicardial electrical activity dissociation, transmural conduction and $\mathrm{AF}$ stability at different degrees of structural remodeling.

\section{Methods}

\subsection{Model}

To investigate the effect of sodium channel blockade on AF stability, endo-epicardial dyssynchrony and breakthrough events during atrial fibrillation, we used a dual-layer computer model (figure $1 \mathrm{~A})^{10, \text { chapter } 2}$

Human atrial electrophysiology was modeled with a mono-domain reaction-diffusion model comprising two layers with a size of $4 \mathrm{~cm}$ $\times 4 \mathrm{~cm}$. Each of the layers was composed of $400 \times 400$ segments with a size of $0.01 \mathrm{~cm} \times 0.01 \mathrm{~cm}$. Ionic currents and calcium handling for each segment was described by the Courtemanche-RamirezNattel model. ${ }^{13}$ Conductivities were the same in both directions, $\sigma_{\mathrm{x}}=\sigma_{\mathrm{y}}=0.5 \mathrm{mS} / \mathrm{cm}$, which implies isotropic tissue. Total ionic membrane current was given by: 
$I_{\text {ion }}=I_{\mathrm{Na}}+I_{\mathrm{K} 1}+I_{\mathrm{to}}+\mathrm{I}_{\mathrm{Kur}}+\mathrm{I}_{\mathrm{Kr}}+\mathrm{I}_{\mathrm{Ks}}+\mathrm{I}_{\mathrm{Ca}, \mathrm{L}}+\mathrm{I}_{\mathrm{p}, \mathrm{Ca}}+\mathrm{I}_{\mathrm{NaK}}+\mathrm{I}_{\mathrm{NaCa}}+\mathrm{I}_{\mathrm{b}, \mathrm{Na}}+$

$\mathrm{I}_{\mathrm{b}, \mathrm{Ca}}$

where $\mathrm{I}_{\mathrm{Na}}$ is fast inward $\mathrm{Na}^{+}$current, $\mathrm{I}_{\mathrm{K} 1}$ is inward rectifier $\mathrm{K}^{+}$current, $\mathrm{I}_{\text {to }}$ transient outward $\mathrm{K}^{+}$current, $\mathrm{I}_{\mathrm{kur}}$ is ultrarapid delayed rectifier $\mathrm{K}^{+}$ current, $\mathrm{I}_{\mathrm{kr}}$ is rapid delayed rectifier current, $\mathrm{I}_{\mathrm{Ks}}$ is slow delayed rectifier $\mathrm{K}^{+}$current, $\mathrm{I}_{\mathrm{Ca}, \mathrm{L}}$ is $\mathrm{L}$-type $\mathrm{Ca}^{2+}$ current, $\mathrm{I}_{\mathrm{p}, \mathrm{Ca}}$ is $\mathrm{Ca}^{2+}$ pump current, $I_{N a K}$ is $\mathrm{Na}^{+}-\mathrm{K}^{+}$pump current, $I_{\mathrm{NaCa}}$ is $\mathrm{Na}^{+} / \mathrm{Ca}^{2+}$ exchanger current, and $\mathrm{I}_{\mathrm{b}, \mathrm{Na}}$ and $\mathrm{I}_{\mathrm{b}, \mathrm{Ca}}$ are background $\mathrm{Na}^{+}$and $\mathrm{Ca}^{2+}$ currents. To incorporate changes in ionic currents that have been observed in atrial fibrillation, conductivities for $\mathrm{I}_{\mathrm{to}}, \mathrm{I}_{\mathrm{Ca}, \mathrm{L}}$, and $\mathrm{I}_{\mathrm{K} 1}$ were set at $40 \%$, $35 \%$, and $200 \%$ of the default values, respectively. ${ }^{2}$

Electrical coupling between the two layers was implemented by adding an Ohmic conductor between opposing segments at socalled connection points. Each connection point was a circle with a radius of $0.1 \mathrm{~cm}$.

\subsection{Simulation protocol}

To investigate the effect of ion channel blockers on endo-epicardial electrical activity dissociation and transmural conduction at different stages of structural remodeling, the following simulation protocol was applied (see figure 1C):

1. In one layer, a spiral wave was initiated using an S1-S2 protocol, while the other layer was quiescent, as described in previous study. ${ }^{10}$ The simulation was continued for 1 second in order to achieve a stable spiral wave.

2. One second after the start of the simulation, 6 connection points were added at randomly chosen sites, with the constraint that two connection points were at least 0.15 $\mathrm{cm}$ apart. To exclude possible bias resulting from a particular geometry of connection points, 8 different geometries with 6 randomly chosen sites were created. For each of the 8 geometries, the simulation of step 1 was continued for 6 more seconds. This resulted in 8 different simulations that were used as a starting point of the next stages of the simulation. 
3. The 8 simulations from step 2 were used and continued for another 6 seconds, either without changing the connection points or after adding more randomly chosen connection points (at least $0.15 \mathrm{~cm}$ apart), so that the total number of connections was $6,12,24,48$ or 96 . Simultaneously. In addition, each simulation from step 2 was continued as well with $100 \%$ connectivity, which means that all opposing segments in the two layers were connected to one another.

4. All simulations at step 3 were simulated twice, once with the normal sodium conductance (control) and once with $60 \%$ sodium channel conductance block. As described above, steps 2 and 3 were performed 8 times such that in total 48 simulation runs were performed in step 3 .

In our model, 6 and 12 connections represented severely remodeled atria; 24 and 48 connections represented moderately remodeled atria, and 96 connections and $100 \%$ connectivity represented a healthy atrium.

\subsection{Analysis}

In all 48 simulations of step 3 the following parameters were analyzed (for algorithms used for these analyses see appendix A):

- Number of phase singularities (PSs)

- Number of fibrillation waves

- Atrial fibrillation cycle length (AFCL)

- Temporal excitable gap (EG)

- Degree of endo-epicardial dyssynchrony

- Breakthrough rates (BTRs)

- Atrial fibrillation stability 
A

B
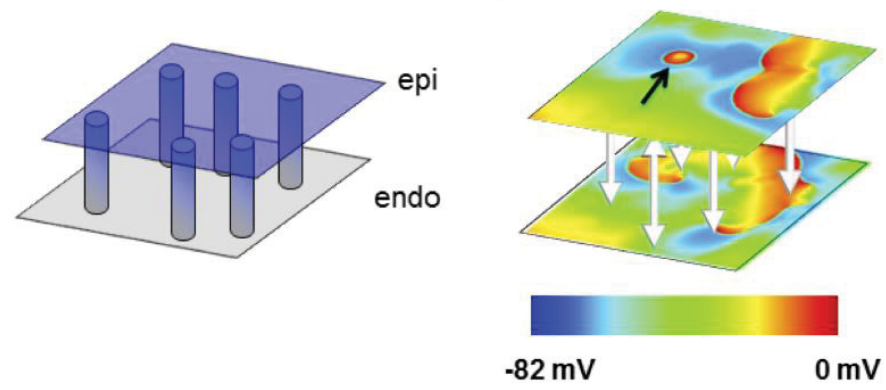

C

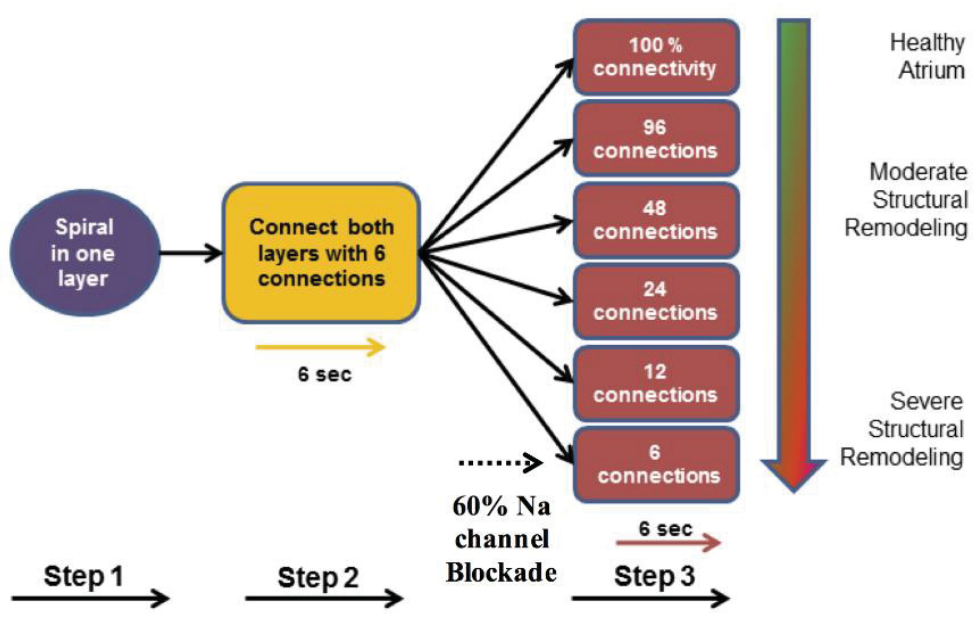

Figure1. Dual layer model of the atrial wall (A) Model structure with two layers (epi- and endocardium) and transmural connections (grey cylinders). (B) Dissociation of electrical activity between the two layers and transmural conduction resulting in a breakthrough wave (black arrow). (C) Simulations protocol (see methods section for details). 


\section{Results}

Figure 2 shows representative examples of AF in control and in the presence of $\mathrm{Na}$-block for two different degrees of endo-epicardial connectivity (96 and 12 connections).

With 96 connections - representing a healthy atrium - a large number of BTs occurred immediately after the additional connections were introduced, leading to effective synchronization of the layers. Complete synchronization of the two layers in average took approximately $250 \mathrm{~ms}$. After synchronization, no BTs were observed (see Figure 2B), and the AF episode quickly terminated.

In contrast to healthy atrium with 12 connections, BTs occurred throughout the simulation, but electrical activity did not synchronize between the layers.

\subsection{AF stability}

We assessed the effect of sodium channel blockade on AF stability. The Kaplan-Meier curves in figure $3 A$ showed the AF persistence in different degrees of connectivity. In simulations with high number of connections, 96 connections and $100 \%$ connectivity, Na-channel blockade led to faster AF termination compared to control. With 48 and 24 connections, AF persistence was comparable between control and $\mathrm{Na}$-channel blockade. With a low number of connections, 6 and 12 connections, stability of AF was similar or slightly more stable with $\mathrm{Na}$-channel blockade, compared to control. 


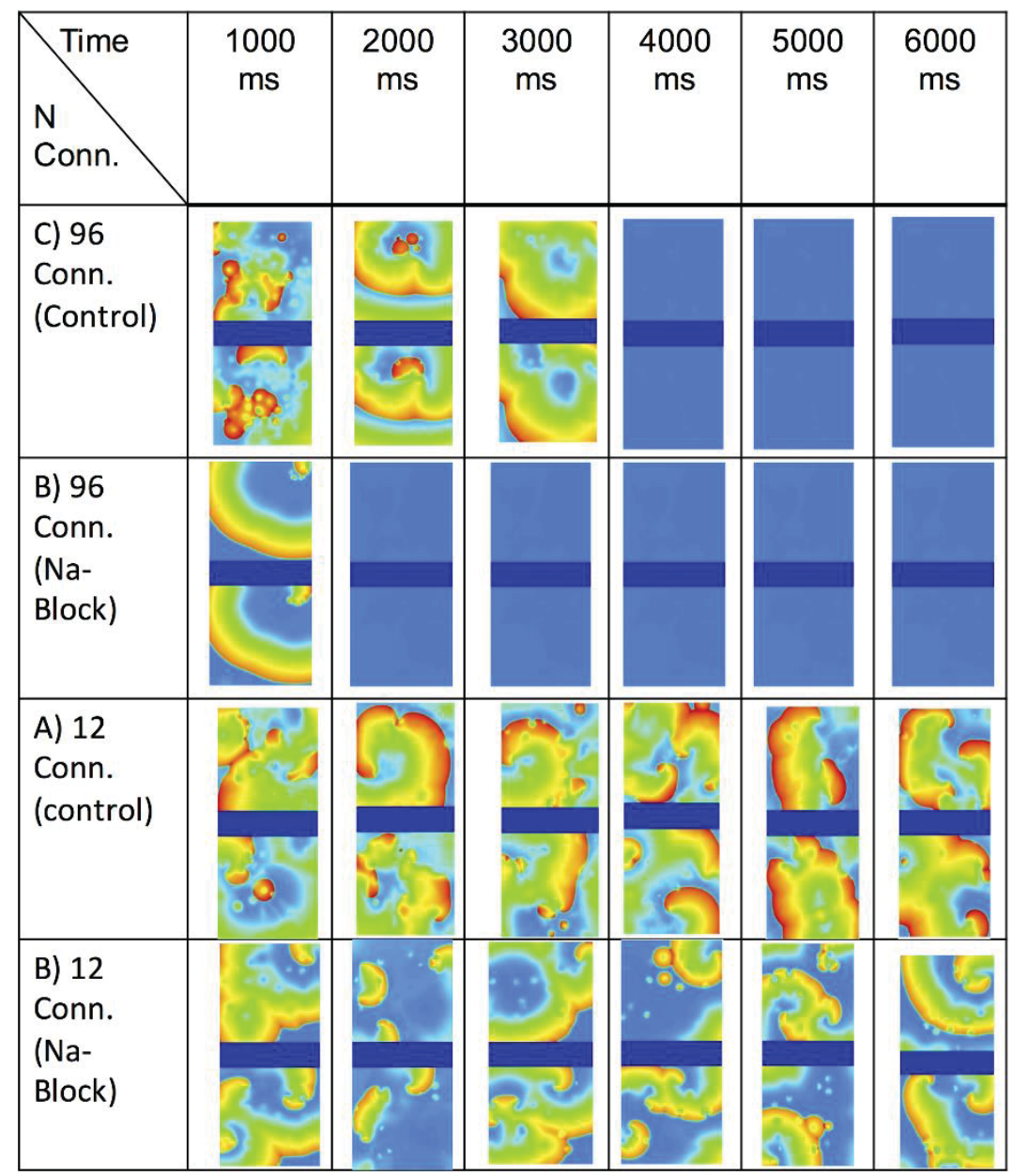

Figure 2. Colour-coded membrane potentials of representative simulations with two different degrees of endo-epicardial electrical coupling. A and C) Simulations without $\mathrm{Na}$-channel blocker. B and D) Simulations with $\mathrm{Na}$-channel blocker. The epicardial layer is shown in the top, the endocardial in the bottom panel. Snapshots are shown for every second of a simulation. 


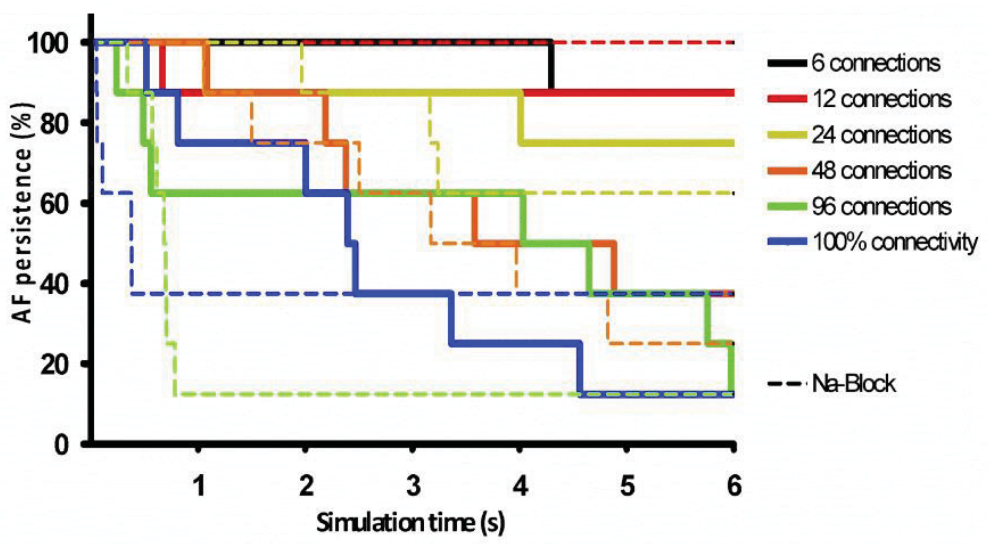

Figure3. AF persistence. Kaplan-Meier curves showing atrial fibrillation persistence with and without $\mathrm{Na}$ channel blockade (solid line = control, dashed line $=\mathrm{Na}$ channel blockade) in different sets of simulations with $6,12,24,48,96$ connections, and $100 \%$ connectivity ( $n=8$ for all different sets of simulations).

\subsection{Effect of sodium channel blockade on fibrillation patterns}

As described above, Na-channel blockade only decreased AF persistence with 96 connections and $100 \%$ connectivity. To further assess the mechanisms of AF termination, we computed several electrophysiological parameters in both groups (figure 4). As expected, $\mathrm{Na}$-channel blockade increased the atrial fibrillation cycle length (AFCL) and the excitable gap (EG) (figure $4 A$ and $B$ ). The number of waves in both groups was similar, except at high degrees of connectivity, where $\mathrm{Na}$-channel block decreased the number of waves (figure 4C). Similarly, $\mathrm{Na}$-channel block decreased the number of PSs only for 96 connections and $100 \%$ connectivity (figure 4D). Dyssynchrony was not affected by $\mathrm{Na}$-channel blockade, and the BT rate showed a non-significant trend towards a decrease in the presence of $\mathrm{Na}-$ channel block (figure $4 \mathrm{E}$ and $\mathrm{F}$ ). 
AFCL (ms)

$\mathbf{A}$

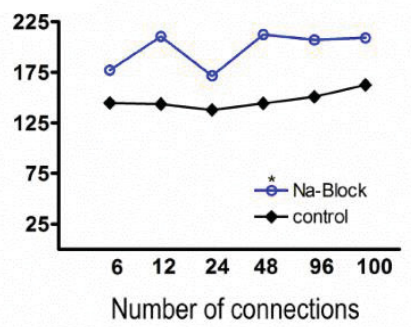

C

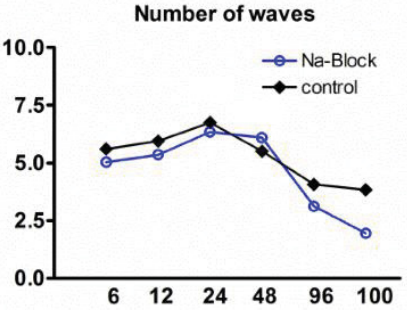

$\mathbf{E}$

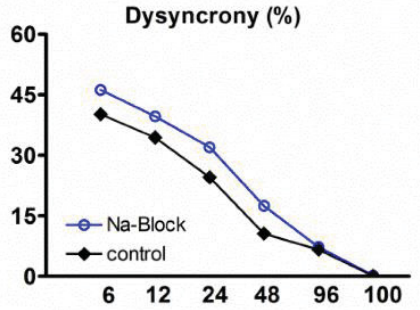

EG (ms)

B

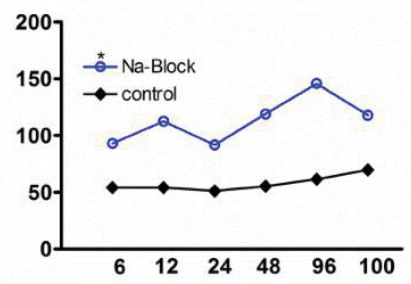

D

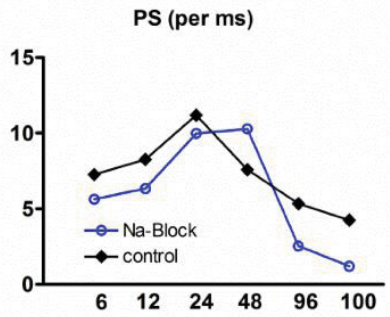

F

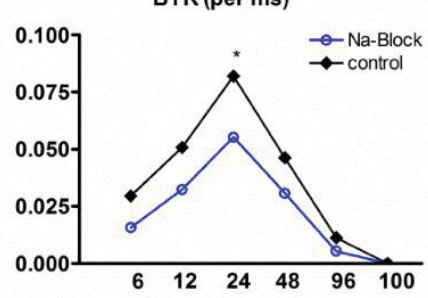

Figure 4. Electrophysiological parameters calculated in simulations with (blue) and without $\mathrm{Na}$ channel blockade (black). A) AF cycle length. B) Excitable gap. C) Number of waves. D) Number of phase singularities. E) Electrical activity dyssynchrony between two layers. F) Breakthrough rate per ms.

In order to analysis further the loss of AF termination by sodium channel blocker, we compared the simulations with severe (6 and 12 connections) and no (96 connections and 100\% connectivity) structural remodeling and grouped them in two groups called 3D and $2 \mathrm{D}$ respectively. In figure 5 , a pronounced loss of efficacy of sodium channel blockade is observed in the $3 \mathrm{D}$ group. 
In both $2 \mathrm{D}$ and $3 \mathrm{D}$ groups the presence of sodium channel block also significantly increased both the AFCL and EG compared to the control (see figure $5 \mathrm{~A}$ and $5 \mathrm{~B}$ ).

AF pattern complexity, quantified as the number of waves and PSs, in the 2D groups was significantly lower in the presence of sodium channel blocker compared to control. Interestingly, the sodium channel blocker did not have this effect in the 3D simulation group (see figure $5 \mathrm{C}$ and $\mathrm{D}$ ). BTs are a reflection of 3-dimensional substrate of $A F$. As illustrated in the figure $5 E$, sodium channel blockade reduced significantly the number of BTs in the 3D group. A pronounced increase in the number of unsuccessful BTs as a result of $\mathrm{Na}$-channel blockade was observed in all set of connections (figure 6).

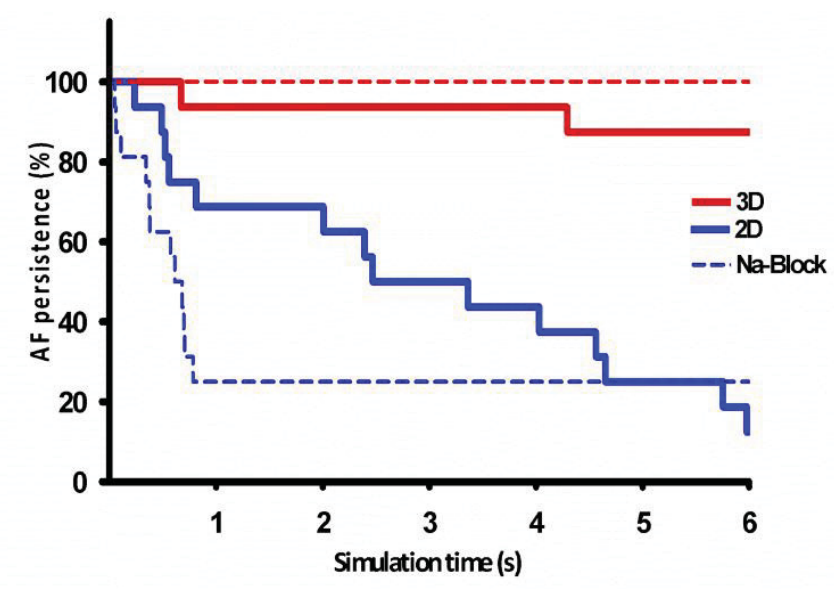

Figure5. AF persistence. Kaplan-Meier curve showing atrial fibrillation persistence with and without $\mathrm{Na}$ channel blockade (solid line = control, dashed line $=\mathrm{Na}$ channel blockade) in simulations entitled in 2D and $3 D$ group ( $n=16$ for all different sets of simulations). 

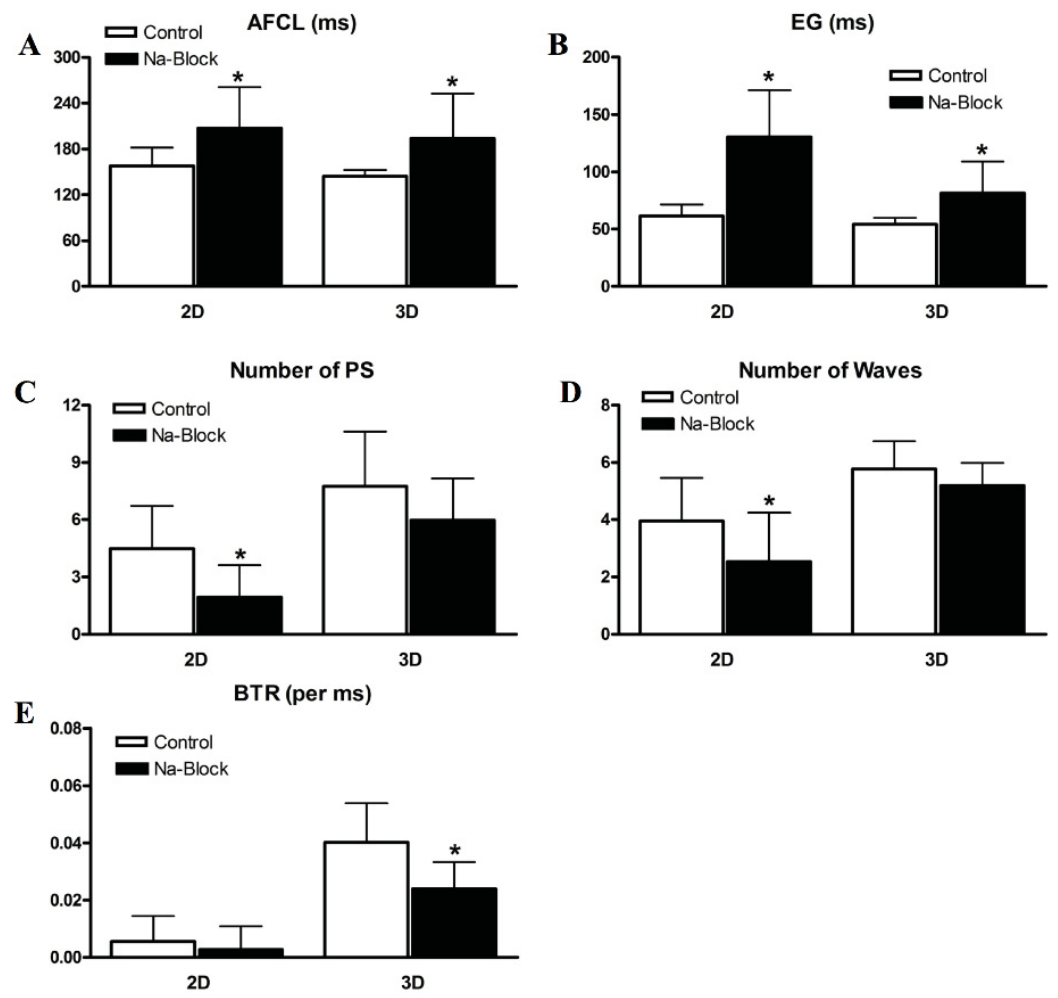

Figure 6. Measured electrophysiological parameters in slight structural remodeled (2D) and severe structural remodeled (3D) atrial model in control and in the presence of $\mathrm{Na}$-channel block ( $\mathrm{Na}-\mathrm{Block}$ ). A) AFCL.

B) EG. C) Number of PSs. D) Number of waves. E) Breakthrough rate.

\section{Discussion}

Despite the fact that the effects of sodium channel blockade on AF have been extensively studied, mechanism of AF termination by $\mathrm{Na}$-block and the reason for the loss of efficacy in structurally remodeled atria are poorly understood. Interestingly, some effects of flecainide may even be pro-arrhythmic such as a decrease in conduction velocity. The decreases in conduction velocity will as a 
consequence shorten the "wavelength" (refractory period $\times$ conduction velocity). ${ }^{14}$

In contrast, functional conduction block at pivot points due to temporal excitable gap prolongation has been proposed as the main mechanism leads to AF termination. ${ }^{8,14-16} 17$ Hence, PSs are more likely to meander and collide with the atrial border, leading to termination of that PS.

Although simulation studies investigated several mechanisms underlying $\mathrm{AF}$ termination in the presence of $\mathrm{Na}$-channel block, the question regarding the loss of efficacy of $\mathrm{Na}$-channel block in later stages of $A F$ is still unanswered. In a goat model of $A F$, we demonstrated that this loss of efficacy was not due to a loss of $\mathrm{AFCl}$ prolongation in the presence of the drug. ${ }^{18}$ Rather, loss of $\mathrm{AF}$ termination could be due to the increasingly 3 dimensional conduction pattern of $A F$ in later stages of remodeling. We recently investigated the effect of flecainide on the 3-dimensional characteristics of $\mathrm{AF}^{.}{ }^{9}$ We demonstrated a decrease in transmural conductions and endo-epicardial electrical activity dissociation in goat $\mathrm{AF}$ model in presence of flecainide. We also observed reduction of flecainide to terminate $\mathrm{AF}$ at later stage of $\mathrm{AF}$ in the goat model.

\subsection{Effect of $\mathrm{Na}$-blocker on AF stability}

In the present study we showed the efficacy loss of $\mathrm{Na}$-channel blocker in AF termination in different stages of structural remodeling. In all simulations with no structural remodeling (96 and $100 \%$ connectivity) AF episodes terminated much faster in the presence of the $\mathrm{Na}$-channel blocker. However in all simulations with severe structural remodeling ( 6 or 12 connections), $\mathrm{Na}-$ channel blockade was unable to terminate AF. Interestingly, we did not observe significant differences in $\mathrm{AF}$ termination rate in simulations with moderate structural remodeling (24 and 48 connections. A possible reason could be the more 3-dimensional character of conduction in strongly structural remodeled substrates compared to the more 2D character of conduction patterns in simulations with well-coupled layers. 


\subsection{Parameters determining AF stability}

As illustrated in the figure 4 and 6, AFCL and EG were increased in the presence of $\mathrm{Na}$-channel blockade. Hence this increase was present in all different number of connections and also in both 2D and $3 \mathrm{D}$ groups compared to the control, it cannot be an explanation for the loss of efficacy with structural remodeling in our model. These findings were in agreement with findings reported in previous studies. $^{8,17}$

The presence of $\mathrm{Na}$-channel blocker decreased significantly the AF complexity, quantified as the number of waves and phase singularities, only in the 2D group but not in the 3D group compared to the control. This inability of $\mathrm{Na}$-channel blocker to reduce AF complexity in severe structural remodeling, which has a more 3dimensional character, could be an answer for the $\mathrm{Na}$-channel blocker efficacy loss at the later stage of AF.

\subsection{The effect of $\mathrm{Na}$-channel blocker on dyssynchrony and BTs}

In the goat atria, we recently showed a decrease in both endoepicardial dissociation and BTs in the presence of flecainide. ${ }^{9}$ In our model, we observed only a slight, but insignificant increase in dyssynchrony as a result of $\mathrm{Na}$ channel blockade, regardless of the degree of connectivity. At first glance, a similar degree of dyssynchrony in combination with an increase in EG should increase the number of opportunities for BTs to occur. However, we observed a decrease in BT incidence (see figure 3F). To investigate possible mechanisms for this reduction, we analyzed number of unsuccessful BTs (figure 7B). Unsuccessful BT was defined as a BT that did not conduct transmurally despite the presence of local endo-epicardial electrical dyssynchrony. A pronounced increase in the number of unsuccessful BTs as a result of $\mathrm{Na}$-channel blockade was observed in all set of connections (figure 7C). This transmural conduction block due to reduced excitability or a sink/source mismatch could be the possible explanation for surprisingly low number of breakthroughs in the presence of $\mathrm{Na}$ channel block (given the increase in excitable gap). 
However, the decline of the breakthrough rate in the presence of $\mathrm{Na}$ channel block was obviously not strong enough to allow for sufficient reduction of AF complexity and AF termination.
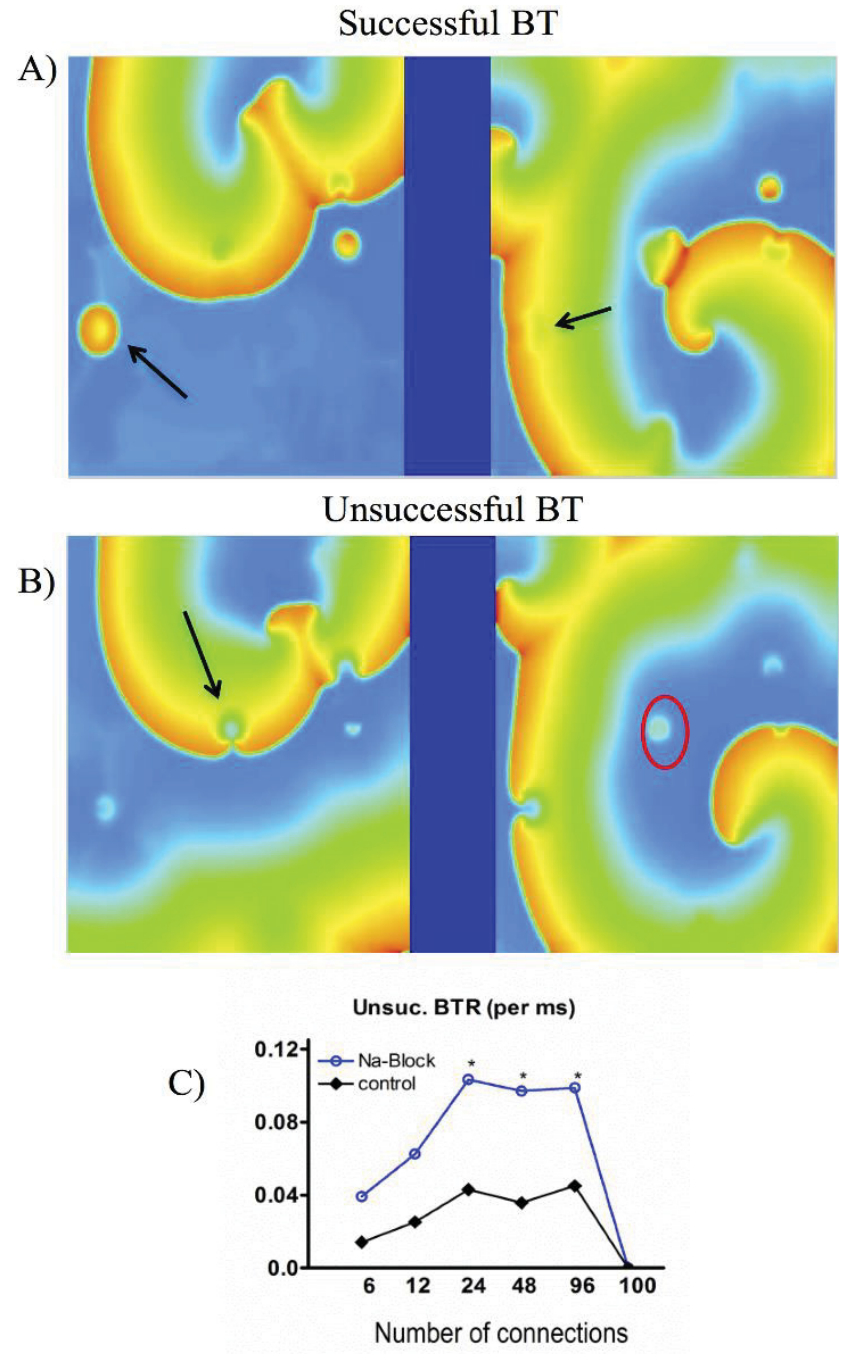

Figure 7. Examples of $A$ ) a successful $B T$ and $B$ ) an unsuccessful BT (red circle). C) The number of unsuccessful BTs in both control and $\mathrm{Na}$-blockade group. 


\section{Study Limitations}

Our simplified dual-layer model should be considered as a proofof-principle model. This model does not reflect 3D geometry and complex structure of the atrium, fiber orientation arrangement, heterogeneity in ionic membrane currents, and variability in atrial wall thickness. According to this, the effect of myocardial thickness could not be addressed. In this study a simple poreblock model (a fixed percentage decrease in maximum Na_conductance) was used to simulate $\mathrm{Na}_{-}$-channel blockade. A more detailed exploration of the impact of state-dependent $I_{N a}$ block as occurs for all clinically used class I drugs, with the application of a mathematical formulation of such action, would be of great interest but is beyond the scope of the present study. However, the work in this study does demonstrate for the first time that pure reduction of $\mathrm{Na}^{+}$current fails to terminate $\mathrm{AF}$ in the presence of severe structural remodeling leading to a threedimensional substrate for AF.

We also did not consider ectopic focal discharges as a mechanism contributing to AF maintenance in this study. Despite these limitations, we have shown that our model is well suited to investigate the effect of $\mathrm{Na}$-channel blockers on endo-epicardial dissociation, breakthroughs and AF stability. 


\section{References:}

1. Nattel S, Shiroshita-Takeshita A, Brundel BJ, Rivard L. Mechanisms of atrial fibrillation: lessons from animal models. Progress in cardiovascular diseases Jul-Aug 2005;48:9-28.

2. Schotten U, Verheule S, Kirchhof P, Goette A. Pathophysiological mechanisms of atrial fibrillation: a translational appraisal. Physiological reviews Jan 2011;91:265-325.

3. Verheule S, Tuyls E, van Hunnik A, Kuiper M, Schotten U, Allessie M. Fibrillatory conduction in the atrial free walls of goats in persistent and permanent atrial fibrillation. Circulation Arrhythmia and electrophysiology Dec 2010;3:590-599.

4. de Groot NM, Houben RP, Smeets JL, Boersma E, Schotten U, Schalij MJ, Crijns H, Allessie MA. Electropathological substrate of longstanding persistent atrial fibrillation in patients with structural heart disease: epicardial breakthrough. Circulation Oct 26 2010;122:1674-1682.

5. Allessie MA, de Groot NM, Houben RP, Schotten U, Boersma E, Smeets JL, Crijns HJ. Electropathological substrate of longstanding persistent atrial fibrillation in patients with structural heart disease: longitudinal dissociation. Circulation Arrhythmia and electrophysiology Dec 2010;3:606-615.

6. Comtois $P$, Sakabe $M$, Vigmond EJ, Munoz M, Texier A, Shiroshita-Takeshita A, Nattel S. Mechanisms of atrial fibrillation termination by rapidly unbinding $\mathrm{Na}+$ channel blockers: insights from mathematical models and experimental correlates. American journal of physiology Heart and circulatory physiology Oct 2008;295:H1489-1504.

7. Nattel S, Carlsson L. Innovative approaches to anti-arrhythmic drug therapy. Nature reviews Drug discovery Dec 2006;5:10341049 .

8. Kneller J, Kalifa J, Zou R, Zaitsev AV, Warren M, Berenfeld O, Vigmond EJ, Leon LJ, Nattel S, Jalife J. Mechanisms of atrial fibrillation termination by pure sodium channel blockade in an ionically-realistic mathematical model. Circulation research Mar 18 2005;96:e35-47.

9. Eckstein J. The three-dimensional substrate of atrial fibrillation in the goat. Maastricht2012.

10. Gharaviri A, Verheule S, Eckstein J, Potse M, Kuijpers NH, Schotten U. A computer model of endo-epicardial electrical 
dissociation and transmural conduction during atrial fibrillation. Europace : European pacing, arrhythmias, and cardiac electrophysiology : journal of the working groups on cardiac pacing, arrhythmias, and cardiac cellular electrophysiology of the European Society of Cardiology Nov 2012;14 Suppl 5:v10-v16.

11. Verheule S, Tuyls E, Gharaviri A, Hulsmans S, van Hunnik A, Kuiper M, Serroyen J, Zeemering S, Kuijpers NH, Schotten U. Loss of continuity in the thin epicardial layer because of endomysial fibrosis increases the complexity of atrial fibrillatory conduction. Circulation Arrhythmia and electrophysiology Feb 2013;6:202-211.

12. Verheule S, Eckstein J, Linz D, Maesen B, Bidar E, Gharaviri A, Schotten U. Role of endo-epicardial dissociation of electrical activity and transmural conduction in the development of persistent atrial fibrillation. Progress in biophysics and molecular biology Aug 2014;115:173-185.

13. Courtemanche M, Ramirez RJ, Nattel S. Ionic mechanisms underlying human atrial action potential properties: insights from a mathematical model. The American journal of physiology Jul 1998;275:H301-321.

14. Danse PW, Garratt CJ, Allessie MA. Flecainide widens the excitable gap at pivot points of premature turning wavefronts in rabbit ventricular myocardium. Journal of cardiovascular electrophysiology Sep 2001;12:1010-1017.

15. Eckstein J, Verheule S, de Groot NM, Allessie M, Schotten U. Mechanisms of perpetuation of atrial fibrillation in chronically dilated atria. Progress in biophysics and molecular biology JunJul 2008;97:435-451.

16. Danse PW, Garratt CJ, Mast F, Allessie MA. Preferential depression of conduction around a pivot point in rabbit ventricular myocardium by potassium and flecainide. Journal of cardiovascular electrophysiology Mar 2000;11:262-273.

17. Wijffels MC, Kirchhof CJ, Dorland R, Allessie MA. Atrial fibrillation begets atrial fibrillation. A study in awake chronically instrumented goats. Circulation Oct 1 1995;92:1954-1968.

18. Eijsbouts S, Ausma J, Blaauw Y, Schotten U, Duytschaever M, Allessie MA. Serial cardioversion by class IC Drugs during 4 months of persistent atrial fibrillation in the goat. Journal of cardiovascular electrophysiology Jun 2006;17:648-654. 
Chapter 5 


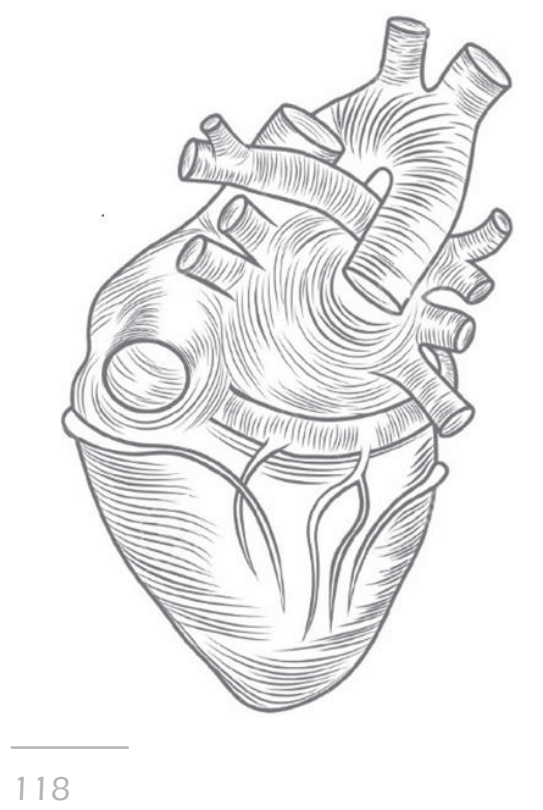




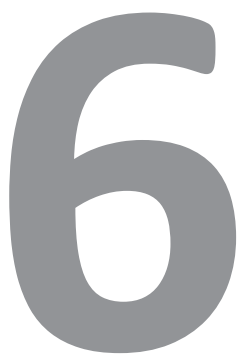

A 3D model of endo-epicardial dissociation and transmural conduction during atrial fibrillation

Ali Gharaviri

Mark Potse

Sander Verheule

Ulrich Schotten

Dept. of Physiology, Maastricht University, Maastricht, The Netherlands. In preparation of submission

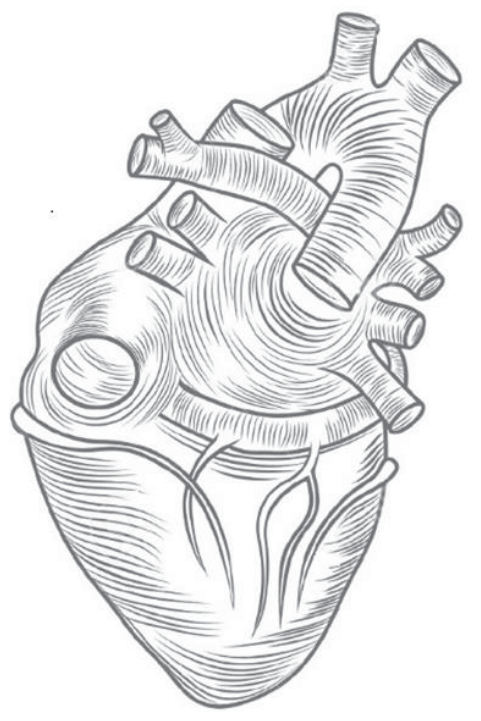




\section{Abstract}

\section{Introduction:}

Loss of side-to-side electrical connections between atrial muscle bundles is thought to contribute to conduction disturbances that lead to atrial fibrillation (AF). Experimental studies suggest that increased epicardial fibrosis leads to dissociation of electrical activity between epicardial and endocardial bundle network, thus enhancing transmural conduction ("breakthrough").

\section{Methods}

We built a novel anatomical model of the atria implementing an epicardial layer with realistic fiber orientations and endocardial muscle bundles. We tested the hypothesis that epicardial fibrosis enhances endo-epicardial dissociation (EED) and breakthrough (BT). Fibrosis was modeled by randomly removing transverse electrical connections in the epicardial layer.

\section{Results:}

With no, slight, moderate, and severe fibrosis, AF complexity, quantified by the number of fibrillation waves increased from 3.8 to $5.95,8.8$ and 18.2 and number of phase singularities increased from 4.6 to $10.2,16.5$, and 20.3 respectively. Along with increasing AF complexity, EED increased from 10.8 to $13.8,16.8$, and $18.8 \%$. In the presence of fibrosis endo-epicardial conduction occurred with breakthroughs showing radial spread of activation in the epicardial layer. The number of BTs in 3 seconds of simulated AF increased from 64 without fibrosis to 138 with severe fibrosis.

\section{Conclusions:}

This model is the first anatomical atrial model of AF to display BTs, a common and conspicuous feature of fibrillation patterns in experimental studies. An increase in fibrosis in the epicardial layer increased the degree of EED and the incidence of BTs, thus 
increasing the complexity of fibrillatory conduction. The model offers the opportunity to study transmural conduction and its contribution to AF complexity in more detail. 


\section{Introduction}

The perpetuation and progression of atrial fibrillation (AF) is mediated by electrical and structural remodeling the atria. ${ }^{1}$ Electrical remodeling is a relatively fast process (1-2 days) that includes action potential shortening and reduction of effective refractory period. Structural remodeling develops much more slowly (months-years) and is considered as the main mechanism leading to AF stability. Several studies have demonstrated that structural remodeling is associated with a decrease in the size of fibrillatory waves and an increased incidence of transmural conduction ("breakthroughs") in both patients and goat models. ${ }^{2-4}$ Verheule et al. demonstrated that transition from persistent to permanent AF in goats is histologically characterized by an increase in endomysial fibrosis, particularly in the outer millimeter of the atrial wall. ${ }^{5}$ This increase leads to loss of continuity in the epicardial layer due to loss of side-to-side connections. ${ }^{5,6}$ In this way, endo-epicardial transmural connectivity and electrical synchronization between endo- and epicardium would be hampered. Using simultaneous endo-epicardial mapping, Eckstein et al. showed a significant increase in endo-epicardial electrical dissociation (EED) as well as breakthroughs (transmurally propagating fibrillation waves) in goats in later stages of $\mathrm{AF}^{7,8}$

While these experimental studies show a clear association between structural remodeling, EED, and breakthrough incidence, they cannot establish a causal relationship or elucidate which aspects of the structural remodeling are responsible for the observed changes. The purpose of this study was to assess the effects of loss of side-to-side connectivity in the epicardial layer on EED and breakthrough incidence, using a numerical model in which all other factors were unchanged. We created a new anatomical model of the atria, which improved in several ways on previous models to make simulations of transmural conduction in the atria possible. The main feature implemented in this model is the combination of an epicardial layer with anisotropic tissue characteristics with an endocardial muscle bundles network showing varying degrees of coupling with the thin epicardial layer. Simulated AF with an 
increasing degree of epicardial transverse uncoupling resulted in increased EED and breakthroughs, demonstrating that reduced side-to-side connectivity is a key player in transmural conduction during AF. To our knowledge this is the first model allowing evaluation of this mechanism in an anatomical in-silico model of AF. Additionally, using this model we could investigate the translation of AF conduction pattern complexity on body surface and transesophageal electrograms.

\section{Methods}

\subsection{D Model Structure}

An anatomical 3D model of human atria including fiber orientation was built manually using Blender (The Blender Foundation, Amsterdam, The Netherlands) as a 3D editing tool. The global shape was extracted from magnetic resonance imaging data of a subject with normal atrial anatomy. Endocardial and epicardial surfaces were created and combined into a 'myocardial envelope': a closed surface containing the atrial myocardium. 20 Pectinate muscles $(P M)$ in the right atrial (RA) free wall, Bachmann's Bundle (BB), inter atrial bundles, and the crista terminalis (CT) were added based on published anatomical descriptions. ${ }^{9-11}$ The model contained four pulmonary veins, caval veins, left and right atrial appendages (LAA and RAA), as well as the coronary sinus, as illustrated in figure1A, $B$ and $C$. The $B B$ connected a region next to the superior caval vein to the anterosuperior wall of the left atrium. The coronary sinus (CS) originated in the RA and extended along the left atrial (LA) posterior wall. A limited number of fiber tracks connected the CS to the posterior inferior LA.

Dedicated software was used to create a computational mesh at $0.2-\mathrm{mm}$ resolution with different fiber orientations from the manually-edited objects. Within the myocardial envelope a 1-mm layer of myocardium was placed to represent the thin right-atrial wall. A 3-mm layer was used for the thicker left atrium. The bundles consisted of myocardium to which different conduction properties could be assigned. In this software bundles were drawn to define 
fiber orientations in different regions of the atrium. These bundles are called ghost bundle and did not contribute tissue.

\subsection{Fiber orientation and anisotropy}

One of the most important properties of this model is the incorporation of realistic fiber orientation based on histological observations (see figure $1 \mathrm{C}$ and D). ${ }^{9}$ Ho et al have described the left-atrial wall as 2 layers with different fiber orientations. ${ }^{9}$ Less information is available for the right atrium. A study by Maesen et al. revealed that the right atrial fiber orientation in the epicardial surface is perpendicular to the endocardial bundle network in goats. $^{12}$

In our model, elements within the bundles received a fiber orientation parallel to the local bundle orientation. For the left-atrial wall, two sets of ghost bundles were used to confer different fiber orientations on the endocardial and epicardial half of the wall. Each model element took its orientation from the nearest bundle. In the right-atrial wall the orientation was governed by the nearest true bundle, and was made perpendicular to it, but parallel to the epicardial surface. An additional set of ghost bundles was used to confer a circumferential fiber orientation on the elements in the pulmonary and caval vein ostia.

\subsection{Electrophysiological model}

Simulations were performed with the propag-5 software ${ }^{13,14}$ and run on 2560 cores of a Cray XE6 supercomputer. Electrical activity was simulated with a mono-domain reaction-diffusion equation. Ionic currents and calcium handling for each model node was described by Courtemanche-Ramirez-Nattel model. ${ }^{15}$

To reproduce electrical remodeling as observed in atrial fibrillation, conductivities for $I_{\mathrm{to}}, I_{\mathrm{caL}}$, and $I_{\mathrm{k} 1}$ were set at $15 \%, 15 \%$, and $200 \%$ of their normal values respectively. ${ }^{1}$ 
A)
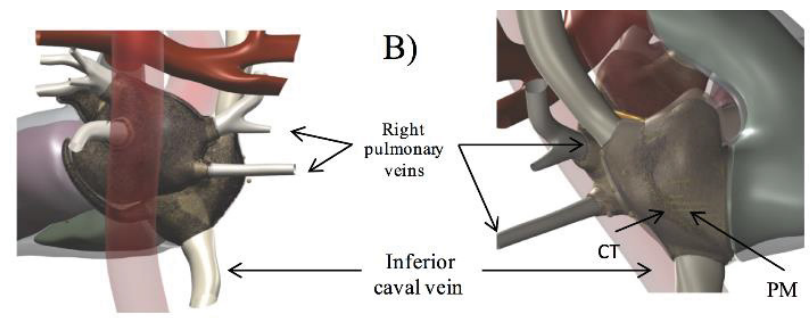

C)

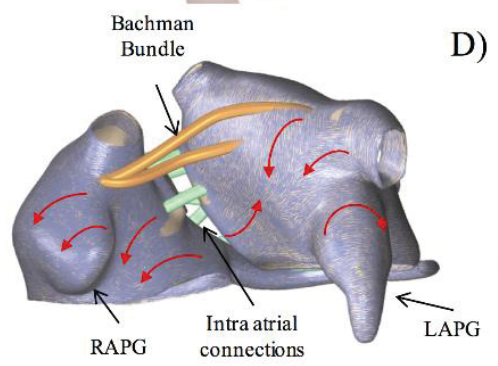

D)

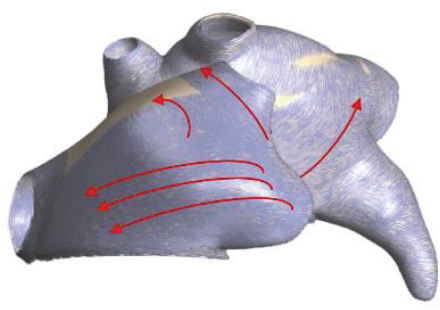

E)

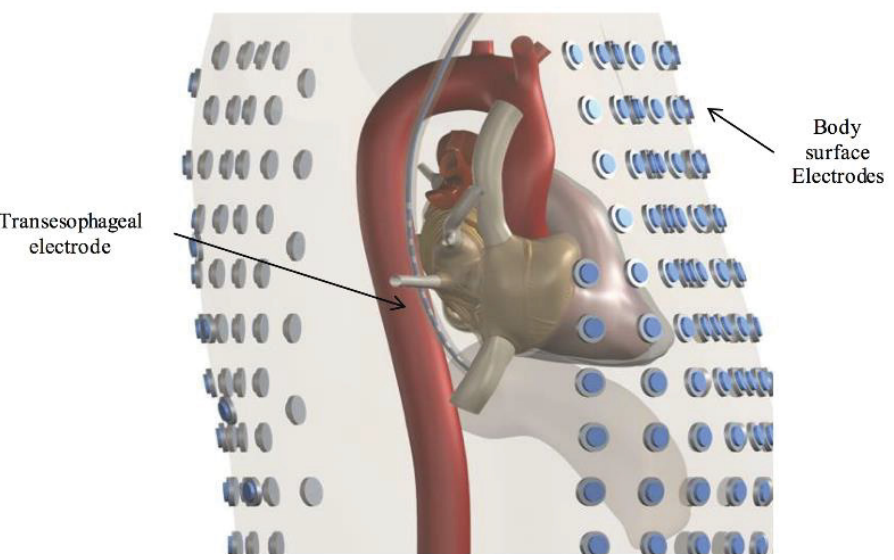

Figure 1. Anatomical model of the atria used for simulations. A) Posterior view B) Saggital view. C) Fiber orientation in anterior view. D) RA free wall fiber orientations. E) Thorax model.

\subsection{Simulation Protocol}

To exclude possible bias resulting from a particular spiral wave, five different spiral waves were initiated at different locations for each different group. To initiate each spiral wave a single pacing site in combination with an associated temporary block line was used. 


\subsection{Implementation of fibrosis}

Fibrosis segments were simulated by randomly removing transverse conductivity between elements. Three different degrees of fibrosis (slight (40\%), moderate (60\%), and severe (80\%)) were selected and simulated to be compared with no fibrosis (control).

\section{Analysis}

\subsection{Detection of Phase Singularities}

In two dimensions, the tip of a reentrant wave forms a phase singularity (PS); a point where all phases of the action potential simultaneously occurs. The three-dimensional equivalent of a PS is a vortex filament. Model elements that were part of vortex filaments were detected using the method. ${ }^{16}$ A filament was defined by the line of intersection of the two surfaces $V(r, t)=V i s o$, where $V$ iso is an arbitrary selected potential value which used to distinguish polarized and depolarized regions of the tissue (in this study $-49 \mathrm{mV})$, and $\partial_{t} V(r, t)=0$. Since in our simulations the time is discrete we could approximate the $\partial_{t} V(r, t)=0$ by $\partial_{t} V(r, t) \approx$ $V(r,(n+1) \Delta t)-V(r, n \Delta t)$. Therefore the filament position could be identified using formula (1).

$V(r, n \Delta t)=V(r,(n+1) \Delta t)=V_{\text {iso }}$

In order to describe this method more practically we represent the filament line by the set of intersection points of this line with the lattice planes defined $x=\Delta i, \mathrm{y}=\Delta j, z=k \Delta z$, which they are all the $y-z, x-z$, and $x-y$ planes that contain lattice points, respectively. Since each plane is made up of squares with one lattice point at each of the four corners, the algorithm proceeds by finding which of the $3 \mathrm{NxNyNz}$ squares contained in these planes are intersected by the line defined by Eq. (1) and what are the coordinates of these intersection. ${ }^{16}$ Finally, all of these points are sorted in order of increasing arclength $S$ along a given filament, the 
arclength distance between two nearby points $\left(p\left(p_{1}, p_{2}, p_{3}\right)\right.$ and $q\left(q_{1}, q_{2}, q_{3}\right)$ ) being simply defined by the Euclidean distance $\left(d(p, q)=\sqrt{\left(p_{1}-q_{1}\right)^{2}+\left(p_{2}-q_{2}\right)^{2}+\left(p_{3}-q_{3}\right)^{2}}\right)$.

\subsection{Detection of Fibrillation Waves}

A wave was defined as a contiguous area in which all nodes have trans-membrane voltages above the excitation threshold of $-60 \mathrm{mV}$. The number of waves was calculated each millisecond of simulated time.

\subsection{Dyssynchrony}

Dyssynchrony was defined as the percentage of nodes that were excited (membrane potential above $-60 \mathrm{mV}$ ), while the opposing segment of the atrial wall was not excited (membrane potential below $-60 \mathrm{mv}$ ).

In order to calculate dyssynchrony, model nodes were categorized either as epicardial or endocardial. Pairs of endocardial and epicardial nodes were created by finding the nearest epicardial node for each endocardial node. Dyssynchrony was defined as the number of pairs with different state (one node activated and the other not activated) divided by the total number of pairs. Dyssynchrony was computed for each millisecond of simulated time.

\subsection{Detection of Breakthroughs}

A breakthrough (BT) is a wave that appears in epicardial layer and cannot be related to the propagation of other waves in that layer. To detect BTs, wave sizes at each time step were calculated. If a wave smaller than a threshold ( 9 nodes in this study) appears within the epicardial layer and this wave has an overlap with a wave in endocardial layer, this was considered as a BT candidate. Each BT candidate was monitored and if its size increased in two folds, it was confirmed as a BT. 


\subsection{ECG reconstruction}

To construct the ECG from the simulated atrial electrical activity, we embedded the atrial model in an inhomogeneous torso model. The torso model included lungs and interactivity blood masses. Body surface potential was simulated using a bi-domain equation solved at $1-\mathrm{mm}$ resolution. ${ }^{17}$

\subsection{Statistical analysis}

All statistical analyses were performed using Graphpad software (Graphpad software Inc. 2003).

Statistical tests were performed to compare the 4 groups of simulations (control, slight, moderate, and severe fibrosis).

The average number of waves, phase singularities, breakthroughs, and dyssynchrony percentage during whole simulation period were calculated for each individual simulation. The results of the 4 groups were compared using one-way ANOVA with a Bonferroni correction.

\section{Results}

An example of a spiral wave initiation is shown in figure $2 A$. As illustrated in the figure $2 \mathrm{~B}$ the initiated spiral wave propagated and meandered on the anterior side of the LA area near the BB. In a control atrium (figure $2 \mathrm{C}$ ), the propagation pattern was relatively simple. Propagating broad fibrillation waves at the posterior LA wall between the pulmonary veins resulted in a simple pattern. In the RA free wall $V$-shaped fronts were observed around the CT and PMs due to their high conductivity in the fiber direction, but electrical dissociation between the epi- and endocardium was rare. 
A

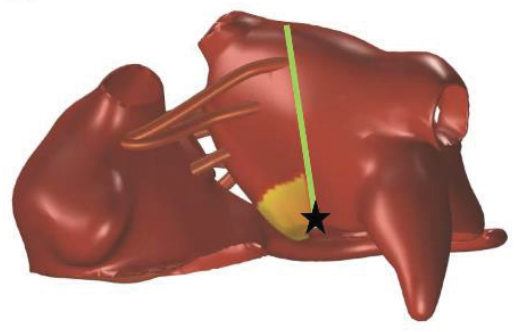

C

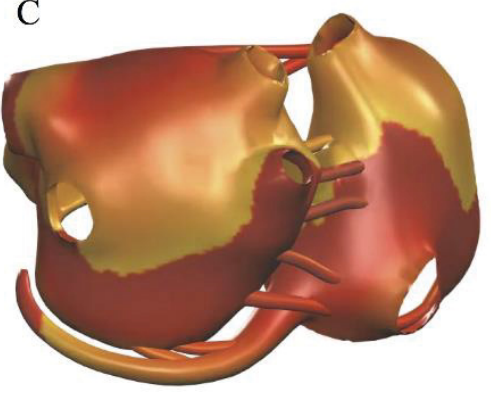

B

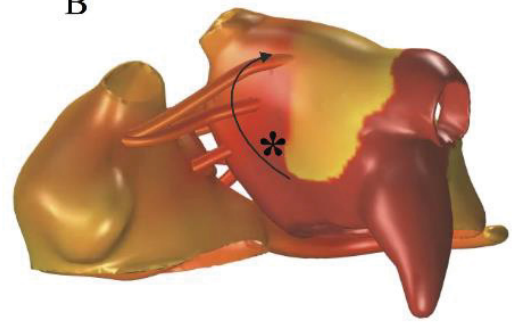

D

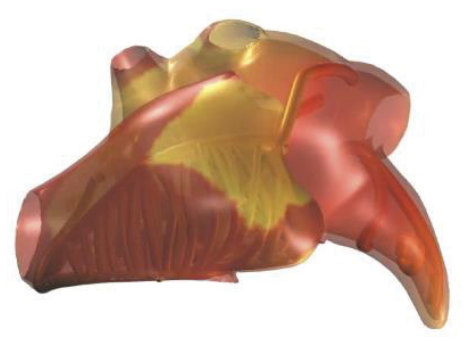

Figure 2. A) An Example of a spiral wave initiation. Pacing point (black star) and temporary line of block (green line). B) Initiated spiral wave (black asteriks) and its trajectory (black arrow). C) Potential map at LA roof and between PVs. D) Potential map at right atrial free wall.

\subsection{Effect of fibrosis}

\subsubsection{Fibrillation patterns}

In figure 3, an example of fibrillation patterns in both control and severe fibrosis are depicted. In the presence of fibrosis, fibrillation patterns in RA, LA anterior wall, and pulmonary veins area were more complex compared to control. Longitudinal dissociation, electric uncoupling of side-to-side connections between paralleloriented atrial muscle bundles, within the epicardial plane increased and waves became narrower. 


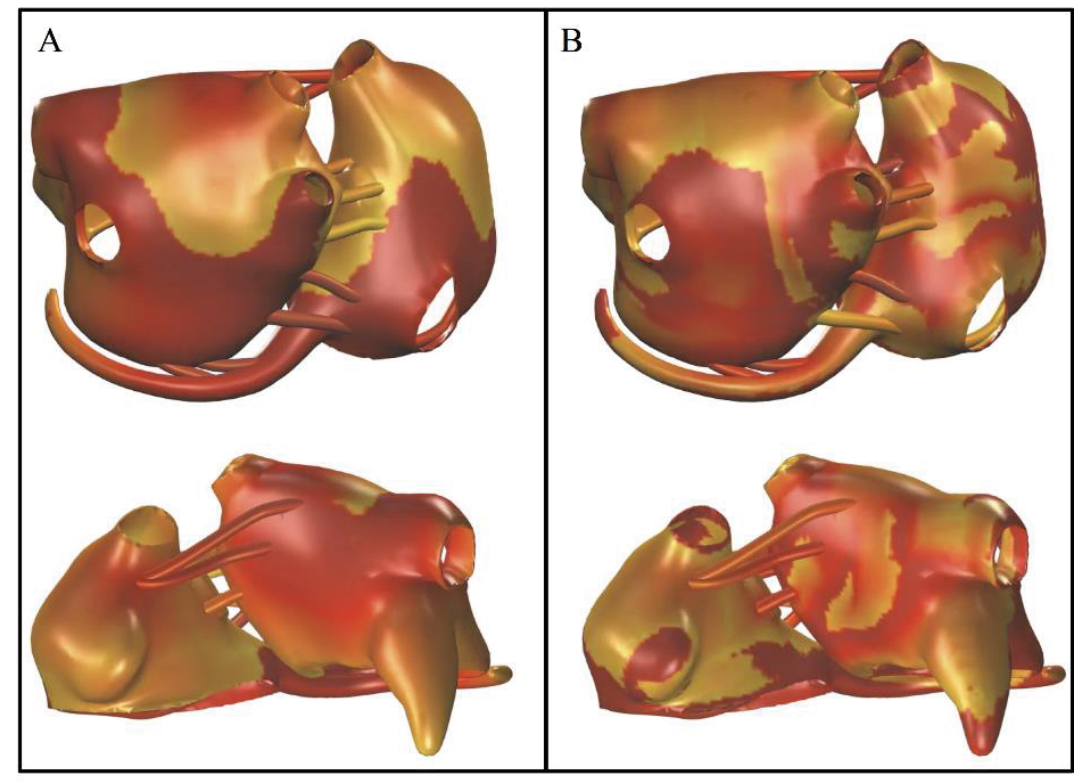

Figure 3. A) Potential map in LA and RA (control). B) Potential map in LA and RA (severe fibrosis).

\subsubsection{Dissociation and BT}

Figure 4 illustrated examples of conduction patterns in control (4A and $C$ ) and severe fibrosis (4B and D). As illustrated in this figure in both cases (control and severe fibrosis) BTs occurred but incidence of BTs in control group was more repetitive and limited to only few locations. An increase in the degree of fibrosis enhanced endo-epicardial electrical dissociation which increased the chance for BT occurrence and therefore more BTs were observed at different locations. For example, in none of the control group simulations a BTs was observed in RA free wall near the PMs or in anterior side of LA but multiple breakthroughs occurred at those sites in the presence of fibrosis (figure 4D and A). 
A

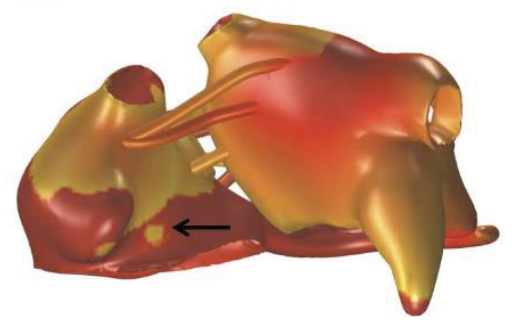

C

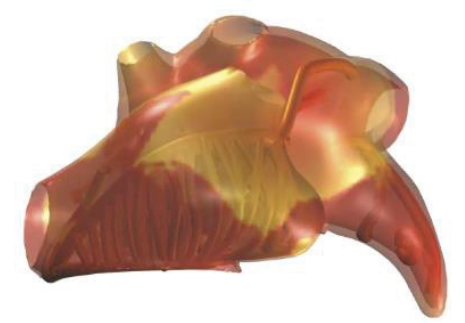

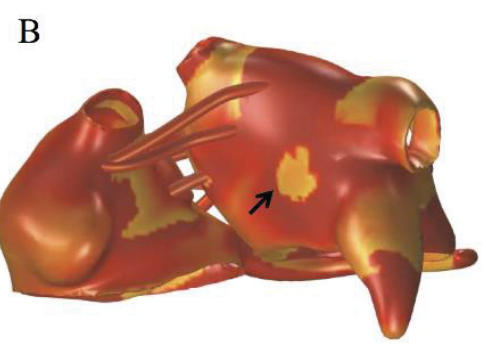

D

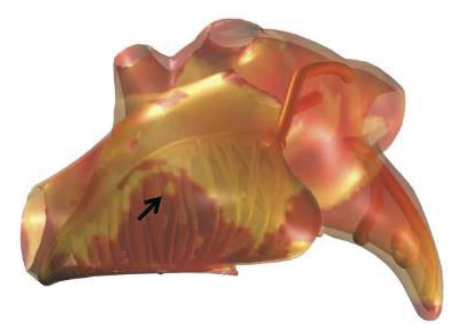

Figure 4. A) Incidence of BT in (black arrow) in control simulation. B) Incidence of BT (black arrow) in severe fibrosis simulation. C) Electrical activity association between PMs and right atrial epicardial layer. D) Increase in electrical activity dissociation in PMs and right atrial epicardial layer and incidence of BT.

\subsubsection{Quantitative analysis of fibrillation patterns}

Quantitative results extracted from 20 simulations are illustrated in figure 5. The presence of fibrosis increased the number of waves and PSs. In the case of severe fibrosis, the number of waves significantly increased compared to control and slight fibrosis. The number of phase singularities also increased significantly in the presence of severe and moderate fibrosis compared to control. Also, the number of BTs and the percentage of endo-epicardial electrical activity dyssynchrony significantly increased in the presence of severe fibrosis compared to control. 


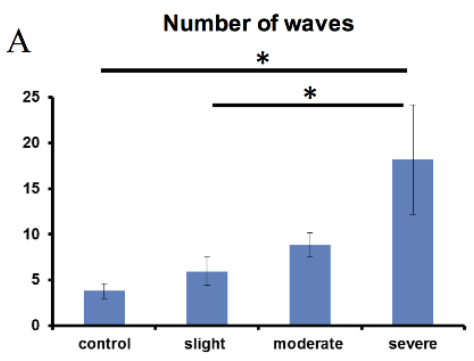

B

Number of PSs

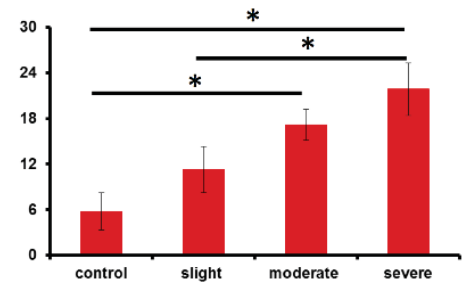

C

Dyssynchrony (\%)

D

Sum of BTs
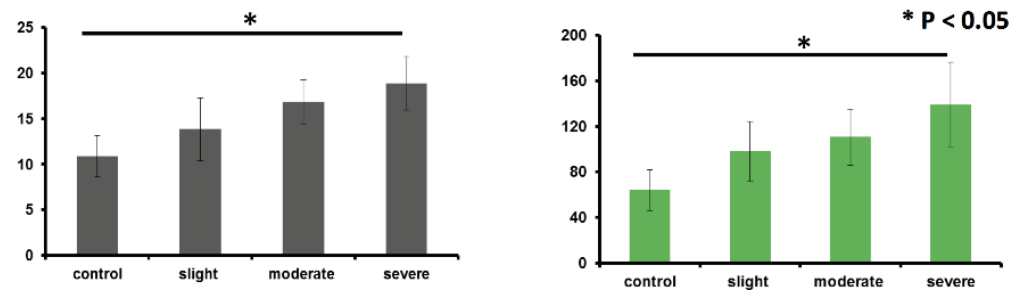

Figure 5. A) Number of waves, B) phase singularities, C) percentages of endo-epicardial electrical activity dissociation, and D) summation of all occurred BTs during simulation period in different degrees of fibrosis.

\subsubsection{Simulated ECG}

For a total of 262 body surface leads and 10 transesophageal leads, electrograms were simulated. An example of a potential map and selected ECG leads is shown in figure 6.

For all body surface and transesophageal electrodes the dominant frequency (DF) and fibrillation wave (FW) amplitudes were calculated and depicted in figure 7. The DF calculated for both projected electrograms on the body surface and transesophageal leads showed a significant reduction only in the severe fibrosis group compared to control (figure $7 \mathrm{~A}$ and $\mathrm{C}$ ). Fibrillation wave amplitudes showed a significant reduction in the moderate and severe fibrosis group compared to control, both for the surface and transesophageal electrograms. 

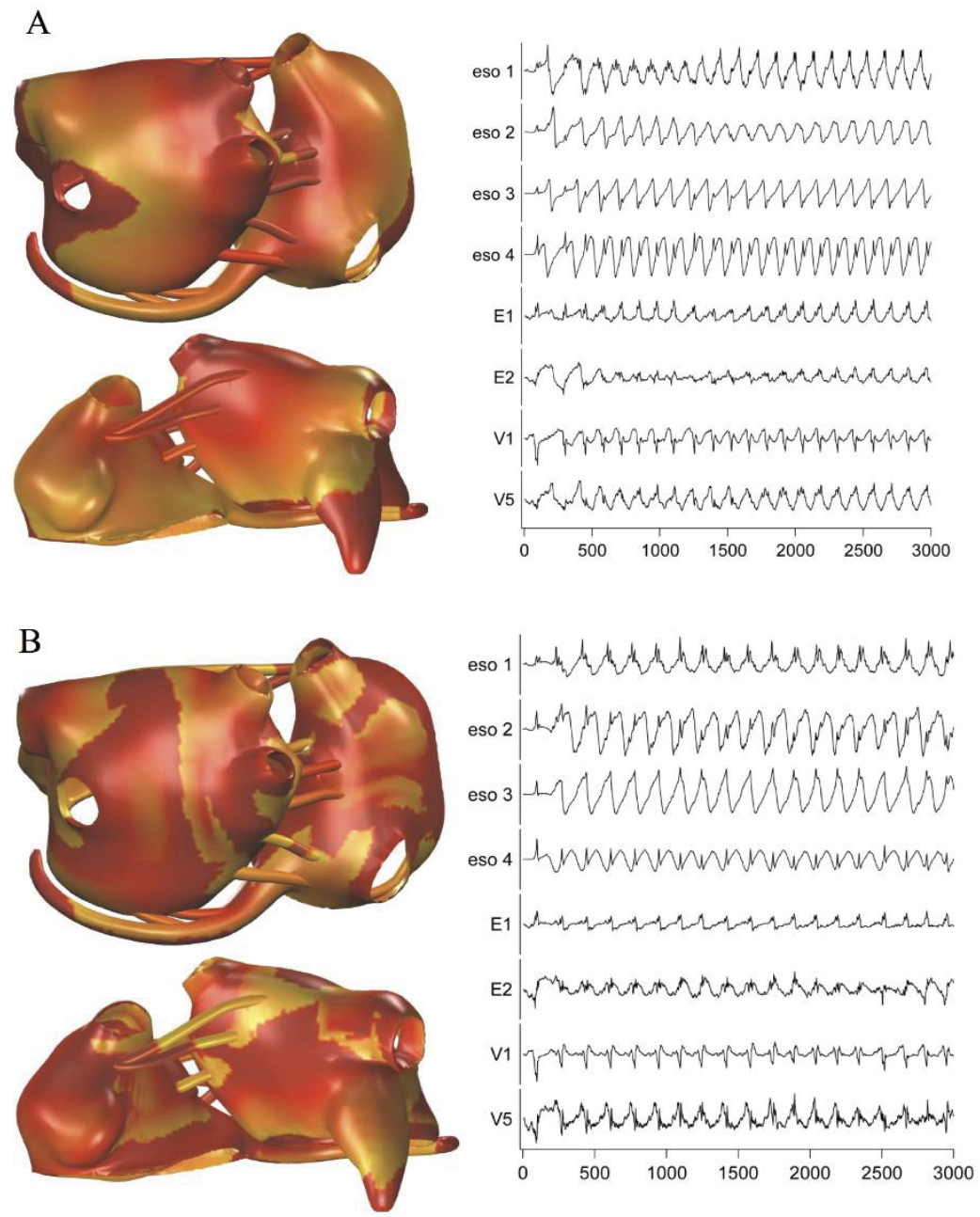

Figure 6. An example of Potential map, simulated ECGs (E1 is lead I and E2 is lead II), and transesophageal ECGs in two different groups: A) control and B) severe fibrosis. 

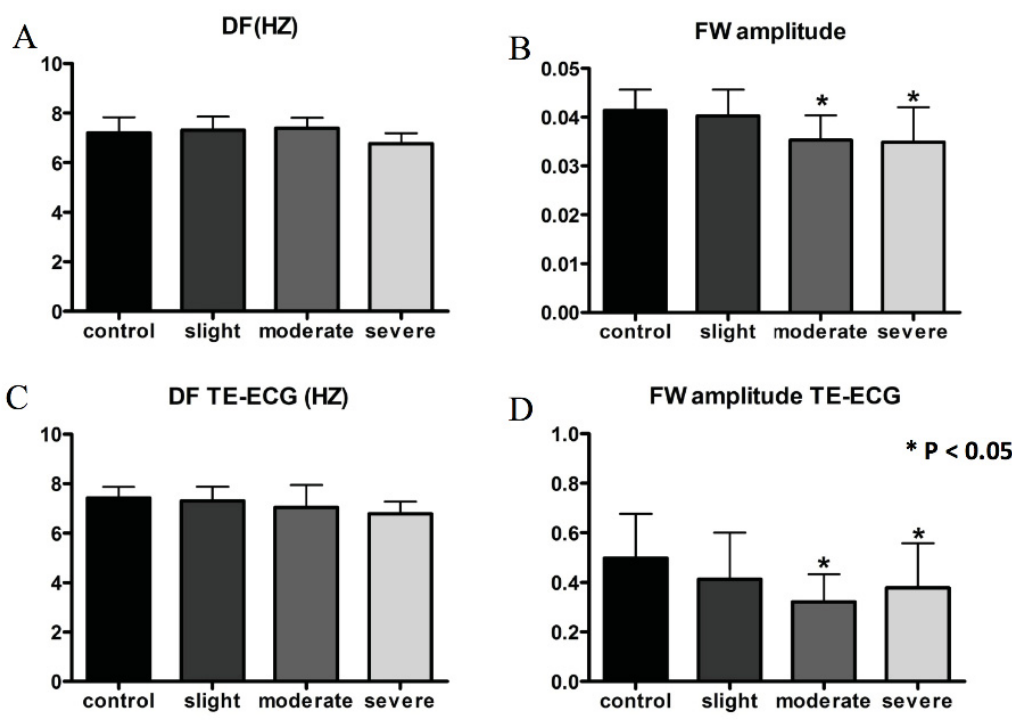

Figure 7. Calculated dominant frequencies (DF) and fibrillatory wave (FW) amplitudes. A and B) DF and FW amplitudes calculated for projected body surface potentials in the 4 different groups. $C$ and D) DF and FW amplitudes for projected transesophageal ECG (TE-ECG) in the 4 different groups.

\section{Discussion}

In this study we present a highly detailed 3D anatomical model of the human atria. This model was used to test the hypothesis that fibrosis in the epicardial layer would be sufficient to produce increased endo-epicardial dissociation of electrical activity and as a consequence an increase in transmural conduction. Our simulations demonstrate that epicardial fibrosis increased the number of waves and PSs, endo-epicardial dissociation of electrical activity and the BT rate. The higher level of AF complexity also translates into a lower DF and FW amplitudes in both transesophageal and body surface simulated electrograms. 


\subsection{Model of human atria}

We have developed a 3D human atrial model comprising realistic anatomy and fiber orientation. The global anatomy is based on MR imaging data from a human subject with normal atrial anatomy. Many anatomical details of the atrial bundle structures such as $\mathrm{PMs}, \mathrm{BB}, \mathrm{CT}$, posterior bundles connecting left and right atrium, $\mathrm{CS}$, and bundles running beneath LAA were added to this global anatomy based on a number of anatomical and histological observations. ${ }^{9,}{ }^{18}$ In order to investigate mechanisms contribute to $\mathrm{AF}$ perpetuation and stability more precisely a higher level of anatomical details such as fiber orientations should also be integrated in the model. ${ }^{10,18}$ The integrated fiber orientation in the presented model in this study was based on histological studies. ${ }^{9,10,}$

${ }^{12}$ All above mentioned properties included in this model have been observed as the most important factors determining the propagation of fibrillation waves. ${ }^{18}$ Therefore, integration of details at this high level is a necessity to investigate the endo-epicardial dissociation and transmural conduction mechanisms.

\subsection{Comparison to other Models}

Numerous modeling studies have been performed to understand the mechanisms underlying AF. Models developed for this purpose can be divided into two categories with respect to geometrical properties. The first category consists of surface models. Models in this category treat the atria as a two-dimensional sheet folded into the shape of the atria (with two main cavities, atrial appendages, and interatrial connections at the septal rings). ${ }^{19-21}$ Models in the second category are volumetric models that incorporate a greater degree of detail on the complex endocardial bundle networks and fiber orientation. ${ }^{22-27}$ Models of both categories have been used to address questions regarding $\mathrm{AF}$ mechanisms and perpetuation such as ectopy, AP heterogeneity, and fibrosis and fibrillation patterns. A few studies investigated the effect of atrial anatomy, wall thicknesses and endocardial bundles on fibrillatory patterns and AF maintenance. ${ }^{18,22,27}$ 
However, because of their design, none of these models can showed transmural conduction of fibrillation waves driven by endoepicardial dissociation of electrical activity. The novelty of our model is that it allows this phenomenon to be investigated, and to assess the effect of structural remodeling in this context. As a result of this our model is capable of simulating the occurrence of breakthroughs as a result of transmural conduction, a propagation pattern which frequently occurs during human AF. In our previous studies we highlighted the effect of endo-epicardial electrical dissociation on transmural conductions, AF pattern complexity, and AF stability using a simplified dual layer model. Chapter 2, chapter 3 , chapter 5 As a logical next step we used the realistic anatomical human atrial model to investigate the effect of endo-epicardial dissociation on transmural conduction and AF pattern complexity. To our knowledge this is the first anatomical realistic atrial model which is able to simulate the new type of fibrillation waves called 'breakthroughs'.

\subsection{Mechanisms of AF}

Several mechanisms, which contribute to AF perpetuation and progression, have been identified so far. The mechanisms underlying $\mathrm{AF}$ have complex dynamics and involve tight interactions across multiple temporal and spatial scales. ${ }^{28}$ Electrical remodeling, which occurs in the very early stages of $A F$, facilitates the progression from paroxysmal to permanent AF. This progression is also associated with irreversible structural remodeling such as elimination of myocytes side to side connections and fibrosis. ${ }^{29}$ Structural remodeling or more specifically fibrosis, which occurs in later stages of $\mathrm{AF}$, is a dominant factor stabilizing $\mathrm{AF}^{29}$

Verheule et al. showed structural remodeling in the goat AF model at later stages of AF was characterized by an increase in endomysial fibrosis in the outer layer of the atrium and would lead to a loss of side-to-side connections between muscle bundles. ${ }^{5}$ This loss can also occur between endocardial bundle networks and the epicardial layer and would lead to endo-epicardial electrical dissociation which is a prerequisite for transmural conductions. ${ }^{7,29,30}$ 
Eckstein et al. for the first time provided experimental proof for the existence of endo-epicardial dissociation in a goat model of AF and increase in endo-epicardial electrical dissociation of electrical activity over time. ${ }^{7}$ Eckstein also showed that the far majority of breakthroughs could be explained by transmural conduction while ectopic activity obviously only plays a minor role as sources of breakthrough. ${ }^{8}$ The incidence of breakthroughs not only has been observed in experimental models but also De Groot et al. showed an increase in epicardial breakthrough in patients in later stages of $\mathrm{AF}^{3}$

Based on these studies we hypothesized that endomysial fibrosis leads to dyssynchrony of electrical activation between the epicardial layer and the endocardial bundle network. This results in a $3 \mathrm{D}$ pattern of conduction in the atria which enhanced the overall complexity of AF. ${ }^{29}$

To test this hypothesis we incorporated fibrosis only in the epicardial layer by reducing transversal conductivities. Our results confirm our hypothesis and demonstrate that indeed isolated epicardial fibrosis results in an increase in number of waves, PSs, endo-epicardial electrical activity dissociation, and BTs.

\subsection{Human Torso Model}

The 3D human atrial model was incorporated into an inhomogeneous torso model which was used to simulate atrial activity electrograms, without the interference of the electrical activity of the ventricles and noises, projected on the body surface and transesophageal leads. As demonstrated in the result section increase in isolated epicardial fibrosis increased AF conduction pattern complexity and led to a decrease in both DF and FW amplitudes in projected electrograms on body surface and transesophageal leads. These findings may us to conclude that this property of this model would be very beneficial to investigate the translation of AF conduction pattern complexity in simulated body surface and transesophageal electrograms. 


\section{Study Limitations}

There were some limitations regarding this study. (1) Human experimental and histological data were sparse and limited e.g. the histological data regarding fiber orientation in the RA of the human heart is very limited. For this reason, the fiber orientation in the RA thin wall was implemented using derived histological data from goats. (2) Several studies have proposed AP heterogeneity as a main mechanism for AF initiation and perpetuation, but in this model only one myocyte model with the same characteristics was used in different region of atrium. Our model offers an excellent opportunity to investigate the presence of this property in the future. (3) Random focal discharges, which have been investigated in several $\mathrm{AF}$ computer models and can be one of the important $\mathrm{AF}$ mechanisms, are also not implemented in this model. ${ }^{25}$ 


\section{References:}

1. Schotten U, Verheule S, Kirchhof P, Goette A. Pathophysiological mechanisms of atrial fibrillation: a translational appraisal. Physiological reviews Jan 2011;91:265-325.

2. Verheule S, Tuyls E, van Hunnik A, Kuiper M, Schotten U, Allessie M. Fibrillatory conduction in the atrial free walls of goats in persistent and permanent atrial fibrillation. Circulation Arrhythmia and electrophysiology Dec 2010;3:590-599.

3. de Groot NM, Houben RP, Smeets JL, Boersma E, Schotten U, Schalij MJ, Crijns H, Allessie MA. Electropathological substrate of longstanding persistent atrial fibrillation in patients with structural heart disease: epicardial breakthrough. Circulation Oct 26 2010;122:1674-1682.

4. Allessie MA, de Groot NM, Houben RP, Schotten U, Boersma E, Smeets JL, Crijns HJ. Electropathological substrate of longstanding persistent atrial fibrillation in patients with structural heart disease: longitudinal dissociation. Circulation Arrhythmia and electrophysiology Dec 2010;3:606-615.

5. Verheule S, Tuyls E, Gharaviri A, Hulsmans S, van Hunnik A, Kuiper M, Serroyen J, Zeemering S, Kuijpers NH, Schotten U. Loss of continuity in the thin epicardial layer because of endomysial fibrosis increases the complexity of atrial fibrillatory conduction. Circulation Arrhythmia and electrophysiology Feb 2013;6:202-211.

6. Spach MS, Boineau JP. Microfibrosis produces electrical load variations due to loss of side-to-side cell connections: a major mechanism of structural heart disease arrhythmias. Pacing and clinical electrophysiology : PACE Feb 1997;20:397-413.

7. Eckstein J, Maesen B, Linz D, Zeemering S, van Hunnik A, Verheule S, Allessie M, Schotten U. Time course and mechanisms of endo-epicardial electrical dissociation during atrial fibrillation in the goat. Cardiovascular research Mar 1 2011;89:816-824.

8. Eckstein J, Zeemering S, Linz D, Maesen B, Verheule S, van Hunnik A, Crijns H, Allessie MA, Schotten U. Transmural conduction is the predominant mechanism of breakthrough during atrial fibrillation: evidence from simultaneous endo-epicardial high-density activation mapping. Circulation Arrhythmia and electrophysiology Apr 2013;6:334-341. 
9. Ho SY, Anderson RH, Sanchez-Quintana D. Atrial structure and fibres: morphologic bases of atrial conduction. Cardiovasc Res May 2002;54:325-336.

10. Ho SY, Sanchez-Quintana D. The importance of atrial structure and fibers. Clin Anat Jan 2009;22:52-63.

11. Anderson R, Becker AE. Cardiac Anatomy: An Integrated Text and Colour Atlas. Gower Medical Publishing, London 1980.

12. Maesen B, Zeemering S, Afonso C, et al. Rearrangement of atrial bundle architecture and consequent changes in anisotropy of conduction constitute the 3-dimensional substrate for atrial fibrillation. Circulation Arrhythmia and electrophysiology Oct 2013;6:967-975.

13. Potse M, Dube B, Richer J, Vinet A, Gulrajani RM. A comparison of monodomain and bidomain reaction-diffusion models for action potential propagation in the human heart. IEEE transactions on bio-medical engineering Dec 2006;53:2425-2435.

14. Krause D, Potse M, Dickopf T, Krause R, Auricchio A, Prinzen F. Hybrid Parallelization of a Large-Scale Heart Model. In: Keller R, Kramer D, Weiss J-P, eds. Facing the Multicore - Challenge II. Vol 7174: Springer Berlin Heidelberg; 2012:120-132.

15. Courtemanche M, Ramirez RJ, Nattel S. Ionic mechanisms underlying human atrial action potential properties: insights from a mathematical model. The American journal of physiology Jul 1998;275:H301-321.

16. Fenton F, Karma A. Vortex dynamics in three-dimensional continuous myocardium with fiber rotation: Filament instability and fibrillation. Chaos Mar 1998;8:20-47.

17. Potse M, Krause D, Kroon W, Murzilli R, Muzzarelli S, Regoli F, Caiani E, Prinzen FW, Krause R, Auricchio A. Patient-specific modelling of cardiac electrophysiology in heart-failure patients. Europace : European pacing, arrhythmias, and cardiac electrophysiology : journal of the working groups on cardiac pacing, arrhythmias, and cardiac cellular electrophysiology of the European Society of Cardiology Nov 2014;16 Suppl 4:iv56-iv61.

18. Tobon C, Ruiz-Villa CA, Heidenreich E, Romero L, Hornero F, Saiz J. A three-dimensional human atrial model with fiber orientation. Electrograms and arrhythmic activation patterns relationship. PloS one 2013;8:e50883.

19. Uldry L, Virag N, Lindemans F, Vesin JM, Kappenberger L. Atrial septal pacing for the termination of atrial fibrillation: study in a biophysical model of human atria. Europace : European pacing, 
arrhythmias, and cardiac electrophysiology : journal of the working groups on cardiac pacing, arrhythmias, and cardiac cellular electrophysiology of the European Society of Cardiology Nov 2012;14 Suppl 5:v112-v120.

20. Haissaguerre M, Lim KT, Jacquemet V, Rotter M, Dang L, Hocini M, Matsuo S, Knecht S, Jais P, Virag N. Atrial fibrillatory cycle length: computer simulation and potential clinical importance. Europace : European pacing, arrhythmias, and cardiac electrophysiology : journal of the working groups on cardiac pacing, arrhythmias, and cardiac cellular electrophysiology of the European Society of Cardiology Nov 2007;9 Suppl 6:vi64-70.

21. Ruchat P, Dang L, Schlaepfer J, Virag N, von Segesser LK, Kappenberger $L$. Use of a biophysical model of atrial fibrillation in the interpretation of the outcome of surgical ablation procedures. European journal of cardio-thoracic surgery : official journal of the European Association for Cardio-thoracic Surgery Jul 2007;32:90-95.

22. Aslanidi OV, Colman MA, Stott J, Dobrzynski H, Boyett MR, Holden AV, Zhang H. 3D virtual human atria: A computational platform for studying clinical atrial fibrillation. Progress in biophysics and molecular biology Oct 2011;107:156-168.

23. Colman MA, Aslanidi OV, Kharche S, Boyett MR, Garratt C, Hancox JC, Zhang H. Pro-arrhythmogenic effects of atrial fibrillation-induced electrical remodelling: insights from the three-dimensional virtual human atria. The Journal of physiology Sep 1 2013;591:4249-4272.

24. Vigmond EJ, Ruckdeschel R, Trayanova N. Reentry in a morphologically realistic atrial model. Journal of cardiovascular electrophysiology Sep 2001;12:1046-1054.

25. Gong Y, Xie F, Stein KM, Garfinkel A, Culianu CA, Lerman BB, Christini DJ. Mechanism underlying initiation of paroxysmal atrial flutter/atrial fibrillation by ectopic foci: a simulation study. Circulation Apr 24 2007;115:2094-2102.

26. Krueger MW, Severi S, Rhode K, Genovesi S, Weber FM, Vincenti A, Fabbrini P, Seemann G, Razavi R, Dossel O. Alterations of atrial electrophysiology related to hemodialysis session: insights from a multiscale computer model. Journal of electrocardiology MarApr 2011;44:176-183.

27. Krueger MW, Schulze WH, Rhode KS, Razavi R, Seemann G, Dossel $O$. Towards personalized clinical in-silico modeling of 
atrial anatomy and electrophysiology. Medical \& biological engineering \& computing Nov 2013;51:1251-1260.

28. Trayanova NA. Mathematical approaches to understanding and imaging atrial fibrillation: significance for mechanisms and management. Circulation research Apr 25 2014;114:1516-1531.

29. Verheule S, Eckstein J, Linz D, Maesen B, Bidar E, Gharaviri A, Schotten U. Role of endo-epicardial dissociation of electrical activity and transmural conduction in the development of persistent atrial fibrillation. Progress in biophysics and molecular biology Aug 2014;115:173-185.

30. Eckstein J, Schotten U. Rotors and breakthroughs as threedimensional perpetuators of atrial fibrillation. Cardiovascular research Apr 1 2012;94:8-9. 
Chapter 6 


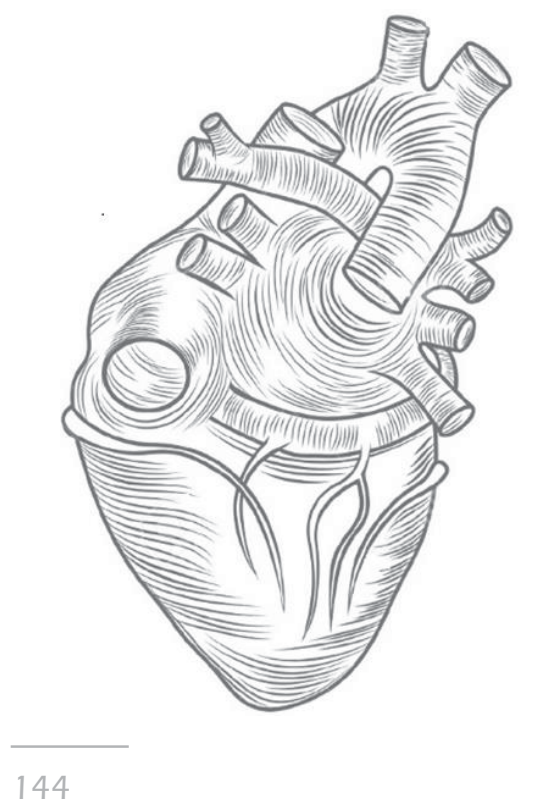




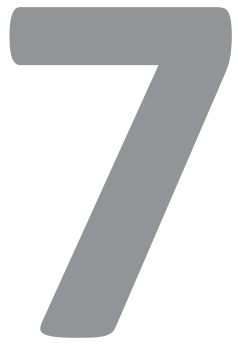

General Discussion

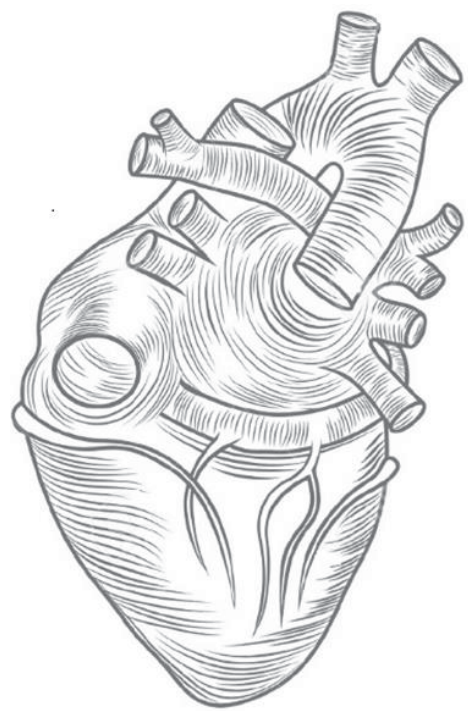




\section{Introduction}

Atrial fibrillation (AF) is the most common sustained arrhythmia and the prevalence of it increases with age. ${ }^{1}$ Therefore, the number of people suffering from AF, and the associated health care costs, are expected to rise in the coming decades with the aging of the general population. The mechanisms underlying AF have complex dynamics and involve interactions across multiple temporal and spatial scales. $^{2}$ Despite the fact that experimental electrophysiological studies on cells, tissues, organ, and whole animal or human patients have added significantly to our knowledge, the mechanisms perpetuating AF are not completely understood. ${ }^{3}$ Therefore, the efficacy of various AF treatment modalities is still unsatisfactory. Recent reviews have presented a detailed overview of our knowledge of the mechanisms contributing to initiation and progression of $A F .^{4-6}$ The progression of $A F$ involves evolution from short-lasting episodes to persistent forms of the arrhythmia. This evolution is caused by the relatively fast process of the electrical remodeling (days) and the much slower process of the structural remodeling (months to years). ${ }^{2,5}$ It is now generally accepted that paroxysmal AF is induced by the rapidly ectopic focal discharges or local reentry in the myocardial sleeves of the pulmonary veins. This hypothesis is supported by the $70-80 \%$ success rate in AF termination after only pulmonary vein isolation. ${ }^{7}$ The driving mechanisms in persistent AF is thought to involve multiple wavelet or spiral wave reentry in wide-spread regions of atria. ${ }^{8}$ This progression is also associated with irreversible structural remodeling hallmarked by the loss of side-to-side connections between myocyte bundles as a result of fibrosis. ${ }^{9}$ Structural remodeling in later stages of AF such as fibrosis is a dominant factor stabilizing AF. ${ }^{9}$ Fibrosis or fibrotic remodeling of atrial tissue comprises several parallel processes across multiple scales from membrane level to tissue level. At the tissue level, fibrotic remodeling includes both interstitial fibrosis, separating muscle bundles only within the epicardial layer but also between the epicardium and the endocardial bundle network. This would lead to a loss of electrical synchronization among the epicardial and the 
endocardial bundle network bundle. This hypothesis was investigated experimentally by a study performed by Eckstein et al. ${ }^{10}$ In that study, we demonstrated an increase in endo-epicardial dissociation of electrical activity and transmural conduction in goat AF model in 6 months of AF compared to 3 weeks of AF and acute $A F$, using in vivo high-resolution simultaneous endo-epicardial mapping.

\section{Three-dimensional Modeling of Atrial Fibrillation}

Nowadays, the rapid increase in both computational power and in our knowledge on AF mechanisms enables us to build sophisticated computer models of AF. These models can be used efficiently to study AF and mechanisms underlying its perpetuation. Therefor new hypotheses can be generated which can be late validated experimentally.

Models investigating AF, even in their most simple mathematical representation, involve propagation of atrial action potentials in a network of cells. ${ }^{2}$ The network of cells can be either arranged in a 2-dimensional form (like a simple sheet or a sheet folded into the shape of the atria) or a 3 dimensional form with a more or less detailed volumetric representation of atrial architecture. Models in the last category can be significantly different in the degree of detail included in the model. In theory, we could integrate all available scientific information in a single model but running this model would require an unrealistic amount of computational power. ${ }^{11}$

Models of AF are used to investigate the mechanisms underlying AF perpetuation should implicate electrical and structural remodeling. Electrical remodeling entails altered expression of ion channels that shorten the atrial action potential. Features of structural remodeling in experimental models of AF that can be included in models entail fibrosis, altered expression and distribution of gap junction channels and myocyte hypertrophy. ${ }^{12-14}$ Several strategies have been used to mimic fibrosis, such as (i) introducing a set of random collagenous septa disconnecting cardiac fibers in the transverse direction ${ }^{11}$ and (ii) incorporating 
non-conductive regions, with various sizes, within the tissue. ${ }^{11,13,15}$,

${ }^{16}$ In the second method the distribution of non-conductive regions could be either random or be based on imaging data. ${ }^{11,13,16}$

Fibrosis not only could affect cell to cell conductivity within the epicardial layer but also between the epicardial layer and endocardial bundle network. We propose that this loss of connectivity between these two layers explains the increase in the degree of endo-epicardial electrical dissociation and prevalence of transmural conduction (breakthroughs) that was observed experimentally. ${ }^{17}$ None of the modeling studies performed so far, investigated the mechanisms of endo-epicardial electrical dissociation and transmural propagation. In the chapter 2, we describe a novel dual layer model that enabled us to study these mechanisms and their effect on AF pattern complexity and stability. This simple dual layer was chosen because of two following reasons: (i) it is difficult to determine experimentally even with advanced imaging techniques the distribution of connections between the endocardial bundle network and epicardial layer. (ii) We aimed only to investigate quantitative determinants of endoepicardial propagation without considering the complexity of the overall 3D geometry. In this chapter we concluded that in our dual layer model AF pattern complexity and as a consequence the AF episode persistence increased compared to single layer simulations. This chapter only considered the effect of the presence of endo-epicardial dissociation and transmural conduction, due to severe loss of endo-epicardial connections because of fibrosis, on AF stability.

A more quantitative analysis of the effect of a loss of endoepicardial connections was investigated in chapter 3 . In this chapter we also studied whether the 3-dimensionality of the conduction pattern was important for AF stability. We found that all traditional electrophysiological parameters describing AF dynamics (AF cycle length, conduction velocity and action potential duration were unrelated to AF stability but that endo-epicardial electrical activity dissociation was the most important determinant of AF stability. In chapter 4, we used this model to investigate the effect of isolated epicardial endomysial fibrosis, observed in the goat model after 6 
month of AF. ${ }^{18}$ Endomysial fibrosis leads to a loss of transverse connections between myocytes in the epicardial layer. ${ }^{9}$ In the presence of endomysial fibrosis in epicardial layer only, both the number of coexisting waves and incidence of breakthrough waves in the epicardial layer significantly increased, mimicking the experimental observations in this chapter. ${ }^{18}$ This correspondence might be considered a validation of our choice of a relatively simple 'proof-of-principle' model to help explain such complex experimental observations.

The next step in the development of a 3-dimensional computer model of AF was to create a highly detailed, realistic, and anatomical model of the atrium consisting of epicardial layer and endocardial bundle network. In chapter 6 , we created such a model. It is based on various sources of anatomical information consists of a thin epicardial layer and a complex endocardial bundle network with variable degree of coupling between them. Also in this model the different degrees of epicardial fibrosis were studied. We found that an increase in endomysial fibrosis is sufficient to produce an increase in AF complexity in terms of number of waves and phase singularities and, more importantly, in endo-epicardial electrical dissociation and incidence of BTs. In addition, we modeled also a 3D thorax, which allowed us to simulate body surface potentials and transesophageal signals. Using this model we investigated the effect of alterations in the AF substrate on the surface electrograms. The comparison of recorded body surface potentials in patients at different stages of AF and simulated body surface electrograms using this model could facilitate understanding of the AF substrate in individual patients and thereby assist clinicians in diagnosis and treatment. ${ }^{2}$

The simplified dual-layer model, presented in the chapter 2 and 3 , was very helpful to understand the dynamics of transmural conduction without any confounding effect of complex 3D geometry.

In the other hand using a realistic anatomical models offers noticeable opportunities, which never could be accessible using a simplified geometry models. One of these opportunities is to answer a question regarding whether transmural conductions occur in a 
realistic anatomical atrial models and if so how it can affect conduction pattern complexities. Even we could go one step more in this regard and investigate the translation of conduction pattern complexities on the body surface potentials, which was not possible without a realistic anatomical model. It is needless to say that all achievements from highly detailed models are very expensive in terms of computational time e.g. running a realistic 3D model is 3000 times more expensive in the term of cpu-hours than a simplified geometrical model.

So far several modeling studies have been conducted to determine molecular targets for pharmacological cardioversion. ${ }^{4}$ Antiarrhythmic drugs treatment is limited due to the fact that their efficacy strongly declines with the duration of AF and their adverse side effects, such as life threatening ventricular proarrhythmia. ${ }^{2}$ Class I drugs, $\mathrm{Na}^{+}-$channel blockers, such as flecainide are used widely to treat arrhythmias. ${ }^{17}$ Despite the fact that effects of flecainide on atrial conduction and AF have been widely studied, the mechanisms by which flecainide facilitates cardioversion are still not well understood. Flecainide also loses its efficacy to cardiovert in long term AF. ${ }^{17}$ In chapter 5 , we investigated the effect of flecainide on the parameters that we showed to be the most important determinants of AF persistence in chapter 2 and 3, i.e. endo-epicardial electrical dissociation and transmural conduction. In this chapter we concluded that $\mathrm{Na}^{+}$-channel blockers could decrease AF persistence in 'healthy' atria, but that they indeed lose their efficacy with severe structural remodeling. In this chapter we tried not only to mimic clinical or experimental observations, but also investigated the possible mechanisms of the loss of efficacy in the presence of severe structural remodeling and complex AF conduction pattern. In this chapter we could demonstrate that in the presence of severe structural remodeling it is the threedimensional character of the substrate which prevents flecainide to reduce AF complexity sufficient for AF termination. 


\section{Future of computer models in the investigation of AF mechanisms}

As we demonstrated in this thesis, computer simulations of atrial electrophysiology can contribute to the understanding and interpretation of experimental data. As this development will continue there is a necessity for continued adaptation and integration of new elements, including model redesign and evaluation. Needless to say that improvement in the modeling is strongly dependent on developments of the experimental data and advances in the way we can implement imaging data in more and more realistic computer models.

Despite the unavoidable limitations of the models, we believe that this work illustrates the usefulness of computer models for the research in cardiology and we hope that these models will continue help to unravel the mechanisms underlying $A F$ and to develop better treatment for AF. 


\section{References:}

1. Wyndham CR. Atrial fibrillation: the most common arrhythmia. Texas Heart Institute journal / from the Texas Heart Institute of St Luke's Episcopal Hospital, Texas Children's Hospital 2000;27:257-267.

2. Trayanova NA. Mathematical approaches to understanding and imaging atrial fibrillation: significance for mechanisms and management. Circulation research Apr 25 2014;114:1516-1531.

3. Jalife J. Deja vu in the theories of atrial fibrillation dynamics. Cardiovascular research Mar 1 2011;89:766-775.

4. Iwasaki YK, Nishida K, Kato T, Nattel S. Atrial fibrillation pathophysiology: implications for management. Circulation Nov 15 2011;124:2264-2274.

5. Schotten U, Verheule S, Kirchhof P, Goette A. Pathophysiological mechanisms of atrial fibrillation: a translational appraisal. Physiological reviews Jan 2011;91:265-325.

6. Atienza F, Martins RP, Jalife J. Translational research in atrial fibrillation: a quest for mechanistically based diagnosis and therapy. Circulation Arrhythmia and electrophysiology Dec 2012;5:1207-1215.

7. Cappato R, Calkins H, Chen SA, et al. Updated worldwide survey on the methods, efficacy, and safety of catheter ablation for human atrial fibrillation. Circulation Arrhythmia and electrophysiology Feb 2010;3:32-38.

8. Berenfeld O, Mandapati R, Dixit S, Skanes AC, Chen J, Mansour M, Jalife J. Spatially distributed dominant excitation frequencies reveal hidden organization in atrial fibrillation in the Langendorffperfused sheep heart. Journal of cardiovascular electrophysiology Aug 2000;11:869-879.

9. Verheule S, Eckstein J, Linz D, Maesen B, Bidar E, Gharaviri A, Schotten U. Role of endo-epicardial dissociation of electrical activity and transmural conduction in the development of persistent atrial fibrillation. Progress in biophysics and molecular biology Aug 2014;115:173-185.

10. Eckstein J, Maesen B, Linz D, Zeemering S, van Hunnik A, Verheule S, Allessie M, Schotten U. Time course and mechanisms of endo-epicardial electrical dissociation during atrial fibrillation in the goat. Cardiovascular research Mar 1 2011;89:816-824. 
11. Jacquemet V, Henriquez CS. Genesis of complex fractionated atrial electrograms in zones of slow conduction: a computer model of microfibrosis. Heart rhythm : the official journal of the Heart Rhythm Society Jun 2009;6:803-810.

12. Xu J, Cui G, Esmailian F, Plunkett M, Marelli D, Ardehali A, Odim J, Laks H, Sen L. Atrial extracellular matrix remodeling and the maintenance of atrial fibrillation. Circulation Jan 27 2004;109:363-368.

13. Burstein B, Comtois P, Michael G, Nishida K, Villeneuve L, Yeh $\mathrm{YH}$, Nattel S. Changes in connexin expression and the atrial fibrillation substrate in congestive heart failure. Circulation research Dec 4 2009;105:1213-1222.

14. Nattel S, Burstein B, Dobrev D. Atrial remodeling and atrial fibrillation: mechanisms and implications. Circulation Arrhythmia and electrophysiology Apr 2008;1:62-73.

15. Comtois $\mathrm{P}, \mathrm{Nattel} \mathrm{S}$. Interactions between cardiac fibrosis spatial pattern and ionic remodeling on electrical wave propagation. Conference proceedings: Annual International Conference of the IEEE Engineering in Medicine and Biology Society IEEE Engineering in Medicine and Biology Society Annual Conference 2011;2011:4669-4672.

16. Tanaka K, Zlochiver S, Vikstrom KL, et al. Spatial distribution of fibrosis governs fibrillation wave dynamics in the posterior left atrium during heart failure. Circulation research Oct 12 2007;101:839-847.

17. Eckstein J. The Three-dimensional Substrate of Atrial Fibrillation in the Goat. Maastricht: Physiology, Maastricht; 2012.

18. Verheule S, Tuyls E, Gharaviri A, Hulsmans S, van Hunnik A, Kuiper M, Serroyen J, Zeemering S, Kuijpers NH, Schotten U. Loss of continuity in the thin epicardial layer because of endomysial fibrosis increases the complexity of atrial fibrillatory conduction. Circulation Arrhythmia and electrophysiology Feb 2013;6:202-211.

19. Eckstein J. The three-dimensional substrate of atrial fibrillation in the goat. Maastricht2012. 


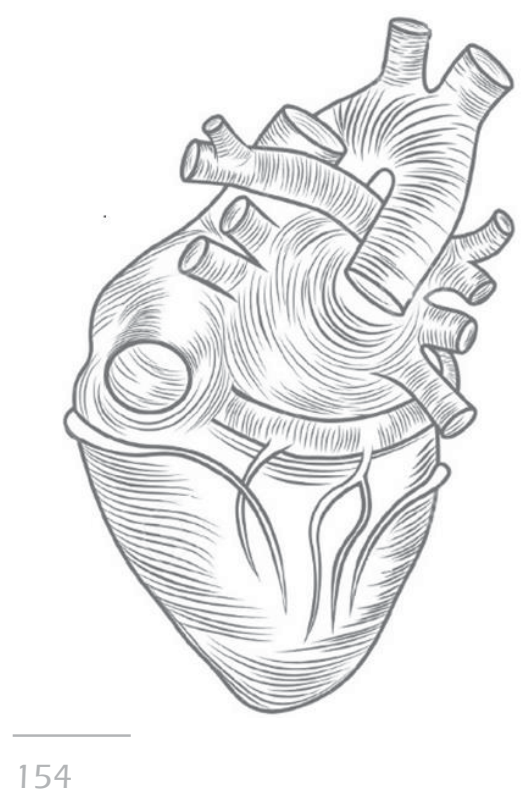




\section{Appendix}

* Supplementary Material of Chapter 3

* Supplementary Material of Chapter 4

* Summary

* Valorization

* Acknowledgements

* Curriculum Vitae

* List of Publication

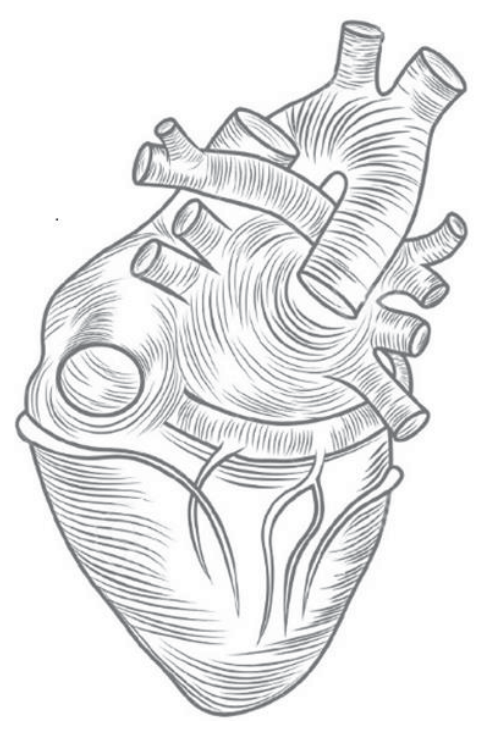




\section{Supplemental Material of Chapter 3}

\section{Supplemental methods: Algorithms Used in the Analysis section}

\section{Detection of Phase Singularities}

A phase singularity (PS), which forms the tip of a reentrant wave, was defined on the basis of a transform of membrane potential distribution into phase as described earlier. ${ }^{1}$ The algorithm used to detect PSs was based on the algorithm proposed by Zou et. al. ${ }^{2}$ Since the tip of the reentrant spiral wave is surrounded by tissue in all phases, from activation to recovery, PSs can be identified as area containing all phases of the action potential simultaneously.

\section{Computation of Lifespan of Phase Singularities}

To investigate the dynamics of PSs propagation, they were tracked in time and space as follows. The distance was calculated between all PSs detected at simulation time $t+1 \mathrm{~ms}$ and all PSs detected at simulation time $t$. If the minimum distance was less than $1 \mathrm{~mm}$, the two PSs were assigned the same ID. If for a PS the minimum distance was larger than $1 \mathrm{~mm}$, the PS was considered as an independent PS and was assigned a new ID. By choosing $1 \mathrm{~mm}$ as a threshold, it was assumed that a PS cannot move faster than $1 \mathrm{~m} / \mathrm{s}^{1}$

\section{Detection of Breakthroughs}

A breakthrough (BT) is a wave that appears in one layer and cannot be related to the propagation of other waves in the same layer. To detect BTs, areas containing connection points were studied per each $1 \mathrm{~ms}$. If a wave appeared at a connection point, this wave was followed for $2 \mathrm{~ms}$ as a candidate breakthrough. If the breakthrough candidate increased in size within $2 \mathrm{~ms}$, it was labeled as a breakthrough. The moment of breakthrough appearance and the location were stored for further analysis. 


\section{Breakthrough Propagation Tracking}

Dynamics of breakthrough propagation was explored using the wave chain tracking algorithm described in our previous work. ${ }^{1}$ In this algorithm, labeled wave(s) as well as breakthrough(s) were tracked through time and space. Lifespan of a breakthrough was defined as the time duration between breakthrough appearance and either extinction or fusion with a bigger wave. Breakthroughs were tracked in time by comparing AF patterns each $1 \mathrm{~ms}$, similar to the approach reported by Ten Tusscher et al. ${ }^{3}$ and Clayton et al. ${ }^{4}$

\section{Detection of Fibrillation Waves}

A wave was defined as a contiguous area in which all segments have trans-membrane voltages above the excitation threshold of $60 \mathrm{mV}$. Number of waves was calculated each $1 \mathrm{~ms}$ of simulation time.

\section{Computation of Wave Front Lifespan}

The propagation of fibrillation waves in our model was analyzed by tracking wave fronts through time and space. Wave fronts were detected using maximum positive slope in trans-membrane potentials.

The temporal dynamics of wave fronts can be described by three events:

- Extinction: the disappearance of a wave fronts, because it hits a boundary or runs into unexcitable tissue;

- New wave front generation: appearance of a new wave front because a wave front breaks up into two or more wave fronts;

- Fusion: the merging of two or more wave fronts into one wave front as they collide with each other.

Wave fronts were tracked in time by comparing AF wave front patterns in each $1 \mathrm{~ms}$, similar to the approach reported by Ten Tusscher et al. ${ }^{3}$ and Clayton et al. ${ }^{5}$ For all wave fronts found in each layer at simulation time $t$, overlap (amount of segments) is computed with all wave fronts found in that layer at simulation 
time $t+1$. If a wave front in one layer at simulation time $t$ does not have overlap with any wave fronts in that layer at simulation time $t+1$, it means that the wave front was extinguished. If a wave front at simulation time $t+1$ has overlap with two or more wave fronts at simulation time $t$, fusion occurred. If a wave front at simulation time $t$ has overlap with two or more wave fronts at simulation time $t+1$, wave break occurred and a new wave was generated. Finally, if a wave appeared at simulation time $t+1$ and has no overlap with any wave fronts at simulation time $t$, breakthrough occurred also resulting in a new wave front.

Identification numbers (IDs) were assigned as follows. At simulation time $t=1$, all wave fronts in both layers were detected and obtained an ID. If a wave was extinguished, the ID of that wave front was not to be used again. If, because of wave break, one or more new wave fronts were generated, the size of all new wave fronts was calculated. The largest one kept the ID of the mother wave fronts, while the remaining wave fronts obtained new IDs. If fusion occurred, the size of all wave fronts that merged was calculated and the merged wave obtained the ID of the largest one. Breakthrough wave fronts always obtained a new ID.

\section{AF Cycle Length (AFCL)}

AFCl was determined by time differences between two consecutive activation times. A square grid of $40 \times 40$ segments was used to reconstruct action potentials (APs). Using these reconstructed APs, activation time has been detected based on most positive slopes with trans-membrane potentials higher than or equal to $-60 \mathrm{mv}$.

\section{Wave Front Conduction Velocity (WFCV)}

Conduction velocity of fibrillation waves was calculated by fitting planes through each detected activation and its neighbors (grid of $15 \times 15$ electrodes), including only activations that belonged to the same fibrillation wave. The local conduction vector was calculated using the orientation of the plane and the reciprocal of the steepness of the plane. ${ }^{6}$ 


\section{Excitable Area (EA)}

Total number of segments in both layers with trans-membrane potentials below $-60 \mathrm{mv}$ is considered as amount of excitable area calculation.

\section{2-3-10. Calculation of dyssynchrony}

Dyssynchrony was defined as the percentage of segments that were excited (membrane potential above $-60 \mathrm{mV}$ ), while the opposing segment was not excited (membrane potential below $60 \mathrm{mv})$, i.e.

Dyssynchrony $=\frac{\text { number of cells activated in one layer }}{\text { total number of cells }} \times 100 \%$

Note that this formula does not take the phase of action potential (AP) into account.

To estimate the highest degree of dyssynchrony in each simulation, the average amount of dyssynchrony was calculated during the first $50 \mathrm{~ms}$ after adding the extra connection points.

\section{Endo-epicardial dissociation}

Endo-epicardial dissociation was calculated by fitting a two component Gaussian function to histograms of endo-epicardial activation time differences as described previously. ${ }^{7}$ This calculation was performed in addition to dyssynchrony to allow for direct comparison of our simulation results with previously published experimental data. ${ }^{7}$

\section{AF Survival Rate}

Percentage of simulations in which AF episodes were terminated before $6000 \mathrm{~ms}$ divided to all number of simulations in each group (8 simulations) were defined as survival rate.

\section{Numerical methods and implementation}

The model used for the present simulation study was based on our previously published bi-domain model.15 The mono-domain equation was solved assuming no-flux boundary conditions using 
an explicit numerical scheme with time steps of $0.01 \mathrm{~ms}$ as previously described.16 Gating variables and intracellular ion concentrations were updated with time steps of $0.01 \mathrm{~ms}$ during the action potential upstroke and with time steps of $0.1 \mathrm{~ms}$ otherwise. 17 Gating variables were integrated using the Rush-Larsen method.13, 18 The model was implemented in $\mathrm{C}++$ and executed on a normal PC with Intel i7 processor and 6GB memory. It took 72 hours to simulate an AF episode of $6 s$. Up to six simulations could run simultaneously on the multi-core processor without an increase in computation time.

\section{Statistical analysis}

All statistical analyses were performed using STATA 12.1 (StataCorp, 2009).

Statistical tests were performed to compare the 6 groups of simulations $(6,12,24,48,96$, and $100 \%$ connectivity), two groups of stable AF and non-stable AF, and parameters associations with AF duration.

The average number of waves, phase singularities, breakthroughs, dyssynchrony percentage, and breakthrough lifespan during whole simulation period were calculated for each individual simulation. The results of the 6 groups were compared using one-way ANOVA with a Bonferroni correction.

For comparing two groups of sustained AF and non-sustained AF, all results were tested for normal distribution. Normally distributed parameters were compared with a parametric t-test. In all other cases a non-parametric Mann-Whitney-Wilcoxon test was used.

Multilevel logistic regression analysis was used for investigation of parameter association with atrial fibrillation stability by dividing simulations in sustained and non-sustained AF. 


\section{Supplemental data:}

\section{Simulations with heterogeneous distribution of connections}

These simulations were performed as below:

1. All simulations performed in step 1 (see method section) were taken as starting point.

2. For the different numbers of connections (6, 12, 24, and 48 ), the connections were randomly distributed within one corner of the simulated tissue (quadrant $A$, see extra figure 1A).

3. All simulations in step 2 were continued either with an extra connection at the opposite corner (quadrant B, see extra figure $1 \mathrm{~A}$ ) or without the extra connection.

We did not increase the number of connections to more than 48 connections, because a higher number of connections would not fit within quadrant $A$.

As illustrated in extra figure $1 \mathrm{~B}$, we calculated the number of breakthroughs and dyssynchrony for each group. The degree of dyssynchrony was calculated in either the whole simulated tissue (total dyssynchrony) or only at the quadrant A. Dyssynchrony calculated in the corner A was strongly dependent on the number of connections and it was increased by a reduction in number of connections. Adding the extra connection in quadrant $B$ had no effect on the level of total dyssynchrony and on the dyssynchrony at quadrant A. Interestingly, a decrease in the number of connections in quadrant $A$ also had no effect on total dyssynchrony. Adding the extra connection significantly increased the occurrence of BTs. In both groups, adding or removing connections in quadrant $A$ had no significant effect on the number of BTs. Therefore, the distribution of connections is an important factor for the number of BTs modulation, and connections that are very closely apposed essentially act as a single connection. 
A)

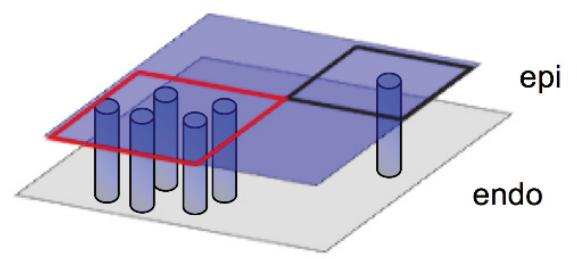

- Corner A

- Corner B

B)

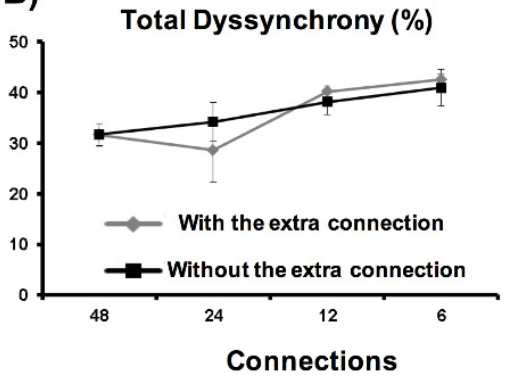

D)

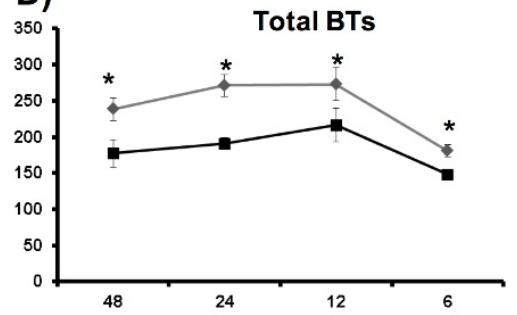

C)

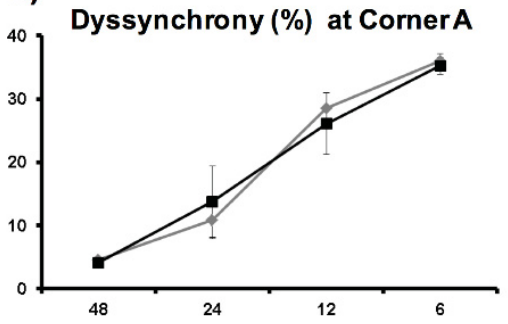

Figure S1. Heterogeneous distribution of connections. A) Model structure with a clustered distribution of connections. B) Calculated dyssynchrony in the entire simulation area. C). Calculated dyssynchrony within quadrant A. D) Total BT occurrence in simulation without and with an extra connection in quadrant $B$.

\section{Simulations with a reduced conductivity of end-epicardial connections}

Simulations with 96 connections were taken. In all segments that were located in endo-epicardial connection area, we simulated different degrees of reduction in both longitudinal and transversal conductivities. A reduction larger than $70 \%$ led to complete 
conduction block and therefore those simulations were not included in the results.

As illustrated in extra figure S2A, a decrease in conductivity increased dyssynchrony of electrical activity between the two layers. A reduction in transmural conductivity to $30 \%$ and $50 \%$ only slightly increased the dyssynchrony of electrical activity. A reduction of the transmural conductivities to $70 \%$ did have a marked impact on the electrical activity dyssynchrony.

As shown in figure S2B, the BTR did not differ significantly between the varying degrees of transmural conductivity. Interestingly, the rate of unsuccessful BTs (BTs that did not successfully propagate) in case of a $70 \%$ reduction of transmural conductivity increased dramatically. This means that with a $70 \%$ reduction in transmural conductivity, the increase in dyssynchrony did lead to more potential BTs, but that because of the reduced transmural conductivity, most of them did not propagate.

\section{A)}

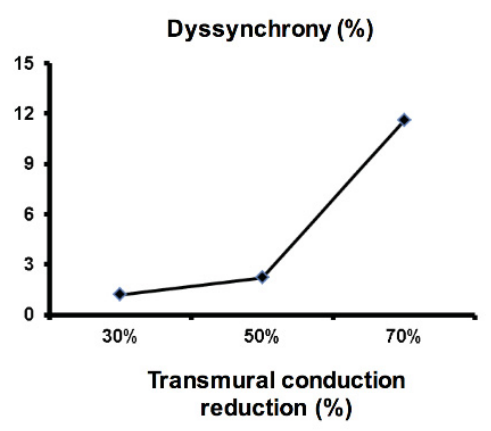

\section{B)}

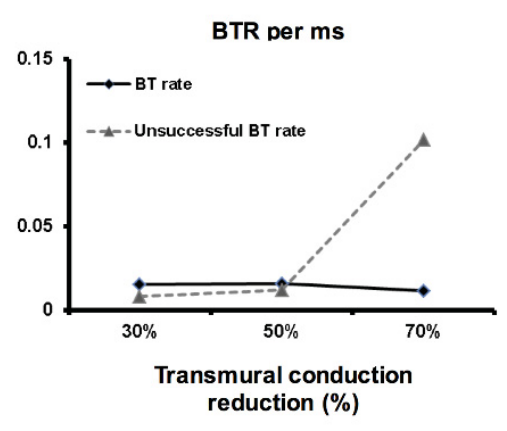

Figure S2. Reduced conductivity of end-epicardial connections. Effect of the reduction of transmural conductivity on $A$ ) degree of dyssynchrony and $B$ ) the rate of successful and unsuccessful breakthroughs. 


\section{Reference:}

1. Gharaviri A, Verheule S, Eckstein J, Potse M, Kuijpers N, Schotten U. A computer model of endo-epicardial electrical dissociation and transmural conduction during atrial fibrillation. Europace Nov 2012;14 Suppl 5:v10-v16.

2. Zou R, Kneller J, Leon LJ, Nattel S. Development of a computer algorithm for the detection of phase singularities and initial application to analyze simulations of atrial fibrillation. Chaos Sep 2002;12:764-778.

3. Ten Tusscher KH, Hren R, Panfilov AV. Organization of ventricular fibrillation in the human heart. Circ Res Jun 22 2007;100:e87-101.

4. Clayton RH, Holden AV. Dynamics and interaction of filaments in a computational model of re-entrant ventricular fibrillation. Phys Med Biol May 21 2002;47:1777-1792.

5. Clayton RH, Holden AV. A method to quantify the dynamics and complexity of re-entry in computational models of ventricular fibrillation. Phys Med Biol Jan 21 2002;47:225-238.

6. Houben RP, de Groot NM, Smeets JL, Becker AE, Lindemans FW, Allessie MA. S-wave predominance of epicardial electrograms during atrial fibrillation in humans: indirect evidence for a role of the thin subepicardial layer. Heart Rhythm Dec 2004;1:639-647.

7. Eckstein J, Maesen B, Linz D, Zeemering S, van Hunnik A, Verheule S, Allessie M, Schotten U. Time course and mechanisms of endo-epicardial electrical dissociation during atrial fibrillation in the goat. Cardiovasc Res Mar 1 2011;89:816824. 


\section{Supplemental Material of Chapter 4}

\section{Supplemental methods}

\section{Animal model}

Ten ST and 7 LT goats were included in this study (weight ST, $50 \pm 3 \mathrm{~kg}$; weight $\mathrm{LT}, 49 \pm 4 \mathrm{~kg}$ ). All animal procedures were in accordance with national and institutional guidelines. Anesthesia was induced with $10 \mathrm{mg} / \mathrm{kg}$ pentobarbital and maintained with $2 \%$ isoflurane. A bipolar screw-in J-lead (Medtronic, Minneapolis, MN) was inserted through the jugular vein into the RA and connected to an Itrel pacemaker (Medtronic) in a subcutaneous pocket in the neck. AF was maintained by applying a 1-second burst of $50 \mathrm{~Hz}$ every 2 seconds for 3 weeks (ST) or 6 months (LT).

An initial analysis of these same ST and LT goats has been published before. ${ }^{1}$ In short, we have shown that the efficacy of cardioversion with a combination of AVE0118 and dofetilide was $80 \%$ after 1 month of $\mathrm{AF}$ and $0 \%$ after 6 months of AF. Thus, the time points of sacrifice experiments in the ST and LT group reflects the stages of persistent and permanent AF, respectively. All goats had preserved cardiac output and none showed signs of heart failure.

\section{Sacrifice experiments}

After 3 weeks (ST) or 6 months (LT) of AF, an open-chest followup experiment was performed. Anesthesia was induced with thiopental $(20 \mathrm{mg} / \mathrm{kg})$ and maintained with $2 \%$ isoflurane. After leftsided thoracotomy, electrograms during $\mathrm{AF}$ were recorded from the LA and RA free walls using a round, high-density electrode array of $4 \mathrm{~cm}$ in diameter, consisting of 234 unipolar recording electrodes with an interelectrode distance of $2.4 \mathrm{~mm}$, connected to a $256-$ channel amplifier (sampling rate $1 \mathrm{kHz}$; filtering bandwidth $0.5-$ $500 \mathrm{~Hz})$. 


\section{Analysis of fibrillation patterns}

A detailed description of the analysis of fibrillation waves has been provided previously. ${ }^{1}$ Here, we have analyzed the spatial distribution of peripheral waves (i.e. waves entering the recording area from the periphery of the array), epicardial breakthroughs (i.e. waves appearing within the recording area away from the periphery), AFCLs (average interval between activations) and slow conduction (conduction times $>8 \mathrm{~ms}$ between adjacent electrodes, corresponding to a conduction velocity $\langle 30 \mathrm{~cm} / \mathrm{s}$ ). For the dispersion patterns in AFCL, all electrodes were included, for epicardial breakthroughs and block, all electrodes except for the periphery, and for peripheral waves, only electrodes at the periphery. The degree of clustering in these parameters was evaluated by calculating the Morisita Index (higher values mean more clustering) using PASSaGE spatial statistics software. ${ }^{2,3}$

\section{Optical mapping}

For optical mapping experiments, separate groups of ST $(n=8)$ and LT $(n=6)$ goats were used. After opening the chest, hearts were excised and perfused with cold cardioplegic solution. Cannulas were inserted into the coronary ostia and fixated with purse-string sutures through the aortic wall. The heart was then transferred to a tissue chamber and perfused at $37^{\circ} \mathrm{C}$ with modified KrebsHenseleit solution (concentrations in $\mathrm{mM}: \mathrm{NaCl} 118.5, \mathrm{KCl} 3$, $\mathrm{NaHCO}_{3} 25, \mathrm{KH}_{2} \mathrm{PO}_{4} 1.2, \mathrm{MgSO}_{4} 1.2$, glucose 11, $\mathrm{CaCl}_{2} 1.2$, bubbled with $95 \% \mathrm{O}_{2}$ and $5 \% \mathrm{CO}_{2}, \mathrm{pH} 7.4$ ). A bolus of the voltage-sensitive dye di-4-ANEPPS was added and filtered solution containing 10 $\mu \mathrm{M}$ of the excitation-contraction uncoupler blebbistatin was recirculated through the heart at a perfusion pressure of 100$120 \mathrm{~cm} \mathrm{H}_{2} \mathrm{O}$. An area of approximately $2 \times 2 \mathrm{~cm}$ of the LA free wall was illuminated with Luxeon-I power LEDs $(505 \mathrm{~nm}$, Philips LumiLEDs) and imaged with a 100×100 pixel Ultima-L CMOS camera (SciMedia USA Ltd.), providing a spatial resolution of approximately $200 \mu \mathrm{m}$. A unipolar stimulation electrode was placed at various sites within the field of view (range 6-12 sites per recording location, average $9 \pm 1$ ), with a reference electrode in the 
LV cavity. Imaging files were recorded at a sampling rate of $2 \mathrm{kHz}$ during pacing at an output of $1.1 \mathrm{x}$ threshold and a basic cycle length of $350 \mathrm{~ms}$. Data were analyzed offline using custom made software. For the analysis of wavefront propagation, fluorescence movies were imported in Image $\mathrm{J}$ and the wave size and circularity $\left(4 \pi *\left(\right.\right.$ area/perimeter $\left.\left.{ }^{2}\right)\right)$ was determined for each millisecond using the 'particle-analysis' function. The wavefronts were fitted with an ellipse function, and the degree and direction of anisotropy was quantified as the ratio of major to minor ellipse axis and the angle of the major axis, respectively.

After the optical recordings, the corners of the fields of view were marked and the recording location was excised, stretched to visualize the relation between the epicardial layer and endocardial bundles and photographed with back-illumination. The photographs were then transformed back to a square area with the dimensions of the field of view in the preparation using the 'puppet-warp' function in Adobe Photoshop (Adobe Systems Inc.). The epi- and endocardial bundle orientation was reconstructed from these images and projected on the recorded wavefronts to correlate wavefront propagation to bundle orientation.

\section{Histological analysis}

After the electrophysiological measurements, the complete atria were fixed in zinc-acetate buffered formalin solution (4\%, $\mathrm{pH} 5.5$ ) in 5 ST and 5 LT goats. The entire RA and LA free walls (including the appendages) were removed from the remaining atrium and embedded in Technovit-7100 plastic resin (Heraeus-Kulzer). Transverse sections of $5 \mu \mathrm{m}$ were produced in parallel to the anterior-posterior axis with a macrotome and stained with toluidine-blue in order to distinguish myocytes (dark blue) from connective tissue (light blue). For quantitative analysis, several areas of both atria were examined in the region midway between the AV ring and the interatrial septum and spanning from the anterior side of the appendage to the posterior side of free walls. Photographs were taken at low magnification (25x) to count the incidence of larger interruptions of the epicardial layer (distances 
$>0.5 \mathrm{~mm}$ devoid of myocytes in the epicardial layer) and at high magnification (200x) to determine the myocyte diameter at the level of the nucleus. In addition, thicknesses of perimysial fibrous tissue (defined as the distance between bundles of myocytes) and endomysial fibrous tissue (defined as the distance between myocytes within bundles) were determined. For each individual measurement of these parameters, the local distance to the epicardium (excluding the epicardial fibrous layer) was determined. Atrial anatomy was studied in 6 separate hearts from goats used in other experiments.

\section{Supplemental data}

\section{Supplemental figure 1: distribution of AF cycle lengths}

Methods. The average AF cycle length (AFCL) was determined for each electrode from the intervals between activation time points (maximal negative $\mathrm{dV} / \mathrm{dt}$ ). To quantify the dispersion in activation frequency, Morisita indices were calculated for the spatial distribution of the number of activations per seconds.

Results. Figure S3A shows examples of the AFCL distribution in three different ST and LT left atria, with the corresponding Morisita index in the lower right corner of each map. The maps of the LT goats show a higher degree of heterogeneity in AFCL, due to areas with a relatively long AFCL (red areas in the 1st and 3rd LT map). The Morisita index was significantly higher in LT vs. ST goats for both atria. The AFCL was not significantly different between the ST and LT group for either atrium. Connected points in figure s1B show the average AFCL for the RA and $L A$ in each individual goat included in the study. The RA had a shorter AFCL in 5 out of 10 ST goats and in 5 out of 7 LT goats. 
A

ST
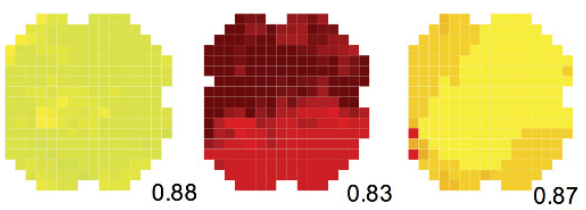

Morisita Index

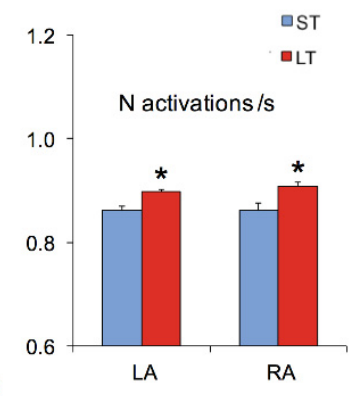

B
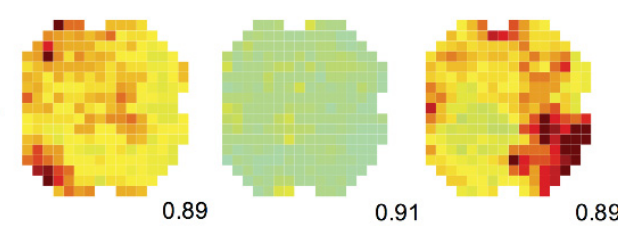

AFCL (ms) 200
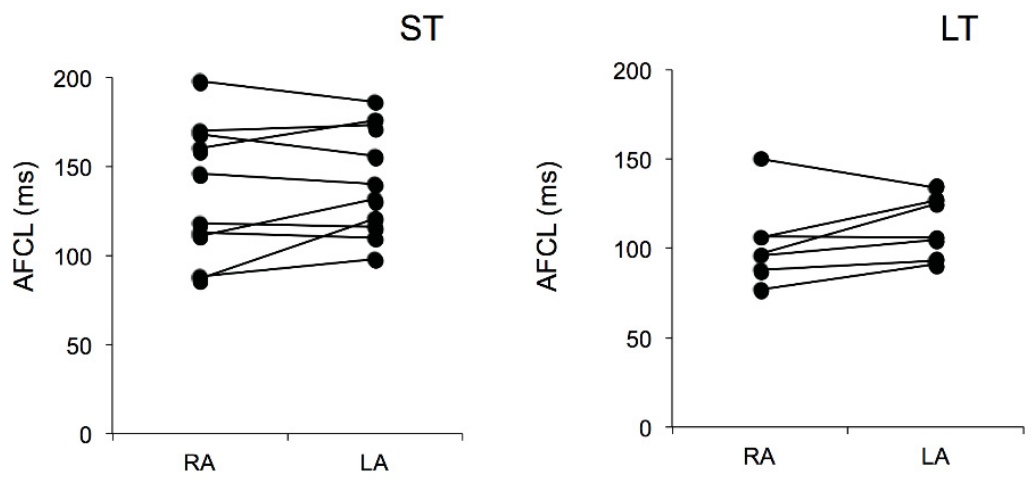

Figure S3. A) Examples of the AFCL distribution in three different ST and LT left atria, with the corresponding Morisita index in the lower right corner of each map. B) Connected points show the average AFCL for the RA and LA in each individual goat included in the study.

\section{Supplemental figure 4: overall degree of fibrosis}

Methods. To quantify the total area fraction occupied by fibrous tissue, low-magnification photos $(25 x)$ were imported in Adobe Photoshop (Adobe Systems Inc.) and the epicardial, endocardial and perivascular fibrous tissue was selected using the 'quick selection' tool and removed (figure S4A, step 1). The processed photographs were then imported in ImageJ and a threshold was set 
to distinguish fibrous tissue (light) from myocytes (dark), as illustrated in figure S4A, step 2. The area fraction of fibrous tissue was calculated as the ratio of fibrous area/ total tissue area.

Results. The total area fraction occupied by fibrous tissue was not significantly different between the ST and LT group for either atrium (figure S4B).

\section{Supplemental figure 5: determination of perimysial and endomysial fibrosis}

Methods. Figure S5 shows examples of the determination of the distribution of perimysial fibrosis (separating bundles of myocytes) and endomysial fibrosis (separating myocytes within bundles). Figure S5A shows examples for an ST and LT left atrium of the distribution of muscle bundles (outlines in red) in sections of the atrial wall stained with toluidine blue. Examples of determinations of inter-bundle distances (perimysial fibrosis) are indicated by yellow lines. Figure S5B shows an example at higher magnification of a bundle in an LT left atrium (before and after conversion to grayscale). In ImageJ, profile plots were made of the intensity across cell-to-cell boundaries, resulting in low intensity troughs for myocytes and high intensity peaks for extracellular space (ECM). The points at half-maximal intensity of each peak were taken as the transition from myocyte to ECM, in order to determine intermyocyte distances (endomysial fibrosis) and myocyte diameter.

Results. See main text. 
A
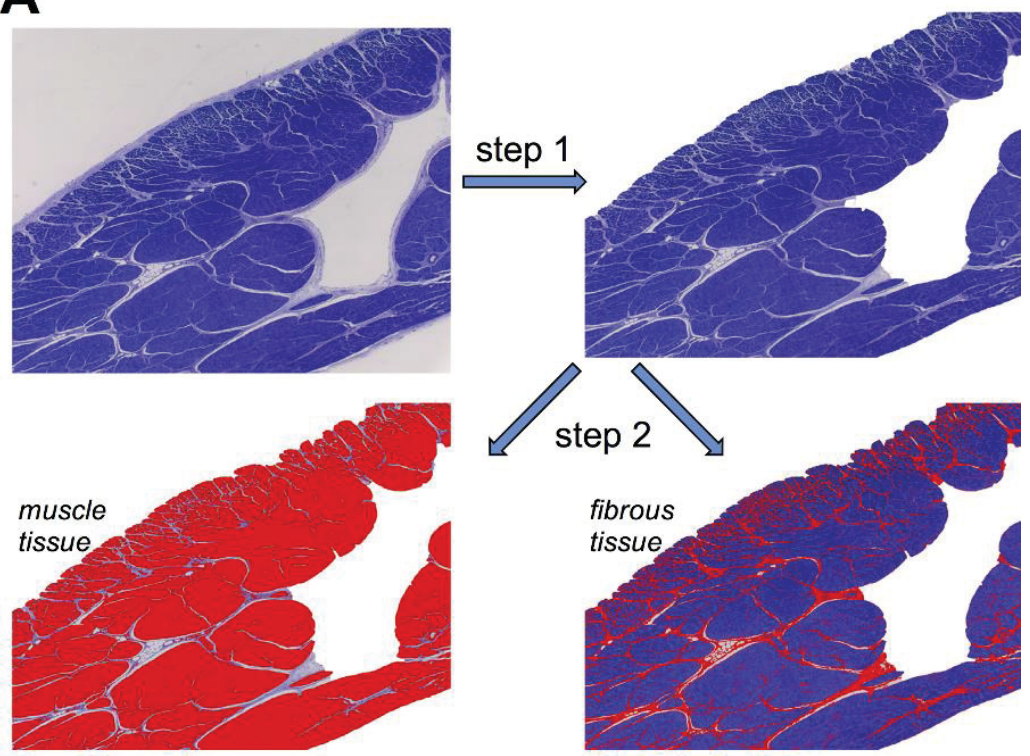

B

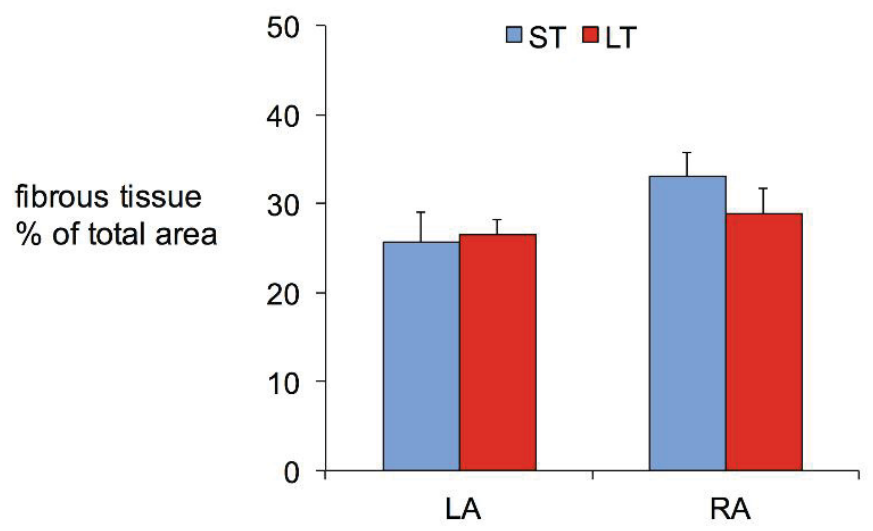

Figure S4. Removing perivascular fibrous tissue (step 1). Distinguish fibrous tissue (light) from myocytes (dark), using an arbitrary threshold (step 2). The area fraction of fibrous tissue was calculated as the ratio of fibrous area/ total tissue area. S2B) The total area fraction occupied by fibrous tissue in both the ST and LT group for either atrium. 
A
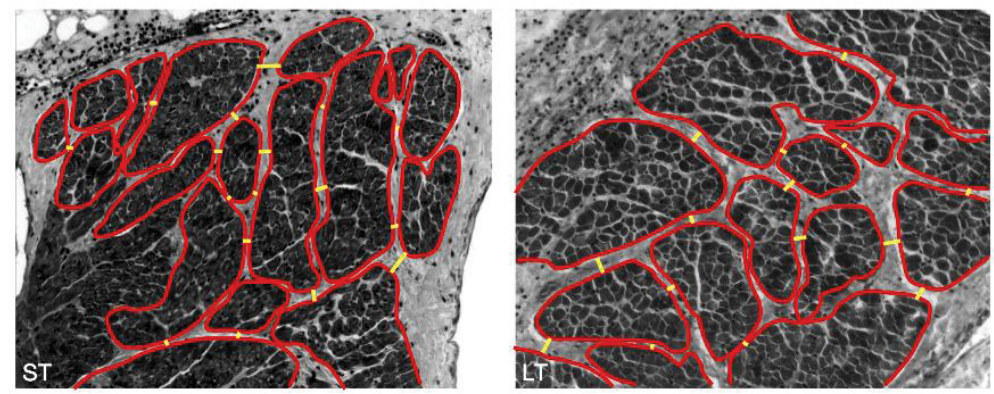

B

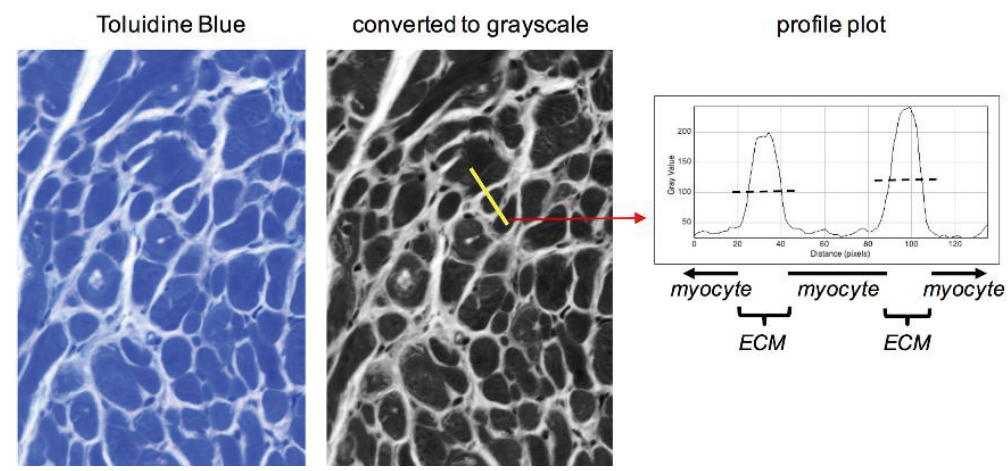

Figure S5. Examples of the determination of the distribution of perimysial. A) Examples for an ST and LT left atrium of the distribution of muscle bundles (outlines in red) in sections of the atrial wall stained with toluidine blue and examples of determinations of inter-bundle distances (perimysial fibrosis) are indicated by yellow lines. B) Example at higher magnification of a bundle in an LT left atrium (before and after conversion to grayscale). 


\section{Supplemental methods: Description of the dual-layer mathematical model}

To investigate the effect of a reduction in transverse electrical coupling in the epicardial layer on the complexity of AF and the incidence of epicardial breakthroughs, a novel proof-of-principle computer model was developed. Atrial tissue was described by a monodomain reaction-diffusion model comprising two layers of $4 \mathrm{x}$ $4 \mathrm{~cm}$. Each of the layers was composed of $400 \times 400$ segments of size $0.01 \times 0.01 \mathrm{~cm}$ and atrial membrane behavior for each segment was based on the Courtemanche-Ramirez-Nattel model. ${ }^{4}$ Electrical connections between the two layers were incorporated by adding resistances between opposing segments in a circular area with radius $0.1 \mathrm{~cm}$ (conductivity $\sigma z=0.5 \mathrm{mS} / \mathrm{cm}$ ). These connections could be introduced or removed at any time during the simulation. The monodomain model is described by

$\chi\left(C_{m} \frac{\partial V_{m}}{\partial t}+I_{\text {ion }}+I_{\text {stim }}\right)=-\nabla \cdot\left(\mathbf{G} \nabla V_{m}\right)$,

where $\chi$ is membrane surface-to-volume ratio, $C_{m}$ is membrane capacitance, $I_{i o n}$ is ionic membrane current, $I_{\text {stim }}$ is externally applied stimulus current, and $\mathbf{G}$ is the conductivity tensor. In the present study, $\chi=2000 \mathrm{~cm}^{-1}, C_{m}=1 \mu \mathrm{F} / \mathrm{cm}^{2}$. In the isotropic endocardial layer, conductivities were the same in both directions, $\sigma_{x}=\sigma_{y}=0.5 \mathrm{mS} / \mathrm{cm}$. In the anisotropic epicardial layer, $\sigma_{x}=1.0 \mathrm{mS} / \mathrm{cm}$ and $\sigma_{y}=0.12 \mathrm{mS} / \mathrm{cm}^{5}$ To simulate endomysial fibrosis, 2 out of 3 transverse connections between segments were removed at random. All simulations were performed using ionic membrane currents reflecting complete electrical remodeling, i.e. maximum conductivities for transient outward potassium current $\left(\mathrm{I}_{\mathrm{to}}\right)$ and $\mathrm{L}-$ type calcium current ( $\mathrm{I}_{\mathrm{CaL}}$ ) were reduced by 60 and $65 \%$, respectively, and maximum conductivity for inward rectifier potassium current $\left(\mathrm{I}_{\mathrm{K} 1}\right)$ was increased with $100 \%{ }^{6}$

Simulation protocol. To investigate the effect of endomysial fibrosis on fibrillatory behavior and stability of AF episodes the following simulation protocol was applied. In one of the layers, a spiral wave 
was initiated using an S1-S2 protocol, ${ }^{7}$ while the other layer was not stimulated. The situation one second after the start of the simulation was used as the starting condition for an additional 6 seconds of simulation time in which the layers were connected by 12 connections. To exclude a bias caused by a particular geometry of the connection points, 8 separate simulations were performed with different sets of 12 randomly chosen connections. Connection points for each simulation were chosen such that two connections were at least $0.2 \mathrm{~cm}$ apart. All simulations were continued for 6 more seconds, either without or with simulated endomysial fibrosis in the epicardial layer.

Analysis. AF complexity (the number of waves and epicardial breakthroughs) was analyzed for all simulations. A wave was defined as a contiguous area in which all segments have membrane potentials above the excitation threshold of $-60 \mathrm{mV}$. The number of waves was calculated for each millisecond during the entire simulation time. An epicardial breakthrough (BT), a wave that appears in the epicardial layer and cannot be linked directly to the propagation of other waves in the epicardial layer, was detected as follows. Areas containing connection points were monitored each $1 \mathrm{~ms}$. If a new wave appeared in one of these areas and could not be related to the propagation of other waves in that layer and if it had a size at most the size of the connection area, it was marked as a candidate breakthrough. If a candidate breakthrough increased in size within the next $2 \mathrm{~ms}$, it was counted as a breakthrough.

Numerical methods and implementation. The model used for the present simulation study was based on our previously published bidomain model. ${ }^{5}$ The mono-domain equation was solved assuming no-flux boundary conditions using an explicit numerical scheme with time steps of $0.01 \mathrm{~ms}$ as previously described. ${ }^{8}$ Gating variables and intracellular ion concentrations were updated with time steps of $0.01 \mathrm{~ms}$ during the action potential upstroke and otherwise with time steps of $0.1 \mathrm{~ms}^{9}{ }^{9}$ Gating variables were integrated using the Rush-Larsen method., 10 The model was implemented in $\mathrm{C}++$ and executed on a normal PC with Intel i7 
processor and 6GB memory. It took 72 hours to simulate an AF episode of $6 \mathrm{~s}$ on the double-layer model. Up to six simulations could run simultaneously on the multi-core processor without increase in computation time.

Statistical analysis. Statistical tests were performed to compare the two groups of simulations (dual-layer without fibrosis $n=8$ and dual-layer with fibrosis $n=8$ ). For each group, the average number of waves and the average number of BTs during the whole simulation time was calculated. Each data set was tested for normal distribution using the Kolmogorov-Smirnov test. An unpaired Student t-test was performed to compare normally distributed data sets. 


\section{References:}

1. Verheule S, Tuyls E, van Hunnik A, Kuiper M, Schotten U, Allessie M. Fibrillatory conduction in the atrial free walls of goats in persistent and permanent atrial fibrillation. Circulation Arrhythmia and electrophysiology. 2010;3:590-599

2. Morisita M. Measuring the dispersion and the analysis of distribution patterns. Memoires of the Faculty of Science, Kyushu University, Series E. Biology. 1959;2:215-235

3. Rosenberg MS, Anderson CD. Passage: Pattern analysis, spatial statistics and geographic exegesis. Version 2. Methods in Ecology \& Evolution. 2011;2:229-232

4. Courtemanche M, Ramirez RJ, Nattel S. Ionic mechanisms underlying human atrial action potential properties: Insights from a mathematical model. Am J Physiol. 1998;275:H301-321

5. Kuijpers NH, Keldermann RH, Arts T, Hilbers PA. Computer simulations of successful defibrillation in decoupled and nonuniform cardiac tissue. Europace. 2005;7 Suppl 2:166-177

6. Schotten U, Verheule S, Kirchhof P, Goette A. Pathophysiological mechanisms of atrial fibrillation: A translational appraisal. Physiological reviews. 2011;91:265-325

7. Leon LJ, Roberge FA, Vinet A. Simulation of two-dimensional anisotropic cardiac reentry: Effects of the wavelength on the reentry characteristics. Ann Biomed Eng. 1994;22:592-609

8. Kuijpers NH, Keldermann RH, ten Eikelder HM, Arts T, Hilbers PA. The role of the hyperpolarization-activated inward current if in arrhythmogenesis: A computer model study. IEEE Trans Biomed Eng. 2006;53:1499-1511

9. Kuijpers NH, ten Eikelder HM, Bovendeerd PH, Verheule S, Arts T, Hilbers PA. Mechanoelectric feedback leads to conduction slowing and block in acutely dilated atria: A modeling study of cardiac electromechanics. Am J Physiol Heart Circ Physiol. 2007;292:H2832-2853

10. Rush S, Larsen H. A practical algorithm for solving dynamic membrane equations. IEEE Trans Biomed Eng. 1978;25:389-392 


\section{Summary}

The study in this thesis aimed to explore the mechanism entitled "transmural conduction", which showed a significant impact on fibrillation conduction patterns in both patients in atrial fibrillation and animal models of atrial fibrillation.

In chapter 2 a novel proof-of-principle dual layer computer model was developed. Connections between these two layers could be introduced or removed anytime during the simulation period. First in only one of the layers, a spiral wave was generated, using S1-S2 protocol. After introducing the connection points between the two layers, the spiral wave degenerated into AF like conduction pattern. After 6s, connections were either retained (dual-layer simulations) or removed (single layer simulations). Dual-layer simulations, only $12 \%$ of AF episodes died out within 6 s of simulation, while $59 \%$ of AF episodes in the single layer died out. Conduction pattern complexities, indicated by the higher number of waves and phase singularities, were significantly higher in dual-layer models compared to single layer simulations. This study indicates an important role for endo-epicardial conduction for the stabilization of AF.

In chapter 3 we explored the effect of different degrees of inter layer connectivity on simulated AF episode stability and complexity. $100 \%$ connectivity served as a healthy atrium and atrial remodeling was simulated by reducing the number of connections between the layers from 96 to 6 randomly chosen connections. Progressive elimination of connections led to increase in AF stability. Moreover, reduction of the number of connections increased electrical activity dyssynchrony significantly and transmural conductions. This study was the first model illustrated the transmural conductions.

In chapter 4 we have investigated the spatial distribution of fibrillation waves and structural alterations in the atrial free walls in both a goat model of $\mathrm{AF}$ and double layer model. AF was 
maintained for 3 weeks (ST, persistent AF) or 6 months (LT, permanent AF). Fibrillation patterns were assessed with epicardial mapping. The origin of fibrillation waves and sites of conduction abnormalities were more homogeneously distributed in LT than in ST goats. Histologically, the total area fraction occupied by fibrous tissue and the degree of perimysial fibrosis (separation between myocyte bundles) were not significantly different between groups. However, endomysial fibrosis (distance between myocytes within bundles) was significantly larger in LT goats, particularly in the outer millimeter of the atria. By contrast, myocyte diameters were larger in LT goats throughout the atrial walls. High-resolution optical mapping showed that epicardial wavefront expansion was slower and more anisotropic in LT than in ST goats. Finally, a mathematical model of a simplified atrial architecture confirmed the potential impact of epicardial endomysial fibrosis on AF complexity.

In chapter 5 the efficacy loss of $\mathrm{Na}$-channel blockers in the double layer model, introduced in the early chapters in this thesis, was investigated. At various degrees of structural remodeling, we assessed the effect of $60 \%$ sodium channel block on AF stability, endo-epicardial electrical activity dissociation (EED), and complexity of fibrillatory conduction such as number of waves, phase singularities (PSs) and transmural conduction ('breakthrough', BT) in comparison with no sodium channel block (control). Na-block terminated AF in non-remodeled but not in remodeled atria. The temporal excitable gap and atrial fibrillation cycle length increased at all degrees of remodeling compared to control. Despite the increase of EED and excitable gap, $\mathrm{Na-block}$ decreased the incidence of BT because of transmural conduction block. The number of waves and phase singularities (PSs) decreased in non- remodeled atrium in the presence of sodium channel block compared to the control but remained unaltered in the structurally remodeled atrium.

In chapter 6 we have built a novel anatomical model of the atria implementing an epicardial layer with realistic fiber orientations and endocardial muscle bundles. Using the proposed model, we tested 
the hypothesis proposed by experimental findings including that increased epicardial fibrosis leads to dissociation of electrical activity between epicardial and endocardial bundle network, thus enhancing transmural conduction ("breakthrough").

Our finding showed that with no, slight, moderate, and severe fibrosis, AF complexity, quantified by the number of fibrillation waves increased significantly. Along with increasing AF complexity, EED increased by an increase in the degree of fibrosis. In the presence of fibrosis endo-epicardial conduction occurred with breakthroughs showing radial spread of activation in the epicardial layer. The number of BTs in 3 seconds of simulated AF increased significantly in the presence of severe fibrosis compared to without fibrosis.

This model is the first anatomical atrial model of AF to display BTs, a common and conspicuous feature of fibrillation patterns in experimental studies. An increase in fibrosis in the epicardial layer increased the degree of EED and the incidence of BTs, thus increasing the complexity of fibrillatory conduction. The model offers the opportunity to study transmural conduction and its contribution to AF complexity in more detail. 


\section{Valorization}

\section{AF importance and its economic burden}

Atrial fibrillation (AF) is the most common sustained arrhythmia, occurring in $1-2 \%$ of the general population. ${ }^{1}$ More than 6 million Europeans suffer from AF. The prevalence of AF is estimated to more than double in the next 50 years, as the population ages. AF confers a 5-fold risk of stroke and one in five of all strokes is attributed to this arrhythmia. The high morbidity and mortality associated with AF imposes substantial societal and healthcare cost burdens. Annual costs related to the management of AF patients in the European Union is roughly $€ 13.5$ billion. ${ }^{1}$

So far several studies have been performed to understand the underlying mechanisms perpetuating AF. However, the results from these studies are not conclusive. Better understanding of the underlying mechanisms that contribute to AF would be beneficial for AF treatment. This would lead to higher life expectancy and a reduction in therapy cost.

The work in this thesis strongly supports the importance of one of the present mechanisms during AF called "loss of transmural connectivity between endocardium and epicardial layer" and helps the scientific community to understand better this mechanism. This mechanism plays an important role in highly complex AF dynamics and is an integral part of structural remodeling. The increase in endo-epicardial electrical dissociation increases AF complexity. An increase in AF complexity increases the number of coexisting waves and thus increases AF stability.

In abstract, these finding could be helpful in both pharmaceutical interventions and ablation strategies.

\section{To whom are these research results of interest}

In-silico cardiac models were mainly designed to understand or explain better the mechanisms underlying cardiac arrhythmias or diseases. Therefore, the main audiences in this field would be only academic community or researchers in this area. However, thanks 
largely in part to the increase in computer powers, the current designed models in this field are becoming more and more sophisticated. Therefore, clinicians and biomedical companies can use the advantages of these models to test their designed algorithms or devices to detect or treat arrhythmias.

The 3D human atrial model presented in the chapter 6 of this study, is a good example of such models. The model could be used to simulate electrical activity on the anatomical realistic atrial without the interference of the ventricular electrical activity. Since this model was incorporated into an inhomogeneous torso model, the simulated atrial electrograms could be projected on the body surface and transesophageal leads. Several complexity parameters, such as dominant frequency and fibrillation wave amplitudes, could be extracted from simulated body surface electrograms in different degrees of fibrosis. These extracted complexity parameters could then be used to classify and characterize different AF conduction pattern complexities. This would be very beneficial for clinicians to detect and diagnose the AF and its stage.

\section{How useful is this research for industry?}

The presented research in this thesis is of interest for two industrial branches listed in below:

\section{- Pharmaceutical Companies}

Anti-arrhythmic drugs are still the main treatment option for AF. However, it is shown both experimentally and clinically that a drug such as Flecainide losses its efficacy in AF termination in the later stages of $\mathrm{AF}^{2}{ }^{2}$ The role of computer models in this field of research has been to uncover the mechanisms for drug action or its efficacy loss. Aspects of this thesis investigated the possible mechanisms underlying this efficacy loss. The provided simulation results in this part has provided possible mechanistic explanations regarding the lower efficacy of $\mathrm{Na}$-channel blockers treatment in patients in later stage of AF versus short term AF. These findings can be beneficial in pharmacological researches. 
We also investigated the effect of fibrosis on fibrillatory conduction pattern complexity. For the upstream therapy these findings would be beneficial if it could prevent the loss of connectivity between the epicardial layer and the endocardial bundle network, e.g. by inhibiting fibrosis.

\section{- Clinics and Biomedical companies}

Another treatment option for AF is ablations. So far several ablation strategies were introduced and used clinically. Findings in our research strongly suggest that lesions created during ablation procedure should be truly transmural. This would in fact reduce the area available to fibrillation waves and should therefore strongly reduce AF stability.

\section{How innovative is this research?}

Based on our knowledge, the presented study is novel in the atrial fibrillation modelling area. The proposed models are the first modelling studies that were able to demonstrate transmural conductions during atrial fibrillation. Additionally, we were able to calculate endo-epicardial dyssynchrony with this model. Endoepicardial dyssynchrony is a mechanism presents in later stages of AF and can have an important effect on AF stability. Using a realistic anatomical model, we also investigated the effect of transmural conduction on electrical conduction pattern complexity and its translation into body surface potentials.

\section{Road to market}

The ultimate product for cardiac modelling in the clinics should be patient specific models. A patient specific model is a tailor made computer model for each patient, based on his/her individual pathophysiological data.

Using a patient specific model, we can simulate AF for that specific patient and evaluate several therapy options specified for that patient. Most current medical diagnostic practices lead to rough 
estimates of outcomes for a particular treatment plan $^{3}$ and their outcomes usually find their basis in the results of clinical trials. However, these results might not apply directly to individual patients ${ }^{4}$ because they are based on averages. ${ }^{5}$ Patient specific modeling can be used as an alternative approval tool to tailor treatment and optimize the treatment outcome for each individual patient.

In the past decade, patient specific modelling attracted more attention from both scientific groups and governmental funding agencies around the world. This is due to the fact that it has potential to improve diagnosis and optimize clinical treatments by predicting outcomes of the therapies and surgical interventions. ${ }^{5}$ In 2006, the European Union initiated a consortium 'Structuring the Europhysiome', that led to the Virtual Physiological Human (VPH) project. ${ }^{6,7}$ This project aims to stimulate research in the field of patient specific modelling for personalized and predictive healthcare and encompasses a number of more specific subprojects. $^{8}$ In November 2007, the National Institutes of Health (NIH) posted a Funding Opportunity Announcement regarding patient specific modeling (Predictive Multiscale Models of the Physiome in Health and Disease). ${ }^{9}$ More recently, as a direct result of the American Recovery and Reinvestment Act of 2009, the National Institute of Biomedical Imaging and Bioengineering announced a challenge grant 'Towards the Virtual Patient', with the same goal as the prior $\mathrm{NIH}$ announcement.

Despite the attention, patient specific modelling still has a long way to go in order to become a standard of care in clinical practice. One possible explanation for this would be the lack of advanced technologies and mathematical algorithms in this area. Hence, many steps in the workflow from data acquisition to patient specific models still need to be performed manually. Additionally, the uncertainty in several electrophysiological parameters estimation for each patient is a huge obstacle. These parameters vary in each patient and they cannot be estimated non-invasively using current technologies. 


\section{Refrences}

1. Schotten U, Hatem S, Ravens U, et al. The European Network for Translational Research in Atrial Fibrillation (EUTRAF): objectives and initial results. Europace Oct 2015;17:1457-1466.

2. Eckstein J. The three-dimensional substrate of atrial fibrillation in the goat. Maastricht2012.

3. Ricotta JJ, Pagan J, Xenos M, Alemu Y, Einav S, Bluestein D. Cardiovascular disease management: the need for better diagnostics. Med Biol Eng Comput Nov 2008;46:1059-1068.

4. Kent DM, Hayward RA. Limitations of applying summary results of clinical trials to individual patients: the need for risk stratification. JAMA Sep 12 2007;298:1209-1212.

5. Neal ML, Kerckhoffs R. Current progress in patient-specific modeling. Brief Bioinform Jan 2010;11:111-126.

6. Hunter P, Robbins P, Noble D. The IUPS human Physiome Project. Pflugers Arch Oct 2002;445:1-9.

7. Virtual Physiological Human-Latest News. 2009; http://www.vph-noe.eu/home.

8. VPH Projects. 2009; http://vph-noe.eu/vph-projects.

9. NIH. Predictive Multiscale Models of Physiome in Health and Diseases. 2009; hrrp://grants.nih.gov/grants/guide/pafiles/PAR-08-23.html. 


\section{Acknowledgement}

This thesis represents not only my work at the keyboard, it is a milestone in more than six years of work at Maastricht University and specifically within the Physiology department. Though only my name appears on the cover of this dissertation, a great many people have contributed to its production. I owe my gratitude to all those people who have made this dissertation possible and because of whom my graduate experience has been one that I will cherish forever.

Firstly, I would like to express my deepest gratitude to my supervisor Prof. Dr. Ulrich Schotten, whose expertise, understanding, and patience, added considerably to my graduate experience. Prof. Schotten, I want to thank you again for giving me the opportunity to work at your lab and for putting up with my crazy working hours for the past six years. My experience at your lab has been nothing short of amazing. Prof. Schotten, without your motivation and encouragement I would not have considered a graduate career in my research. Dear Uli, you are the one, who truly made a difference in my life. Uli, I really appreciate your vast knowledge and skill in many areas (e.g., physiology, electro physiology, mathematics, physics, politics, and etc.). I really enjoyed our discussions in different areas.

The second person that my sincere gratitude goes to is my coadvisor, Dr. Sander Verheule. Sander, you have been always there to listen and give advices. I am deeply grateful to you for the long and inspiring discussions that helped me sort out the technical details of my work. I am also thankful to you for encouraging the use of correct grammar and consistent notation in my writings and for carefully reading and commenting on countless revisions of this manuscript.

My sincere thanks also goes to Dr. Nico Kuijpers, who provided me an opportunity to work on his computer model. Nico, you paved way of my research and helped me a lot with the problems I 
encountered. Nico, without your precious support it would be not possible to conduct this research.

I am very grateful to Dr. Potse, who offered me the opportunity to work with his fantastic computer model. Mark, thank you very much for bringing me to the INRIA in Bordeaux. It was very interesting to work with you in that institute and to learn more about the French culture. I always enjoyed our formal and informal discussions. Mark, your patience, motivation, and immense knowledge, helped me in all the time of research. Your comments and views helped me a lot to understand and enrich my ideas.

I would also like to thank my committee member, Prof. Delhaas, Prof. Dössel, Prof. Prinsen, and Dr. Virag for accepting to review my thesis.

I want to thank my paranymphs, Kelly and Arne. Arne, you were almost the first friend I made in Maastricht. Thank you very much for your hospitality and all supports you provided within these six years. I always enjoyed spending time with you. Kelly, you are a great friend with a very good sense of hummer. Every gathering with your presence was associated with fun and laughing.

Several thanks to all my fellow lab mates Hussein, Pawel, Stef, Bart, Jens, Maura, Stijn, BG, Dragan, Stephanie, and Patrick. You were all very nice and supportive.

So many thanks to all of my friends in Maastricht, who added fun and joy to my life. You guys made me feel like home in Maastricht. Nastran and Ehsan, you are very good and long-life friends of mine. Thanks for everything and all supports. Ehsan, thanks for all supports. Mehrdad, thanks for all gatherings you planned at your place and invited me. I always had great time at your place. Naser, you are a great friend. I always enjoyed spending time with you talking and listening to you singing. Maryam, you are a wonderful friend and specially a good listener and adviser. Thank you Ali, 
Behnaz, Sanaz, and Shuan for being my great and supportive friends.

Most importantly, none of this would have been possible without the love and patience of my family. My deepest gratitude goes to my lovely parents. Without your endless love, care, patience, and supports, I could never be at this position that I am now. You should know how proud I am being your son. I love you so much.

Although I never had a brother, my dear brother-like cousin, Amirreza, never made me feel this lack. Amir, you are simply amazing.

I would also thank my second family and special thanks to my mother in-law and father in-law.

Finally, I finish this acknowledgement with my lovely wife, Shayegh. Without your love, passion, and supports I could never finish my $\mathrm{PhD}$. You were always there cheering me up and stood by me through the good and bad time. I feel really blessed to have you in my life. 


\section{Curriculum Vitae}

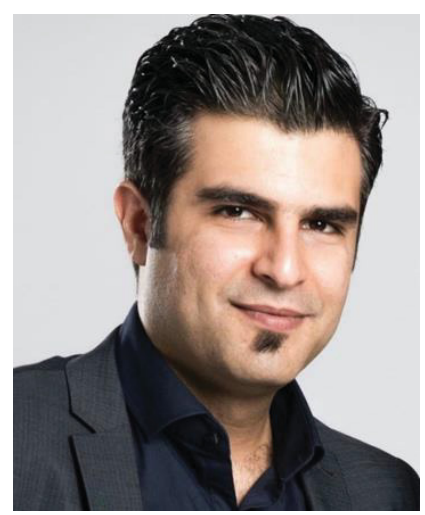

Ali Gharaviri was born on July $12^{\text {th }}$, 1982, in Tehran, Iran. After getting graduated from Alborz high school in 1999 in Tehran, he attended "Azad university, science and research branch" where he got his bachelor degree in biomedical engineering, bioelectrics. He continued his education in "Khajeh Nasir Toosi University of technology" and obtained his Master of Science degree in the field of bioelectric. In February 2009 he started working as a $\mathrm{PhD}$ student at the Physiology department of Maastricht University under supervision of Prof. Ulrich Schotten and Dr. Verheule. His research interest was focuses on computer modeling of atrial fibrillation. The PhD project was funded by Cardio Vascular Research Institute Maastricht (CARIM) and the European Network for Translational Research in AF (EUTRAF). In 2012, he achieved the Gary and Bill Sanders Poster Awards in computer in cardiology conference in Krakow. In December 2015, he moved to the Center for Computational Medicine in Cardiology (CCMC) in Lugano, in Switzerland to follow his career as a postdoc fellow in the field of in-silico modelling of atrial fibrillation. 


\section{* List of publications}

\section{Journal Articles}

Gharaviri A, Verheule S, Eckstein J, Potse M, Kuklik P, Kuijpers NH, Schotten U. How disruption of endo-epicardial electrical connections enhances endo-epicardial conduction during atrial fibrillation. Europace 2016;

Kuklik P, Bidar E, Gharaviri A, Maessen J, Schotten U. Application of phase coherence in assessment of spatial alignment of electrodes during simultaneous endocardial-epicardial direct contact mapping of atrial fibrillation. Europace 2014;16 Suppl 4:iv135-iv140.

Ganesan AN, Kuklik P, Gharaviri A, Brooks A, Chapman D, Lau DH, Roberts-Thomson KC, Sanders P. Origin and characteristics of high Shannon entropy at the pivot of locally stable rotors: insights from computational simulation. PloS one 2014;9:e110662.

Verheule S, Eckstein J, Linz D, Maesen B, Bidar E, Gharaviri A, Schotten U. Role of endo-epicardial dissociation of electrical activity and transmural conduction in the development of persistent atrial fibrillation. Progress in biophysics and molecular 2014;115:173-185.

Verheule S, Tuyls E, Gharaviri A, Hulsmans S, van Hunnik A, Kuiper M, Serroyen J, Zeemering S, Kuijpers NH, Schotten U. Loss of continuity in the thin epicardial layer because of endomysial fibrosis increases the complexity of atrial fibrillatory conduction. Circulation Arrhythmia and electrophysiology 2013;6:202-211.

Gharaviri A, Verheule S, Eckstein J, Potse M, Kuijpers NH, Schotten U. A computer model of endo-epicardial electrical dissociation and transmural conduction during atrial fibrillation. Europace 2012;14 Suppl 5:v10-v16. 


\section{In Preparation}

Gharaviri A, Eckstein J, Potse M, Verheule S, Rolf Krause, Angelo Auricchio, Schotten U. A 3D model of endo-epicardial dissociation and transmural conduction during atrial fibrillation. 
